\title{
WIRELESS IMPLANTABLE EMG SENSING MICROSYSTEM
}

\author{
by \\ BRADLEY DAVID FARNSWORTH
}

Submitted in partial fulfillment of the requirements

For the degree of Master of Science

Thesis Adviser: Professor Darrin J. Young

Department of Electrical Engineering and Computer Science

CASE WESTERN RESERVE UNIVERSITY

August, 2010 


\section{CASE WESTERN RESERVE UNIVERSITY \\ SCHOOL OF GRADUATE STUDIES}

We hereby approve the thesis/dissertation of

\section{BRADLEY DAVID FARNSWORTH}

candidate for the

Master of Science

degree *.

(signed)

Darrin J. Young

(chair of the committee)

Francis Merat

Ronald J. Triolo

(date) October 2, 2009

*We also certify that written approval has been obtained for any proprietary material contained therein. 


\section{Acknowledgment}

I would like to thank Dr. Darrin Young for his guidance throughout the duration of this project. His advice was critical to the success of this project and his attention to detail has helped me become a better engineer.

I would like to thank Dr. Ronald Triolo, whose vision for advanced prosthetics for rehabilitation motivated this project. I would also like to thank Dr. Frank Merat for serving on my thesis committee and providing helpful and insightful comments.

This project has been supported by the Advanced Platform Technology Center at the Cleveland VA Medical Center. It would not have been possible without the technical assistance of Brad Boggs and administrative support from Suzana Iveljic. I would also like to thank Dr. Dawn Taylor and Michael Stetner who facilitated our rat implant study. Thank you also to Dr. Christian Zorman and Jeremy Dunning and who designed and fabricated the prototype flexible EMG electrode substrate for this project.

Many thanks are due to all the members within my research group, especially Peng Cong, Nattapon Chaimanonart, Mark Zimmerman, and Michael Suster. They are great friends and make a great team.

Finally, I would like to thank my family for their unlimited support and encouragement. 


\section{Table of Contents}

Chapter 1 Introduction............................................................................................................... 8

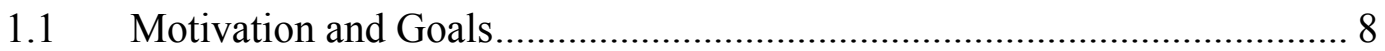

1.2 Thesis Organization ......................................................................... 12

Chapter 2 Wireless Implantable EMG Sensor for Myoelectrically-Controlled

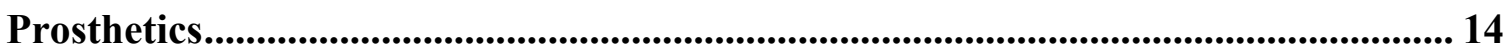

2.1 Overview of Wireless EMG-Sensing Application.................................... 14

2.2 Design Requirements .......................................................................... 16

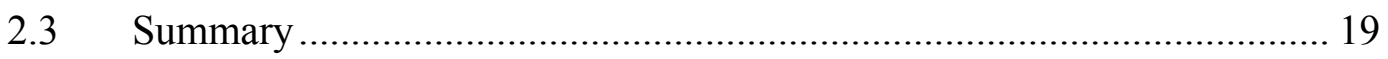

Chapter 3 Wireless Implantable EMG Sensing Microsystem Overview.................... 20

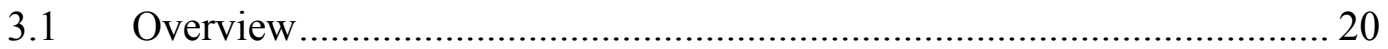

3.2 Low-Power and Low-Noise Front-End Amplifier.................................... 21

3.3 11-bit Differential Cyclic ADC …………………................................ 23

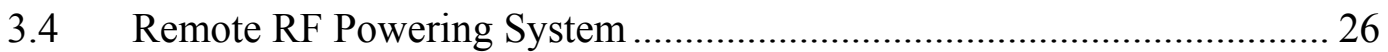

3.5 Phase-Shift Keying Passive Data Telemetry System............................... 28

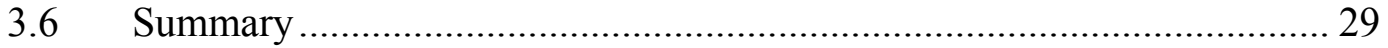

Chapter 4 Integrated CMOS EMG Sensing Electronics................................................ 31

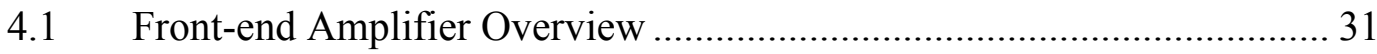

4.2 Differential Operational Transconductance Amplifier Design.................. 32

4.3 Continuous-Time Output Common-Mode Feedback Circuit and Amplifier

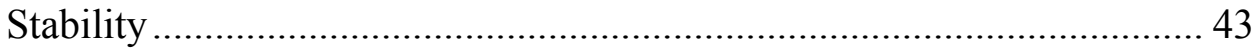

4.4 Output Common-Mode Feedback Redesign.............................................. 50

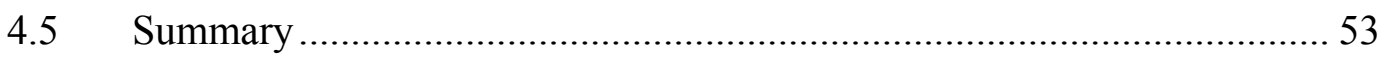

Chapter 5 Remote RF Powering and Data Telemetry System ....................................... 55

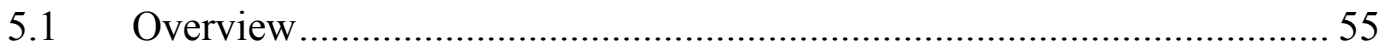

5.2 Analysis of Inductively Coupled Circuit ................................................. 56

5.3 Design Optimization of RF Power Coils .................................................... 59

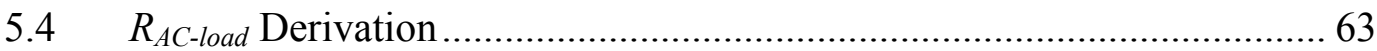

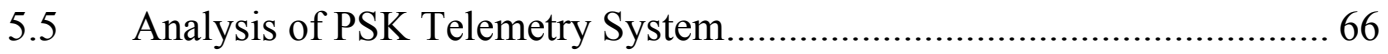

$5.6 \quad 60 \mathrm{~Hz}$ Attenuation Investigation .......................................................... 70

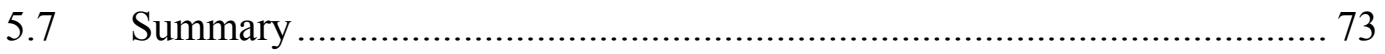


Chapter 6 Measurement Results .................................................................................... 75

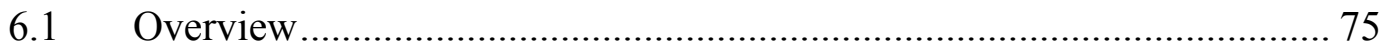

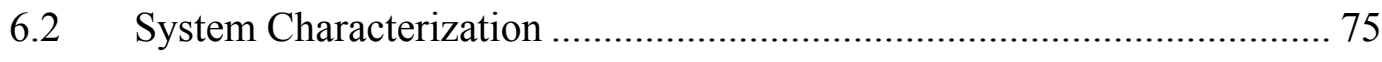

6.3 In Vivo Animal Test Results .................................................................. 92

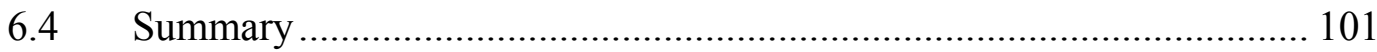

Chapter 7 Conclusion and Future Work .......................................................................... 103

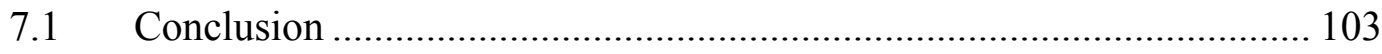

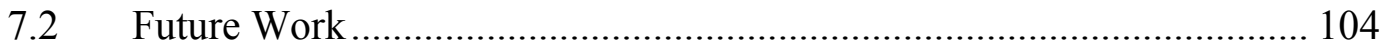

Appendix I Correlation Alignment Algorithm............................................................... 109

References........................................................................................................................... 112 


\section{List of Figures}

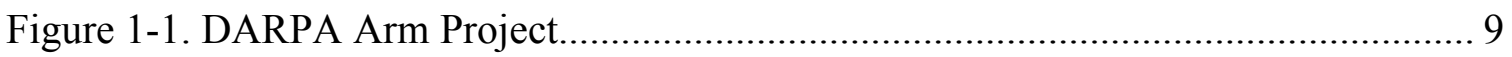

Figure 1-2. President Bush Jogging with Amputee Soldier............................................. 10

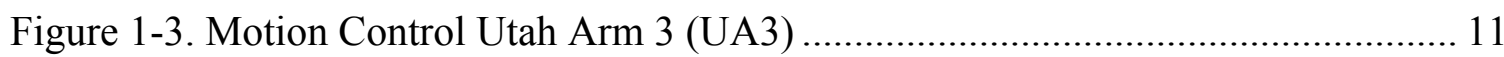

Figure 2-1. BION1 Package for Functional Electrical Stimulation................................. 15

Figure 2-2. Wireless EMG Prosthesis Control Application............................................ 16

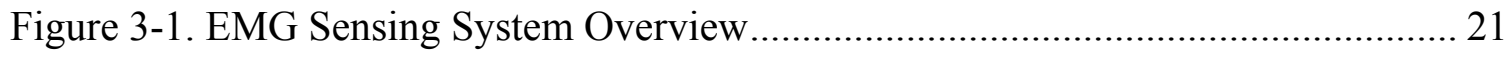

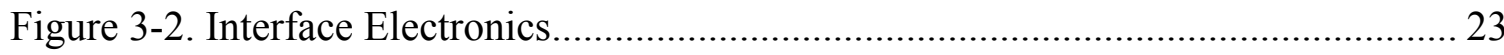

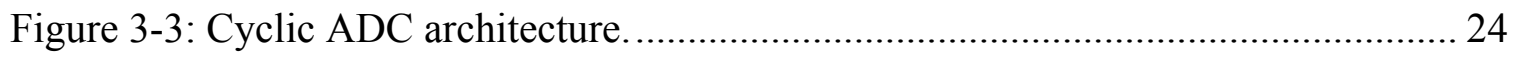

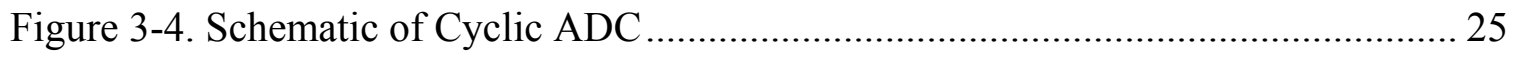

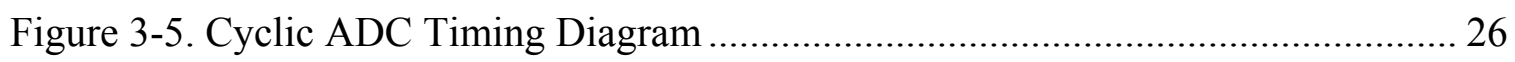

Figure 3-6. Wireless Powering Displacement Sensitivity .............................................. 28

Figure 3-7. RF Powering and PSK Data Telemetry System........................................... 29

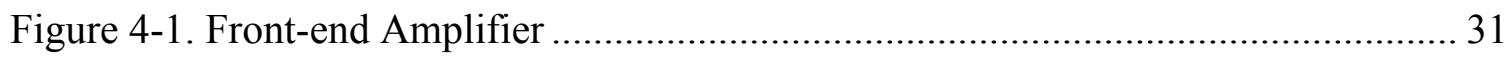

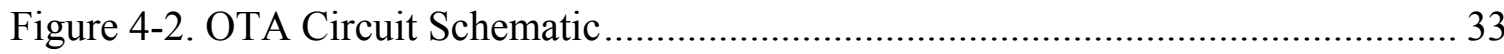

Figure 4-3. Small-Signal Transconductance vs. Inversion Coefficient ............................. 37

Figure 4-4. Open-Loop OTA Frequency Response Simulation ...................................... 42

Figure 4-5. Closed-Loop OTA Frequency Response Simulation...................................... 43

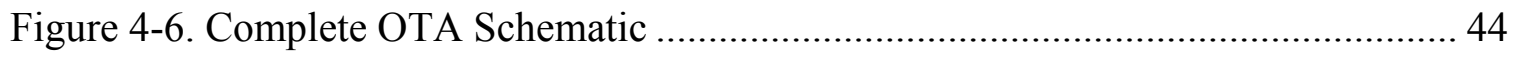

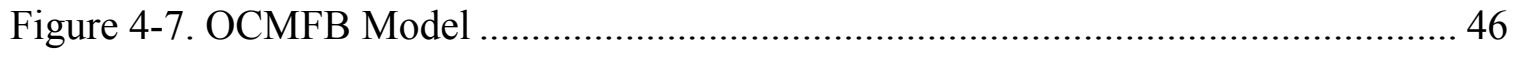

Figure 4-8. Open-Loop OCMFB 2-Stage HSPICE Simulation Result.............................. 50

Figure 4-9. Redesigned OCMFB Frequency Response ……………………………....... 53

Figure 5-1. Simplified RF Powering Schematic ........................................................ 57 
Figure 5-2. Class-E Amplifier for RF Powering..................................................... 58

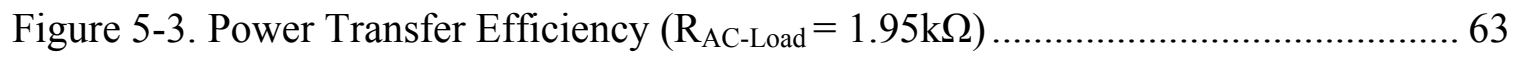

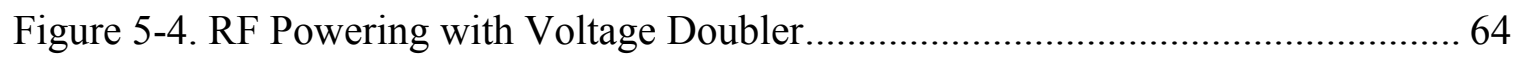

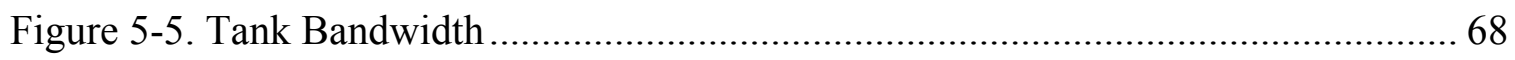

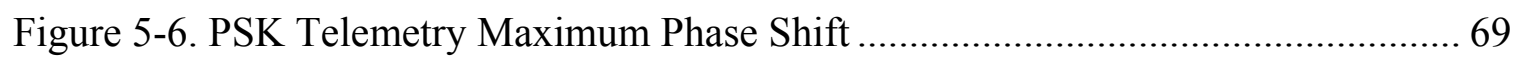

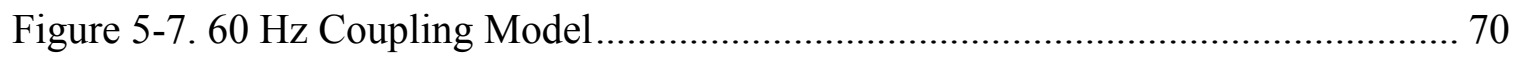

Figure 5-8. Noise Floor vs. Termination Resistor Value ........................................ 72

Figure 5-9. Closed-Loop Signal Gain vs. Termination Resistor Value ........................... 73

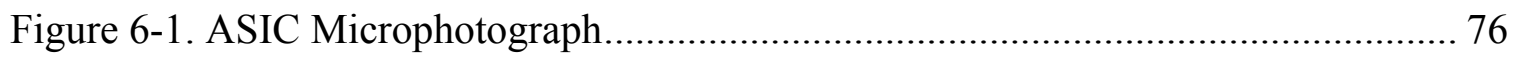

Figure 6-2. EMG Amplifier Frequency Response ................................................ 77

Figure 6-3. EMG Amplifier Output Thermal Noise Floor .......................................... 78

Figure 6-4. EMG Amplifier 1/f Noise Corner Frequency.......................................... 78

Figure 6-5. ADC Output Spectrum with DC Battery ….......................................... 79

Figure 6-6. Measured Effective Number of Bits .................................................. 82

Figure 6-7. System ENOB Effect due to Powering Schemes ...................................... 83

Figure 6-8. ADC Output Spectrum with RF Powering........................................... 83

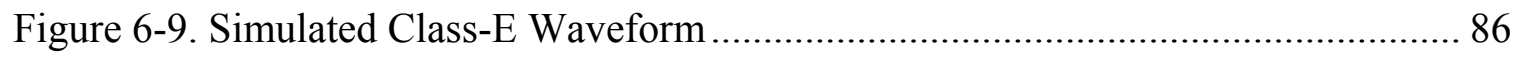

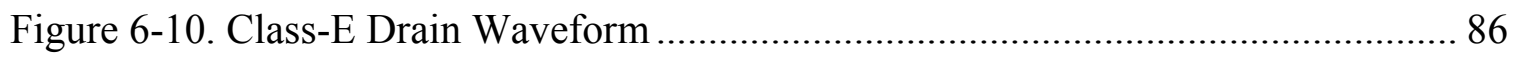

Figure 6-11. Class-E Drain Voltage vs. DC Supply Voltage .................................... 87

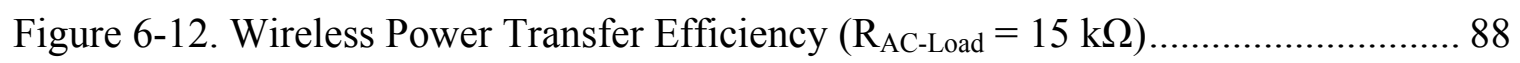

Figure 6-13. EMG Microsystem Power Consumption Breakdown.............................. 89

Figure 6-14. Transmitted and Received PSK Data ................................................ 91

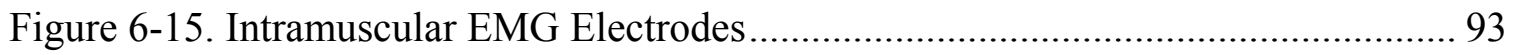




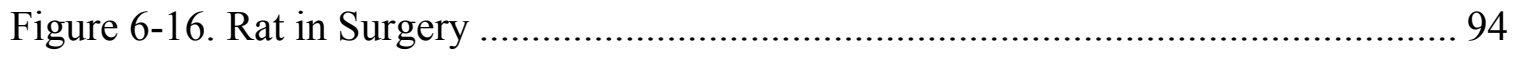

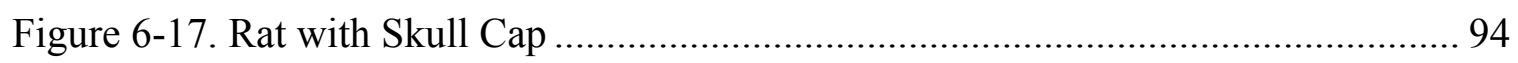

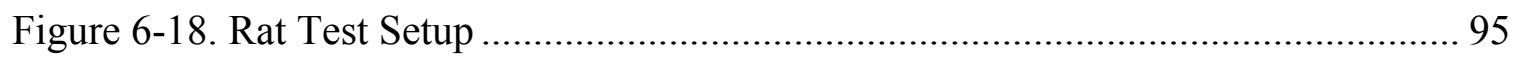

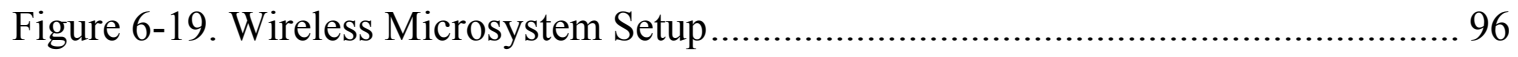

Figure 6-20. Measurement with Shorted Long Wires .................................................... 97

Figure 6-21. Long Wires Connected to Intramuscular Electrodes, with Resting Animal 97

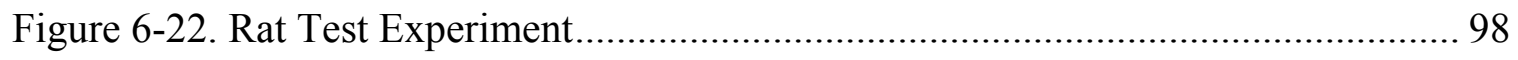

Figure 6-23. Three Rat Contractions in Four Seconds ………………………….......... 99

Figure 6-24. Three Quick Rat Hind Limb Contractions ..................................................... 99

Figure 6-25. Two Sets of Two Quick Rat Leg Contractions .......................................... 100

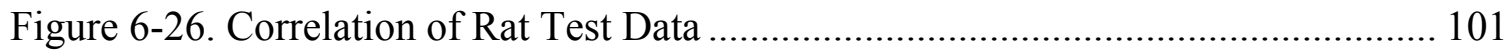

Figure 7-1. Mirofabricated EMG Electrode Prototype ................................................... 105

Figure 7-2. Ardiem flexible EMG prototype ………………................................... 106

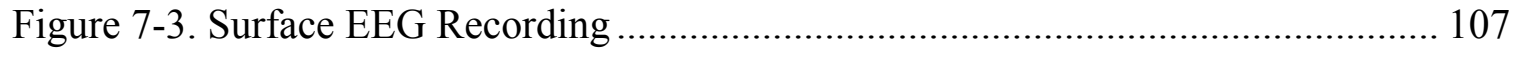

Figure 7-4. ECoG Implant and Post-Op X-Ray .......................................................... 108 


\section{List of Tables}

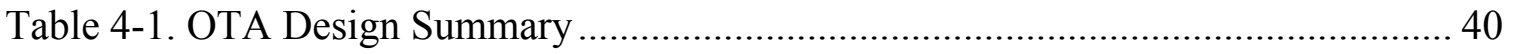

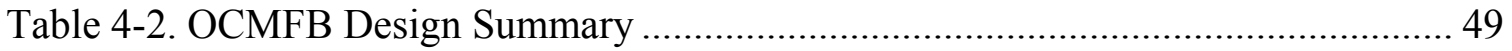

Table 4-3. Revised OCMFB Design..................................................................... 52

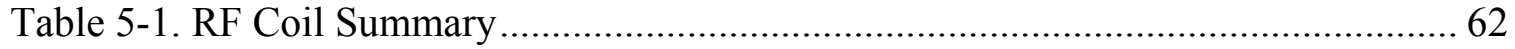

Table 6-1. Class-E Amplifier Parameters ................................................................... 85 


\section{Wireless Implantable EMG Sensing Microsystem}

Abstract

by

\section{BRADLEY DAVID FARNSWORTH}

An implantable wireless electromyogram sensing microsystem is developed for control of powered prostheses. RF powering and wireless data link enable real-time wireless operation. EMG signals are interfaced with a capacitively-coupled differential amplifier with $1 \mathrm{kHz}$ bandwidth and an input-referred voltage noise power spectral density of $78 \mathrm{nV} / \sqrt{\mathrm{Hz}}$. The EMG signal is digitized by an on-chip 11-bit cyclic ADC. A Class-E amplifier operating at $8 \mathrm{MHz}$ drives a 2-cm spiral coil, inductively coupled to an implantable 6-mm spiral coil. RF energy is rectified and regulated on-chip to produce stable $2.1 \mathrm{~V}$ and $2.7 \mathrm{~V}$ supplies. Manchester-encoded EMG data are transmitted by PSK

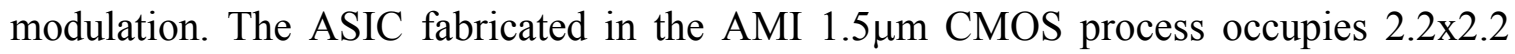
$\mathrm{mm}^{2}$ and dissipates $350 \mu \mathrm{W}$ from RF powering. The system achieves 9.35 ENOB under battery powering and 8.6 ENOB under RF power, equivalent to an input detectable signal of $5.4 \mu \mathrm{V}_{\mathrm{RMS}}$ and $9 \mu \mathrm{V}_{\mathrm{RMS}}$, respectively. In vivo testing shows a background EMG signal of $20 \mu \mathrm{V}_{\mathrm{RMS}}$, limiting system sensing resolution. 


\section{Chapter 1}

\section{Introduction}

\subsection{Motivation and Goals}

Recently there have been a great deal of publicity and research interest in intelligent powered prostheses that utilize neural interfaces for natural control. The DARPA Revolutionizing Prosthetics, or "DARPA Arm" project [1] has advanced the state-of-theart in upper extremity prosthetics by developing a complete artificial upper limb that responds directly to neural control. Residual nerves that previously controlled an amputee's arm muscles are reattached to innervate muscles in the pectoral region proximal to the injury. These newly targeted pectoral muscles contract when a user thinks about moving muscles in his missing limb. These contractions are monitored and interpreted as prosthesis control signals.

In the 2007 version of the DARPA arm, surface-recording EMG electrodes were attached to pick up this neural control signal. While this was a successful approach, there are several critical issues associated with surface EMG recording that warrant attention. Most importantly, surface EMG electrodes must be attached to the appropriate recording sites by the prosthesis user daily. This requires preparation of skin surface, proper positioning of the recording electrodes, and careful routing of the electrode lead cables to the prosthesis electronics. Surface electrodes are prone to motion artifacts due to lead 
movement as well as poor signal quality resulting from skin perspiration or poor placement technique or location. A patient wearing the surface electrode version of the DARPA Arm is shown in Figure 1-1.

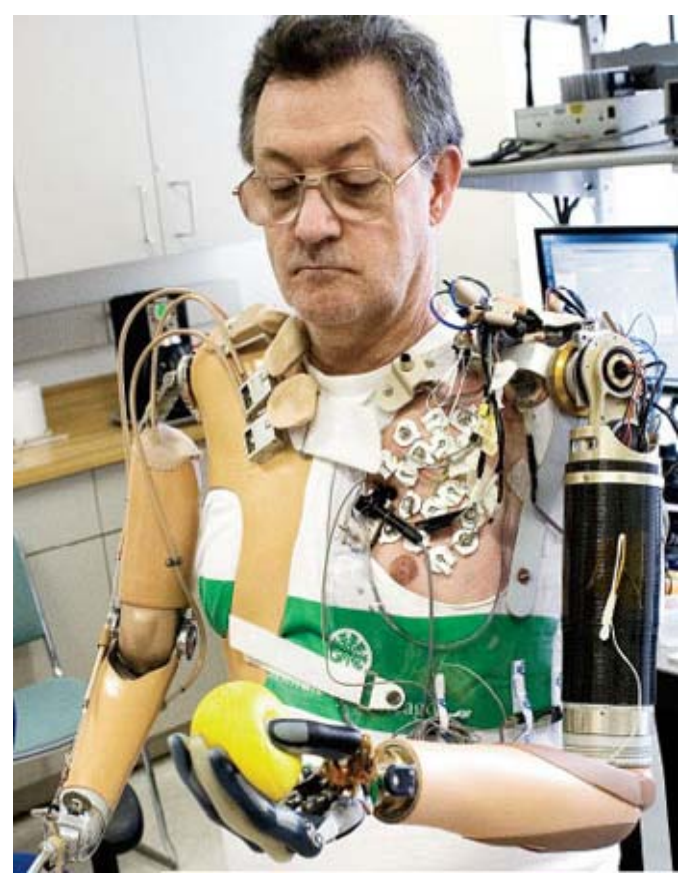

Figure 1-1. DARPA Arm Project ${ }^{1}$

The results of the DARPA arm project so far have been encouraging and have received wide coverage in the national press, including special attention from former President George W. Bush, as shown in Figure 1-2. The recent conflicts in Afghanistan and Iraq have also raised awareness about lower extremity amputation due to the large number of soldiers who have been injured by improvised explosive devices (IEDs) and roadside bombs.

\footnotetext{
${ }^{1}$ Image from “DARPA's Better Bionic Arm: Our Most Limb-Like Prosthetic," Popular Mechanics, July 2007, http://www.popularmechanics.com/science/health_medicine/4218218.html
} 


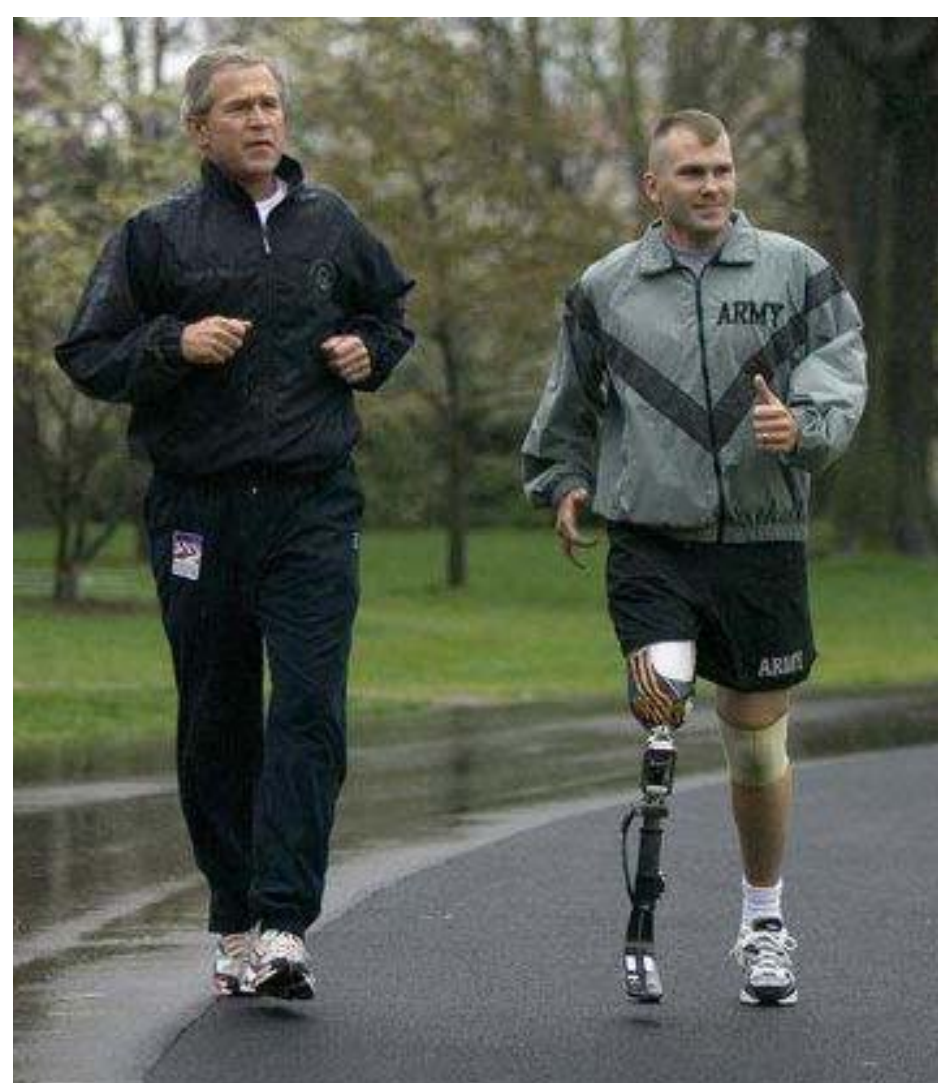

Figure 1-2. President Bush Jogging with Amputee Soldier ${ }^{2}$

There are several upper extremity myoelectrically-controlled prostheses currently available. The Motion Control "Utah Arm 3", as shown in Figure 1-3, is clinically available for above-elbow amputees. The original Utah Arm was released in 1981, with Utah Arm 2 released in 1987 and the most recent design released in 2004. It enables simultaneous hand and arm control and allows an active lift of $1 \mathrm{~kg}$. Otto Bock also has a myoelectrically controlled arm called the DynamicArm and several myoelectric hands and grippers ${ }^{3}$.

\footnotetext{
${ }^{2}$ Image from "White House Photo Essay 1," April 14 2004, http://www.whitehouse.gov/news/releases/2004/04/images/20040414-7 a5bu9736-677v.html

${ }^{3}$ Otto Bock myoelectric products, http://www.ottobock.com/cps/rde/xchg/ob_com_en/hs.xsl/384.html
} 


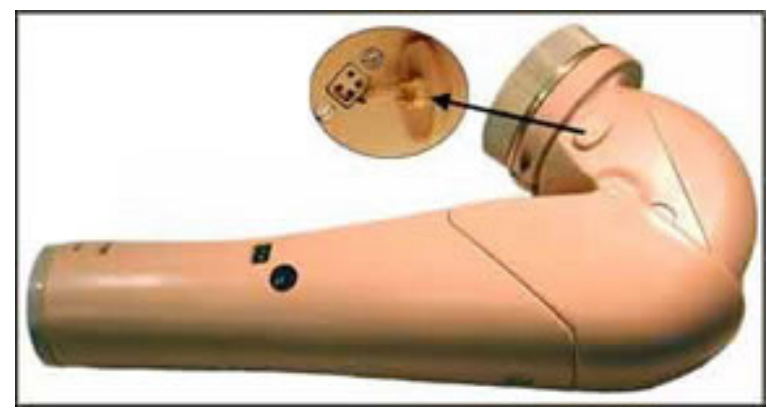

Figure 1-3. Motion Control Utah Arm 3 (UA3) ${ }^{4}$

This project aims to enable intelligent natural control of next-generation powered lower- and upper-extremity prostheses by developing a battery-less, long-term implantable EMG sensor with wireless powering and data telemetry capabilities suitable for measuring myoelectric control signals from residual limb muscles. The implantable device will be designed for shallow subfascial implantation on to the surface of target muscles.

The wireless implantable epimysial EMG-sensing system is designed to measure myoelectric signals from the surface of muscles in an amputee's residual limb and transmit the digitized signal to an external transceiver for analysis and adaptive prosthetic control. An external wireless powering and data telemetry module housed in a prosthetic socket interfaces with the implanted electronics. Placement of the subcutaneous wireless sensing microsystem directly on the target muscle surface is expected to significantly reduce movement artifacts and obviate other detrimental factors associated with skin surface EMG recording as described above. This approach also has the advantage of allowing a prosthesis user to wear a socket liner for enhanced comfort, where previous generation systems utilizing electrodes in the socket preclude the use of a socket liner.

\footnotetext{
${ }^{4}$ Utah Arm 3 (UA3), http://www.utaharm.com/ua3.php
} 
The wireless EMG-sensing microsystem is expected to substantially enhance the biomimetic performance of myoelectrically-controlled prosthetic limbs for amputees. There are currently no commercially available myoelectrically-controlled lower-limb prostheses. Motivating considerations are one reason for this, as most amputations involve the upper extremities. Many lower extremity amputees achieve a high level of function with the currently available state-of-the-art lower-extremity microprocessorbased prostheses such as the Otto Bock C-Leg, and mechanical prostheses such as the Össur ${ }^{5}$-brand products including the Flex Foot, which has gained attention for its use in the Paralympics. The C-Leg is limited in that it operates in discrete, user-selectable modes. Control of the prosthesis mode is achieved through a hand-held or body-powered switch. Neural control from EMG signals will allow seamless mode switching and task transition, as well as increased opportunities to control gait such as control of ankle position. The increased bandwidth, enhanced sensitivity, and reduced susceptibility to recording artifacts of a fully implantable system will provide an accurate control signal enabling the next generation of EMG-controlled upper- and lower-extremity prostheses.

\subsection{Thesis Organization}

This thesis is organized as follows. Chapter 1 introduces the research project by presenting the motivation and goals of the project. Chapter 2 provides an overview of the applications for myoelectric control and the design requirements of the project. Chapter 3 presents an overview of the EMG sensing microsystem architecture. Chapter 4 illustrates

the design of the integrated CMOS EMG sensing electronics. Chapter 5 presents the

${ }^{5}$ Össur Prosthetics http://www.ossur.com/?PageID=12613 
design of the remote RF powering and data telemetry system. Chapter 6 shows system measurement and characterization results, and in vivo laboratory animal test data. Chapter 7 summarizes the research work with conclusions and future work. 


\section{Chapter 2}

\section{Wireless Implantable EMG Sensor for Myoelectrically-}

\section{Controlled Prosthetics}

\subsection{Overview of Wireless EMG-Sensing Application}

EMG is a critical biopotential signal for prosthesis control applications. By sensing muscle contractions and recording EMG activity, a specific neural control signal of interest can be measured. EMG recording can provide a viable control signal for an amputee's prostheses when the muscle of interest is still innervated and intact. For example, facial EMG signals have been used as a control source for individuals with high spinal cord injuries. EMG signals are also commonly used as a control signal for myoelectric hand prostheses. In the case of the DARPA Arm project, the muscles of interest are no longer intact, but the controlling nerves are reattached to healthy pectoral muscles.

An implantable EMG sensor for upper-extremity prosthesis control is under development for use in the DARPA Arm, as well as for other applications. This system is designed to fit into a cylindrical ceramic package similar to the Alfred Mann Foundation's BION device [2]. The first generation device, BION1, shown in Figure 2-1, is designed to continuously perform functional electrical stimulation and is implanted via a large gage hypodermic needle. The BION2 device will add EMG sensing capability to 
enable transmission of muscle activity data for use as a prosthetic control signal for feedback in FES systems. The BION2 system is designed to energize several implant devices with one large external coil that wraps around the residual limb. Each individual implant is assigned a time slot for data transmission and share access to a single $40 \mathrm{~kb} / \mathrm{s}$ outward data channel[3], so EMG measurement signal bandwidth and resolution are limited. To maintain a high resolution EMG waveform, the serial data rate of BION2 would have to be increased, or an alternate outward data link topology should be.

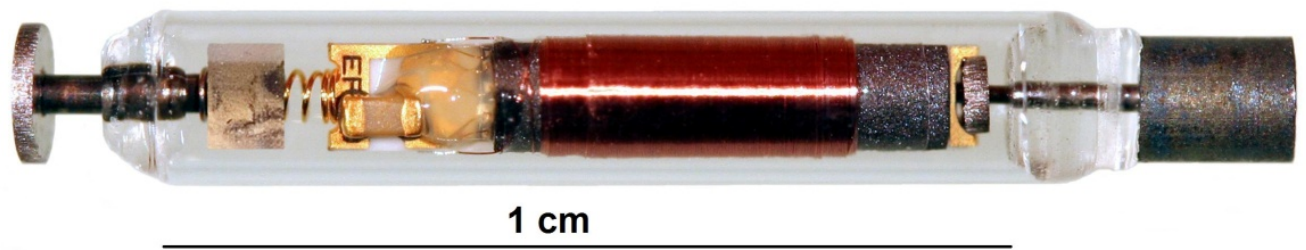

Figure 2-1. BION1 Package for Functional Electrical Stimulation ${ }^{6}$

An implantable wireless EMG sensing microsystem has been developed that can be implanted under the subcutaneous fat and secured to the surface of muscles in the residual limb of an amputee. The system is designed to target broad, flat, and surgically accessible muscles of interest in the residual limbs of both lower- and upper-extremity amputees. Subfascial implantation is performed with a simple, minimally-invasive surgical technique. Shallow implantation allows for a relatively high RF power and data coupling, enabling continuous real-time, high-resolution EMG signal monitoring.

An overview of the wireless EMG control application is shown in Figure 2-2. The implantable device is transcutaneously and wirelessly powered by a radio-frequency (RF) signal from an external transceiver housed in a prosthetic socket. The integrated

\footnotetext{
${ }^{6}$ Image from "BIONic Reanimation of Paralyzed Limbs," http://qb3.org/pdfs/Loeb.pdf
} 
electronics rectify and regulate the received RF energy into a stable DC supply. The sensing electronics amplify, digitize, and transmit EMG signals to the transceiver over the same wireless link as the RF powering system using a passive digital data telemetry scheme. The received digital EMG data may then be processed by a microprocessor and used to achieve adaptive control of the prosthetic limb.

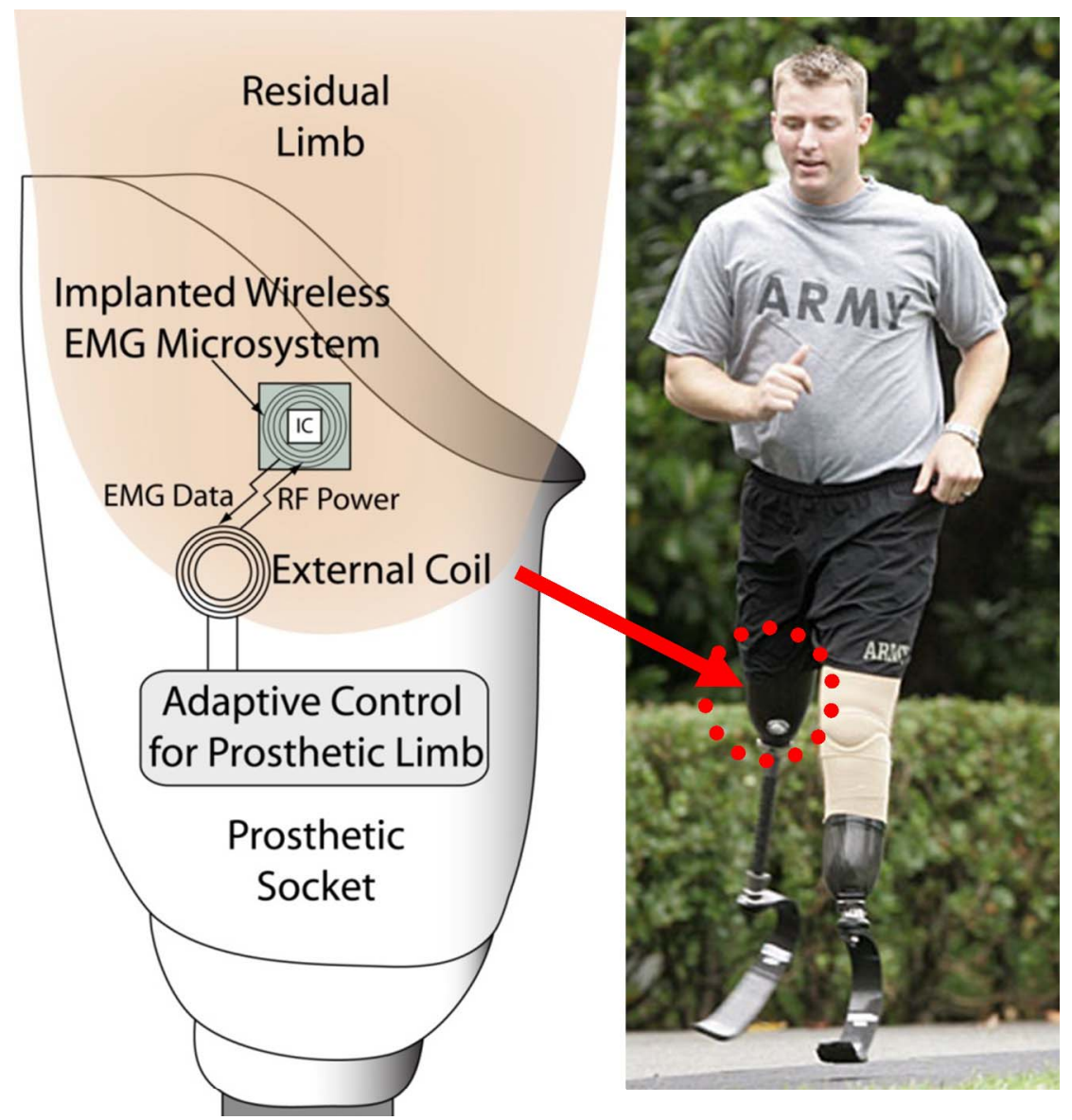

Figure 2-2. Wireless EMG Prosthesis Control Application ${ }^{7}$

\subsection{Design Requirements}

\footnotetext{
${ }^{7}$ Image from “Amputee Iraq vet fulfills wish, jogs with Bush,” USA Today, June 27 2006, http://www.usatoday.com/news/washington/2006-06-27-bush-jog_x.htm
} 
EMG signals of interest for prosthetic control have a bandwidth of approximately $1 \mathrm{kHz}$ with maximum energy around $250 \mathrm{~Hz}$ and maximum differential signal amplitude of $10 \mathrm{mV}_{\mathrm{PP}}$. The minimum detectable differential EMG signal is approximately 5 to 10 $\mu \mathrm{V}_{\mathrm{PP}}$. Implantable epimysial Pt-Ir EMG sensing electrodes developed by the Cleveland FES Center and now manufactured by Ardiem Medical, Inc. are chosen for this application as they are preferred for the broad, flat, surgically accessible muscles in the residual limb. This system is also compatible with bipolar intramuscular EMG electrodes and potentially other EMG-sensing electrodes. Epimysial electrodes are implanted on the surface of a target muscle with a small incision, allowing for a fast recovery. In addition, the placement of epimysial electrodes directly under the skin enables short-distance RF powering and data telemetry capability.

The implantable microsystem is designed to be powered wirelessly through a near-field RF inductive link. A Class-E switching power amplifier is chosen for its high DC to RF power conversion efficiency. The Class-E amplifier drives a series-resonant tank, including a 2-cm diameter, 10-turn air-core spiral coil made of 24-gage enameled copper speaker wire and coated in epoxy. The implantable ASIC is interfaced with a parallel-resonant tank, including a 6-mm diameter, 30-turn spiral coil, which is made of 36-gage enameled copper speaker wire and designed to fit around the ASIC chip. When the external primary and implantable secondary coils are brought in to an approximate alignment, near-field inductive coupling enables transfer of energy from the primary coil to the secondary coil. The resonant system is optimized for wireless power transfer efficiency and both tanks are tuned to resonate at $8 \mathrm{MHz}$. The inductive link is designed to operate at up to $2 \mathrm{~cm}$ coil separation, corresponding to a typical thickness for the 
intervening tissue between an implanted sensor and the socket surface. The RF power received by the implantable device is converted into stable $2.1 \mathrm{~V}$ and $2.7 \mathrm{~V}$ supply lines by an integrated RF-DC rectifier and regulator.

The objective is to capture as much information about the EMG waveform as possible so that future amputee intent or gait state identification algorithms can take advantage of the entire EMG signal to derive an optimized and efficient prosthetic control signal. This EMG signal can be made available as a digital bit-stream suitable for interfacing with a PC or microcontroller.

The implantable epimysial EMG-sensing electrodes are interfaced with a lownoise and low-power CMOS differential amplifier based on a subthreshold or moderate inversion design [4]. The amplifier is AC-coupled to the sensing electrodes with a $-3 \mathrm{~dB}$ bandwidth of $1 \mathrm{kHz}$ to capture a high-fidelity EMG signal for adaptive control of a prosthetic limb. The low-noise amplifier is designed to consume approximately $5 \mu \mathrm{A}$ from a $2 \mathrm{~V}$ supply. The differential design achieves a high common-mode rejection ratio critical for measuring in vivo real-time physiological signals. The output of the low-noise amplifier is sampled by a switched-capacitor gain stage followed by an 11-bit cyclic differential ADC [5].

The digital output of the ADC is Manchester-encoded and transmitted to an external transceiver housed in the prosthetic socket by passive digital phase-shift keying (PSK) modulation scheme through the same wireless link used for RF powering. Digital PSK modulation is achieved by detuning the secondary (implanted) tank with a switching capacitor. This detuning action reflects a complex impedance to the primary tank of the external transceiver, causing a phase shift in the tank current relative to the $8 \mathrm{MHz}$ clock 
driving the Class-E amplifier. This phase modulation is detected by a phase detection circuit in the external transceiver, which mixes the Class-E clock signal with the primary tank voltage to recover the digital information encoded in the reflected phase shift.

The received Manchester-encoded data recovered by the external transceiver module is then sampled by a data acquisition card interfaced with a desktop PC. Custom software written in the LabVIEW language performs Manchester decoding, locks on to the digital data stream, displays the digitized EMG waveform, performs parity checking, and $\operatorname{logs}$ the received data to disk for further analysis. Future systems may perform additional processing on these data in real-time to form a control signal for next generation prosthetic devices.

\subsection{Summary}

In this chapter, a summary of EMG sensing applications has been presented. In addition, the design requirements for a fully implantable wireless EMG sensing microsystem have been illustrated. This includes a description of the EMG sensing electrodes, the wireless inductive powering technique, the EMG-sensing and digitizing electronics, the passive telemetry link, and an overview of the requirements for interfacing with the digital EMG data. The following chapters will present a complete and detailed system design. 
Chapter 3

\section{Wireless Implantable EMG Sensing Microsystem Overview}

\subsection{Overview}

The wireless implantable EMG sensing microsystem, as shown in Figure 3-1, consists primarily of an implantable digitizing EMG sensor, which is interfaced through an RF near-field inductive link to an external transceiver providing RF data receiving capability as well as RF powering [5-7]. The implantable sensor employs differential EMG sensing electrodes such as Pt-Ir epimysial electrodes, an RF coil, and a customdesigned ASIC. The ASIC includes a low-noise and low-power EMG amplifier, an 11bit differential cyclic analog-to-digital converter (ADC), wireless RF-DC power conversion electronics with digital phase-shift keying data telemetry and control logic circuitry. The external transceiver is composed of a Class-E switching power amplifier, transmitting RF coil and discrete PSK demodulating electronics. This chapter presents an overview of each of these system blocks. 


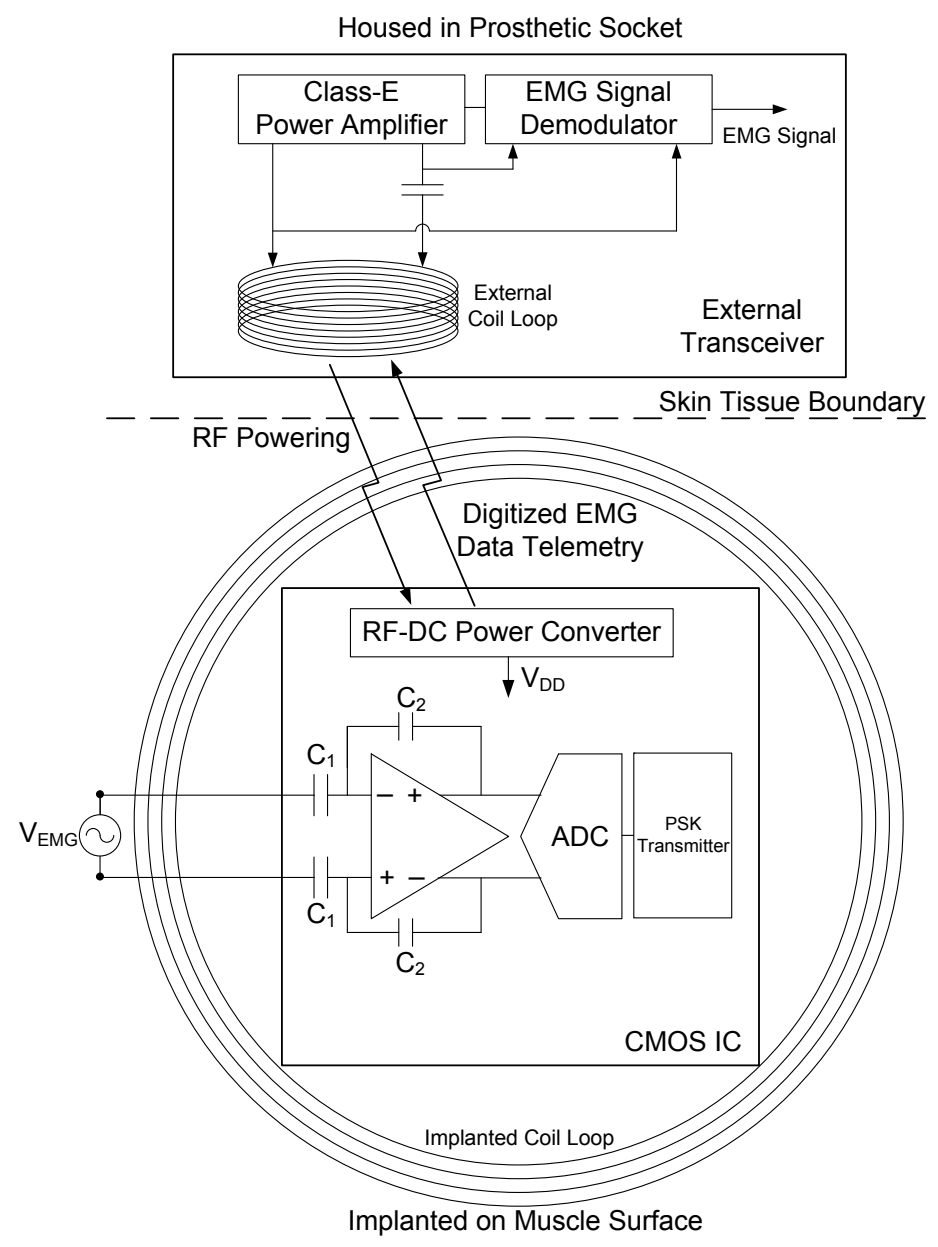

Figure 3-1. EMG Sensing System Overview

\subsection{Low-Power and Low-Noise Front-End Amplifier}

An integrated CMOS low-noise and low-power differential amplifier based on a subthreshold design has been implemented to interface with EMG sensing electrodes. EMG signals of interest for prosthetic control exhibit a bandwidth of approximately 1 $\mathrm{kHz}$ with maximum energy around $250 \mathrm{~Hz}$, and a maximum differential signal amplitude of $10 \mathrm{mV}_{\mathrm{PP}}$. The minimum detectable EMG signal amplitude is typically from $5 \mu \mathrm{V}_{\mathrm{PP}}$ to $10 \mu \mathrm{V}_{\mathrm{PP}}$. The front-end amplifier is capacitively coupled to the sensing electrodes with a designed $-3 \mathrm{~dB}$ bandwidth of $1 \mathrm{kHz}$. DC baseline stabilization with a high-pass corner 
frequency of approximately $20 \mathrm{mHz}$ is achieved by placing a MOSFET-based active resistive element in the amplifier feedback path in parallel with the feedback capacitor [4]. A fully differential architecture achieves a high common-mode rejection ratio, critical for sensing in vivo real-time physiological signals.

The EMG interface amplifier is designed as a telescopic operational transconductance amplifier (OTA) with a PMOS differential input pair operating in the subthreshold or moderate inversion region with a $2 \mathrm{~V}$ supply for low power dissipation. A continuous-time output common-mode feedback circuit is implemented to set the output common-mode level to $0.7 \mathrm{~V}$. The amplifier is designed to achieve a closed-loop gain of 80 set by on-chip capacitors ratio of $\mathrm{C}_{1} / \mathrm{C}_{2}$.

A switched-capacitor $2.5 \mathrm{x}$ differential gain stage and 11-bit differential cyclic ADC that were previously designed for another project in the same AMI $1.5 \mu \mathrm{m}$ CMOS process [5] have been adapted for use in the prototype microsystem design. The $2.5 \mathrm{x}$ gain stage was designed specifically to drive the ADC, and the front-end amplifier is designed with an appropriate gain and output swing to properly interface with the full input range of the $2.5 \mathrm{x}$ gain stage. The front-end amplifier and 2.5x gain stage are shown in Figure 3-2. 


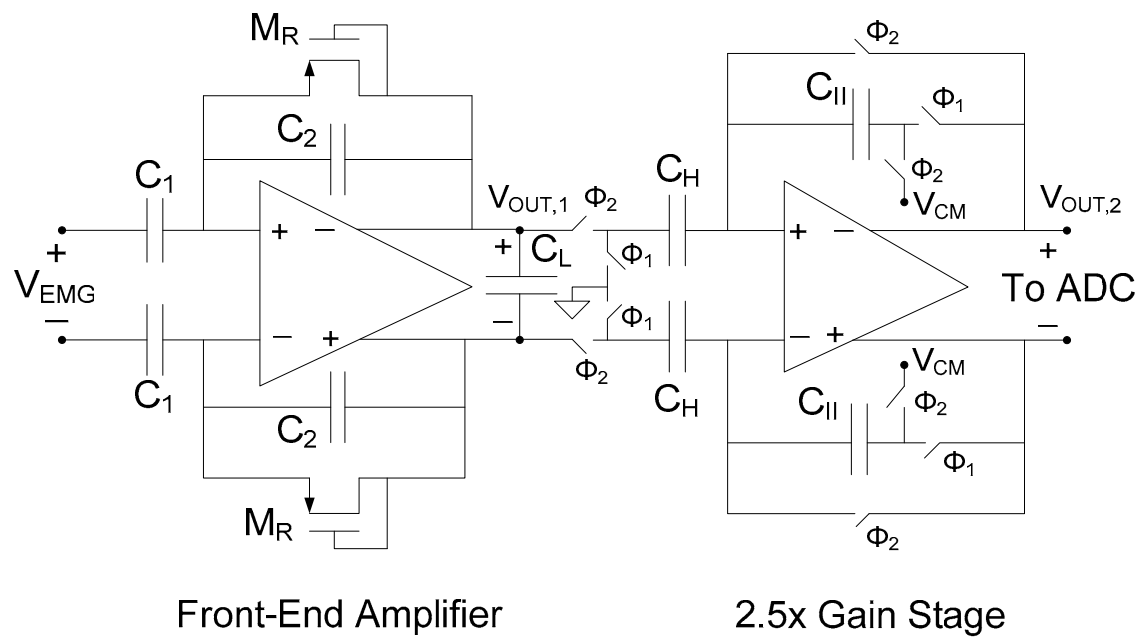

Figure 3-2. Interface Electronics

\subsection{1-bit Differential Cyclic ADC}

The output of the $2.5 \mathrm{x}$ gain stage is digitized by an 11-bit cyclic ADC. This topology has been selected for its low power dissipation, adequate resolution, small silicon area, and simplicity of design [5].

Figure 3-3 shows a cyclic ADC architecture including a sample-hold circuit, a multiply-by-two amplifier, a subtraction circuit, and a comparator. In a practical implementation, the subtraction circuit and the multiply-by-two amplifier can be combined together, which is commonly referred as a multiplying DAC (MDAC). First, $V_{\text {in }}$ is sampled as shown in Equation 3-1, and is then compared with a zero threshold to decide the most significant bit (MSB).

$$
V(1)=V_{\text {in }}
$$

Next, $V_{\text {in }}$ is multiplied by two and added to or subtracted from a reference voltage, $V_{\text {Ref }}$, depending on the value of MSB, thus resulting in a residue voltage of $2 V_{i n} \pm V_{R e f}$ as 
shown in Equation 3-2. The residue voltage is then processed by the same loop until the least significant bit (LSB) is determined.

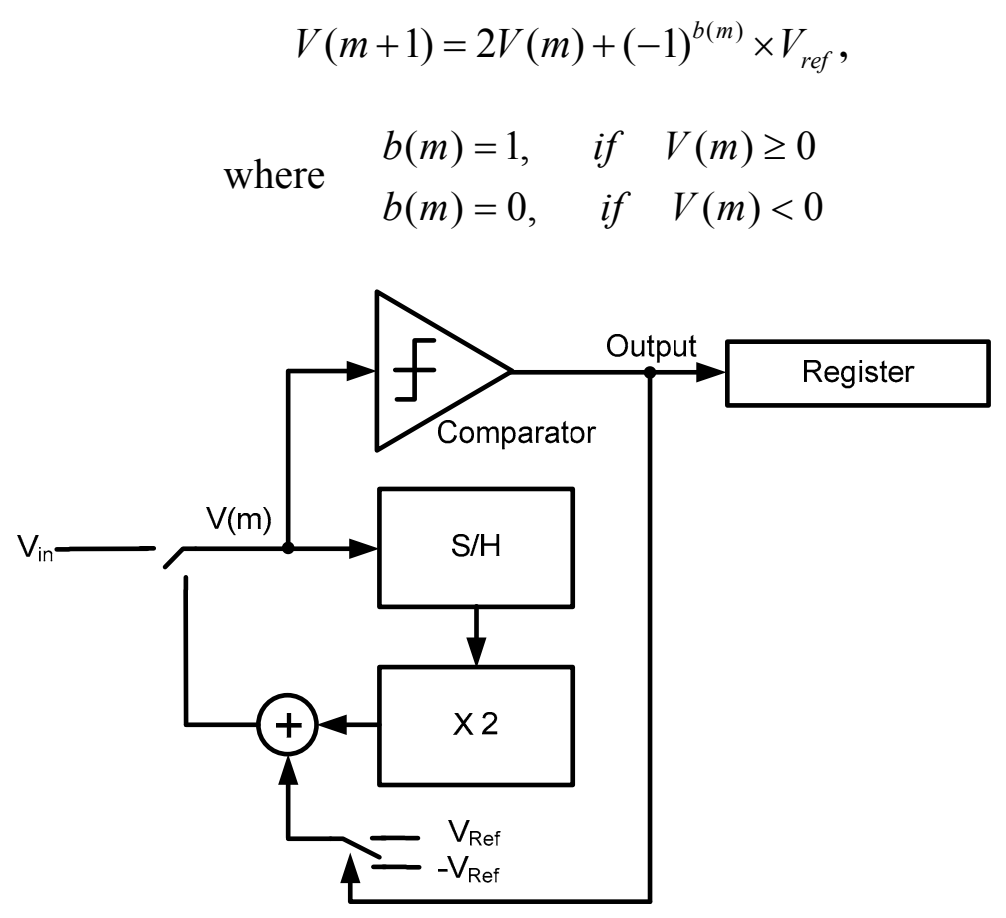

Figure 3-3: Cyclic ADC architecture.

This design employs a ratio-independent multiply-by-two scheme in the multiplying DAC (MDAC) to avoid capacitor matching limitations. This is achieved by doublesampling the input voltage followed by exchanging the sampling and integrating capacitors. The multiply-by-two amplifier requires four phases and resets in each clock cycle to suppress the amplifier DC offset and $1 / f$ noise. The sample-and-hold circuit requires two phases to operate and also resets on phase 1 to reduce the effect of DC offset and $1 / f$ noise.

The ADC output is represented in the redundant signed digit (RSD) scheme. This improves the ADC's immunity to errors caused by offset voltages and comparator inaccuracy. The $\mathrm{ADC}$ decision levels of $\mathrm{V}_{\mathrm{L}}$ and $\mathrm{V}_{\mathrm{H}}$ may vary over a wide range without 
introducing missing or redundant codes, which would cause linearity degradations. Figure 3-4 shows a schematic of the cyclic ADC employing redundant signed digit (RSD) and ratio-independent multiply-by-two schemes. Figure 3-5 shows the corresponding cyclic ADC timing diagram.

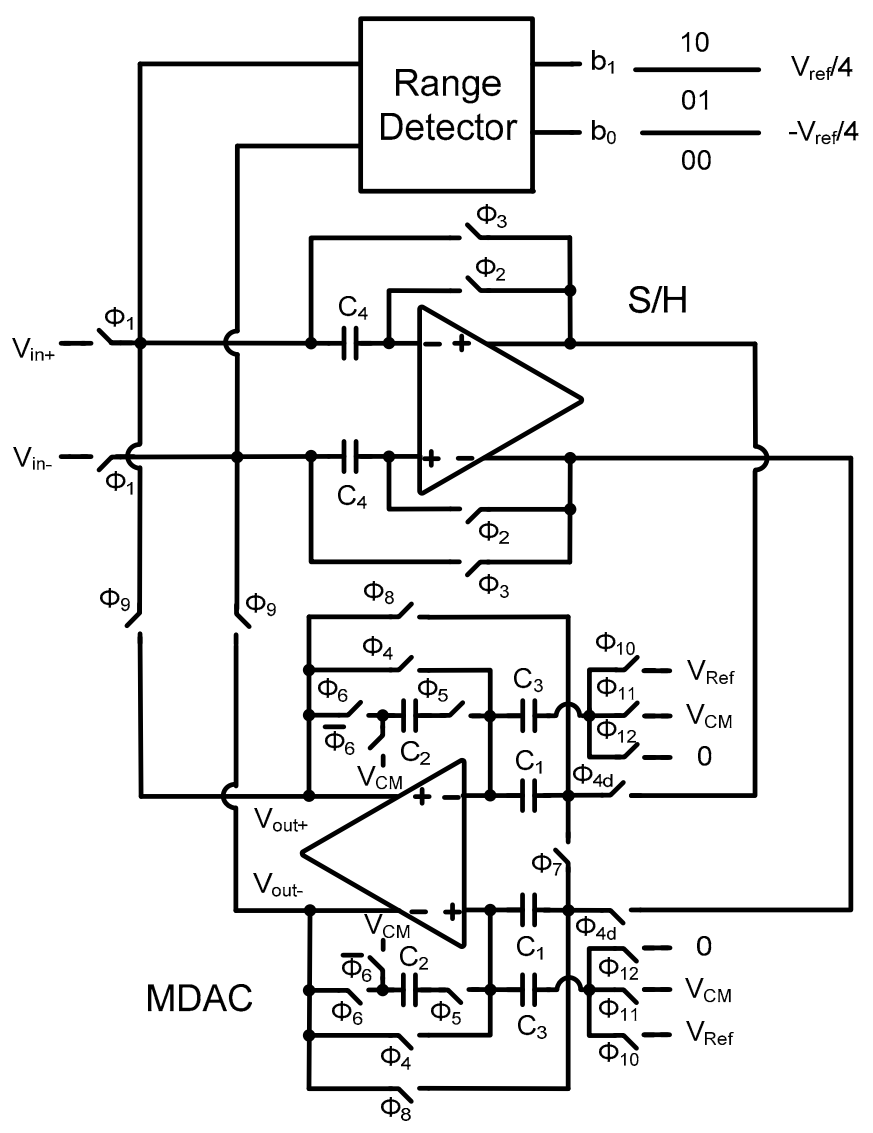

Figure 3-4. Schematic of Cyclic ADC 


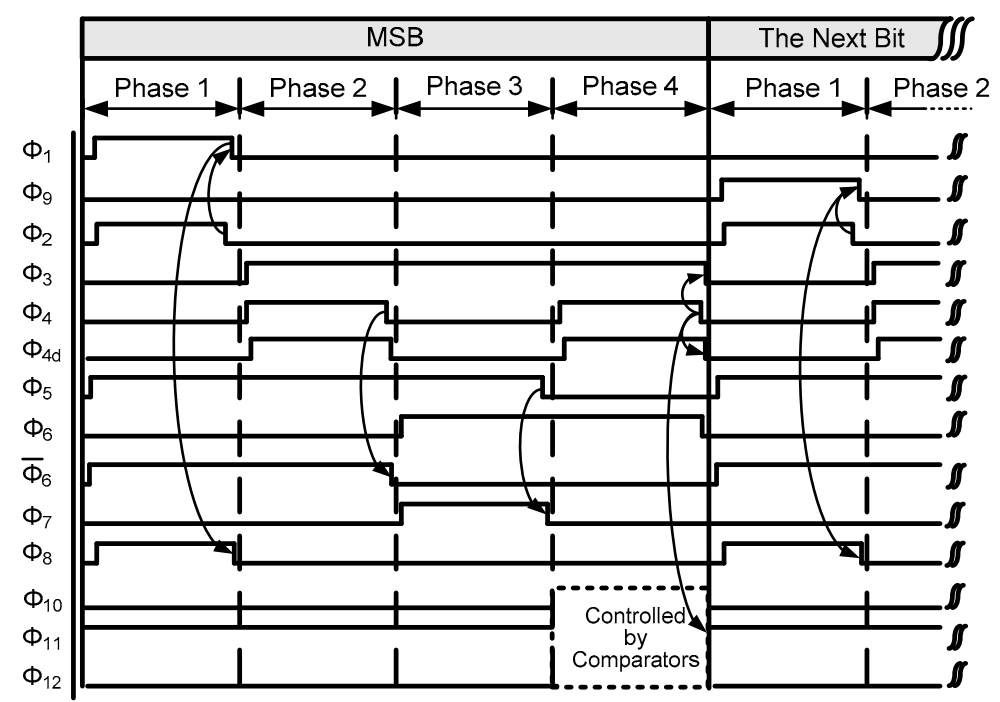

Figure 3-5. Cyclic ADC Timing Diagram

\subsection{Remote RF Powering System}

In order to wirelessly transfer power to the implantable EMG sensor, inductively coupled RF coils are employed to create a near-field link over a distance of 1-2 cm operating at $8 \mathrm{MHz}$. The $8 \mathrm{MHz}$ operating frequency is selected for a number of reasons. First, it is advantageous to select an RF operating frequency below $20 \mathrm{MHz}$ in order to ensure a minimal RF power dissipation in the tissue between the coils, although higher frequencies of operation may be used with degraded wireless power transfer efficiency and potentially higher tissue warming effects. [6] Next, coupling performance generally increases with higher frequency until the self-resonant frequencies of the inductive coils are approached. The small RF coils used in this system exhibit relatively high selfresonant frequencies in the mid-tens of $\mathrm{MHz}$ range. An inductive coupling performance investigation, as described in Chapter 5, reveals that $8 \mathrm{MHz}$ is an optimal operating point for wireless power transfer for inductive coils similar to those used in the final prototype 
design. It is critical to maximize wireless power transfer efficiency in order to consume a minimal amount of power at the external power amplifier in order to maximize battery operation time in a prosthetic application.

A Class-E switching power amplifier topology [7] is chosen for its high DC to RF power conversion efficiency. The implantable (secondary) inductor coil is designed to have an outer diameter of $6 \mathrm{~mm}$ so that it can be placed around the EMG sensing ASIC and ultimately packaged with the Pt-Ir foil EMG electrodes. A spiral coil with $2 \mathrm{~cm}$ diameter is designed for the external (primary) coil for ease of mounting in a prosthetic socket, optimal planar alignment, and maximized wireless power transfer efficiency to the implanted electronics. In this case, an optimal planar alignment means that the coil geometry is designed to be small compared to the typical circumference of the prosthetic socket. This way, the coil will not be forced to bend or conform to the contour of the socket, but instead can remain planar and parallel to the implanted coil.

Figure 3-6 shows a plot of relative power transfer efficiency, compared to perfect alignment (defined as $100 \%$ ), as a function of lateral displacement offset with a fixed coils separation distance of $1.3 \mathrm{~cm}$. If the displacement offset is constrained to an area roughly equivalent to the size of the $2-\mathrm{cm}$ secondary coil, the efficiency reduction is less than $20 \%$ from maximum. If the secondary coil is misaligned by a distance greater than the radius of the primary coil, the efficiency drops off more quickly. For example, at a 2$\mathrm{cm}$ offset from perfectly aligned, the efficiency drop can be as high as $80 \%$ from maximum. Exact parallel coils alignment is not required, as the system will function well with alignment angle errors of less than about 45 degrees. If the alignment angle error increases beyond 45 degrees, the power transfer efficiency drops rapidly. 


\section{Displacement Offset Sensitivity @ 1.3 cm}

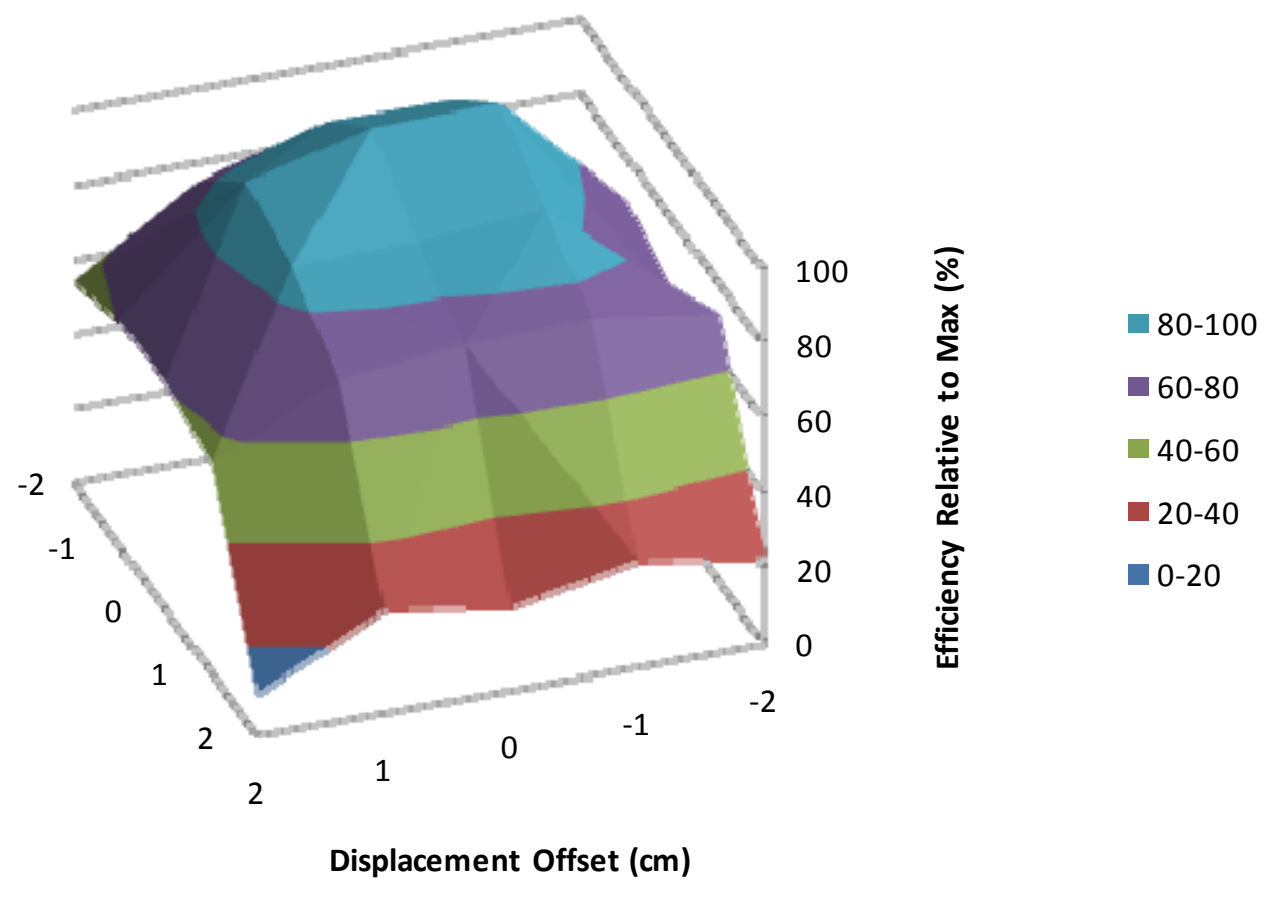

Figure 3-6. Wireless Powering Displacement Sensitivity

The design of the coil geometry and optimization of the number of turns in the coil to maximize wireless transfer efficiency will be considered in Chapter 5.

\subsection{Phase-Shift Keying Passive Data Telemetry System}

Passive digital phase-shift keying (PSK) modulation is used to wirelessly transmit the EMG sensor data to the external transceiver over the same inductive link used by the RF powering system. PSK modulation is achieved by digitally detuning the secondary (implanted) LC tank with a switching capacitor, $\mathrm{C}_{\mathrm{SW}}$, as shown in Figure 3-7. By switching the capacitor in parallel with a nominal tuning capacitor $\mathrm{C}_{2}$, the secondary tank is detuned from the operating frequency and a complex impedance is reflected to the 
primary tank. This causes the current flowing in the primary tank to have a phase shift relative to the $8 \mathrm{MHz}$ clock reference signal. The Manchester-encoded digitized EMG data bit-stream is used to drive this switching operation. The PSK data can be recovered by mixing the tank voltage with the $8 \mathrm{MHz}$ clock signal, which demodulates the PSK data to baseband. The mixer output is low-pass filtered and then passed through a comparator to produce the recovered Manchester-encoded digital data. The bit-stream is oversampled and decoded with a state machine implemented in custom LabVIEW interface software. Oversampling is required to recover the data clock embedded in the signal through the Manchester encoding process. A general design diagram of the RF powering and PSK data telemetry system is presented in Figure 3-7. The detailed PSK system design is presented in section 5.5.

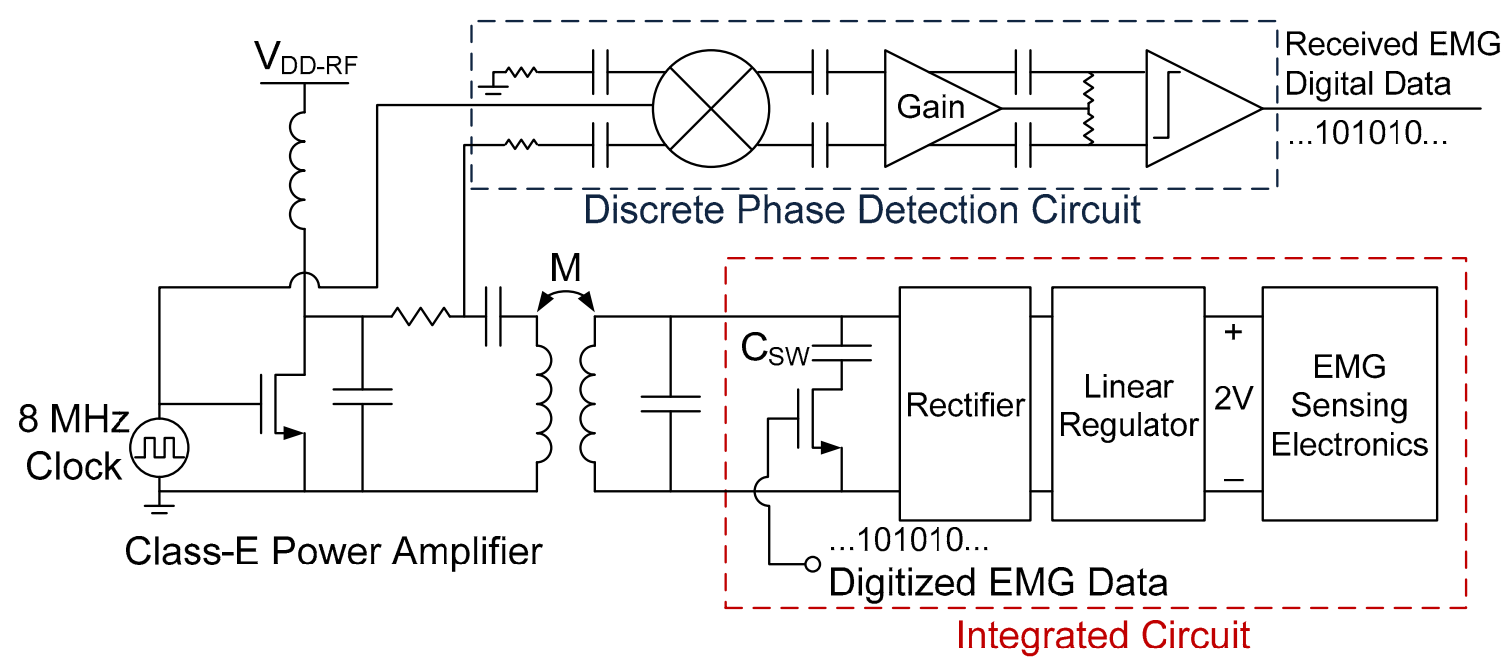

Figure 3-7. RF Powering and PSK Data Telemetry System

\subsection{Summary}

An overview of the EMG-sensing microsystem is presented in this chapter. Epimysial electrodes interface to muscles of interest providing a differential EMG signal. 
Custom-designed integrated EMG interface electronics consist of a low-noise and lowpower differential amplifier, a $2.5 \mathrm{x}$ gain stage, and a differential cyclic ADC. The sensing electronics are powered wirelessly through an RF inductive link. On-chip rectification and regulation circuits provide a stable DC power supply to power the integrated EMG sensing electronics. Passive digital PSK telemetry enables wireless data transmission over the same inductive link used for wireless powering. Digital state machine control is implemented with an on-chip digital control block. A detailed design of the low-noise and low-power EMG interface amplifier and the RF powering and data telemetry link will be presented in the following chapters. 


\section{Chapter 4}

\section{Integrated CMOS EMG Sensing Electronics}

\subsection{Front-end Amplifier Overview}

The EMG sensing electronics are designed to obtain a sufficiently high-resolution EMG waveform suitable for prosthesis control. The front-end amplifier is designed to properly interface EMG signals sensed by differential EMG-recording electrodes to the following $2.5 \mathrm{x}$ gain stage. The front-end amplifier is designed as a fully differential, capacitively coupled operational transconductance amplifier (OTA), as shown in Figure 4-1.

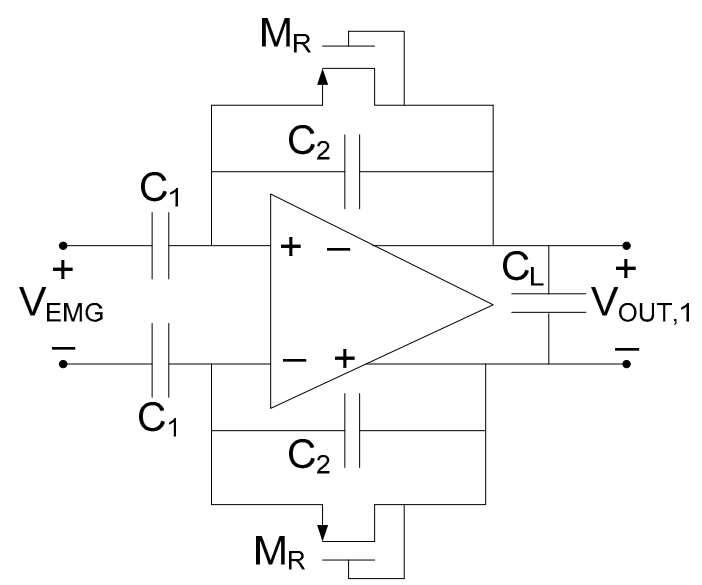

Figure 4-1. Front-end Amplifier

Typical EMG signals of interest have a differential signal range from a minimum detectable level of 5 to $10 \mu \mathrm{V}_{\mathrm{PP}}$ to a maximum differential signal of $10 \mathrm{mV}_{\mathrm{PP}}$ within a 1 $\mathrm{kHz}$ bandwidth. The full scale differential input range of the differential cyclic ADC of 
$2 \mathrm{~V}_{\mathrm{PP}}$, along with the $2.5 \mathrm{x}$ gain stage, sets the required closed-loop gain of the front-end amplifier to be 80 , thus achieving a total signal gain of $80 \times 2.5=200$. This amplifies a maximum differential input signal of $10 \mathrm{mV}_{\mathrm{PP}}$ to the differential full-scale $2 \mathrm{~V}_{\mathrm{PP}}$ input range of the $\mathrm{ADC}$.

The DC biasing and baseline stabilization are achieved by using MOSFET-based resistive elements in the feedback path. The closed-loop gain of 80 is set by on-chip capacitors ratio, as shown in Equation 4-1.

$$
A_{C L}=\frac{1}{f}=\frac{C_{1}+C_{2}}{C_{2}} \approx \frac{C_{1}}{C_{2}}=80
$$

The Poly1 to Poly2 capacitance density in the AMI $1.5 \mu \mathrm{m}$ CMOS process of $0.59 \mathrm{pF}$ / $\mu \mathrm{m}^{2}$ drives the selection of the nominal $\mathrm{C}_{1}$ and $\mathrm{C}_{2}$ capacitors size. The minimum drawn capacitor size is $6 \lambda \times 6 \lambda$, which has a capacitance value of $13.6 \mathrm{fF}$. It is desirable to design $C_{2}$ to be larger than the minimum size. The $C_{2}$ capacitance should be larger than the capacitance contribution from the MOSFET-based feedback resistors, which add in parallel with $C_{2}$ and therefore will affect the closed-loop voltage gain. A large value of $C_{2}$ will also minimize the effects of fringing capacitance and thus improve matching. $C_{2}$ is designed to be $2 \times 12 \lambda \times 12 \lambda$, which has a capacitance value of $100 \mathrm{fF}$. This in turn sets $C_{1}=8 \mathrm{pF}$.

\subsection{Differential Operational Transconductance Amplifier Design}

The EMG front-end amplifier is designed as a fully-differential telescopic operational transconductance amplifier (OTA). The OTA core schematic is shown in Figure 4-2. 


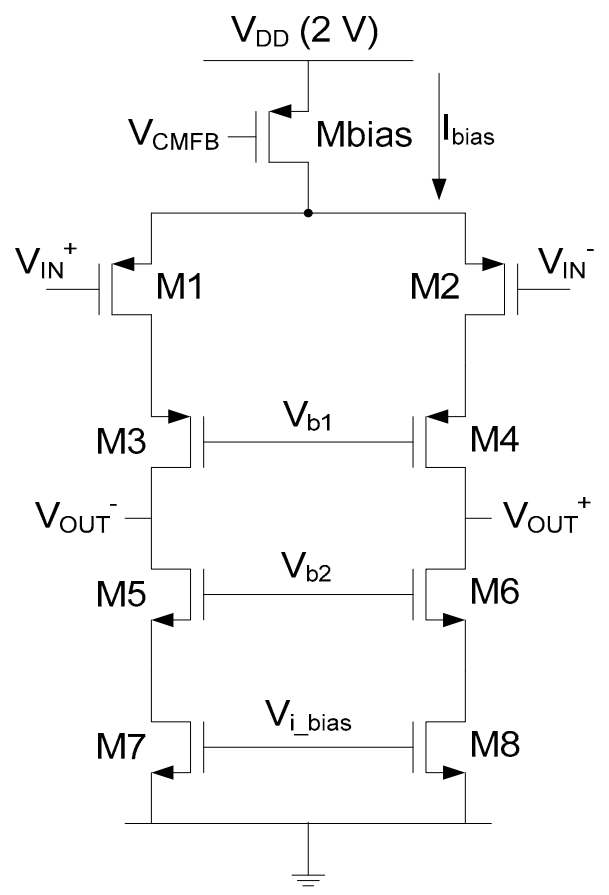

Figure 4-2. OTA Circuit Schematic

The differential input pair is designed with PMOS transistors for a reduced $1 / f$ noise contribution. The input pair is biased in the moderate inversion region to minimize power dissipation. The front-end amplifier is designed such that the output thermal noise contribution is less than $1 / 2 \mathrm{LSB}$ of the ADC when referenced to ADC input, which is 0.5 $m V_{\text {RMS. }}$. The total output thermal noise voltage requirement, $v_{o N}$, can be divided by the $2.5 \mathrm{x}$ gain stage and the OTA noise gain to obtain the OTA input-referred thermal voltage noise power spectral density, $v_{i N}$, as shown in Equation 4-2,

$$
v_{i N}=\frac{v_{o N} / 2.5}{\left(C_{T} / C_{2}\right)}=\frac{0.5 \mathrm{mV}_{R M S} / 2.5}{81}=2.5 \mu \mathrm{V}_{R M S},
$$

where $C_{T}$ is the total capacitance at the amplifier input.

The amplifier input-referred thermal noise is dominated by the input differential pair and the NMOS current source. The NMOS current source must be considered since its differential noise contribution can be considerable relative to the input differential 
pair, depending on the ratio of the NMOS current source small-signal transconductance, $g_{m 7,8}$, to the input differential pair small-signal transconductance, $g_{m 1,2}$. A reasonable assumption of $g_{m 1,2}$ as approximately twice $g_{m 7,8}$ is made to express the input-referred thermal voltage noise power spectral density as a function of $g_{m 1,2}$, as shown in Equation $4-3$,

$$
\frac{v_{i N}}{\sqrt{\Delta \mathrm{f}}}=\sqrt{2 \cdot \frac{2}{3} \frac{4 k T}{g_{m 1,2}}\left(1+\frac{g_{m 7,8}}{g_{m 1,2}}\right)} \approx \sqrt{\frac{16}{3} \frac{k T}{g_{m 1,2}}\left(1+\frac{1}{2}\right)} .
$$

The integrated input-referred thermal noise from Equation 4-2 sets the inputreferred thermal voltage noise power spectral density, and therefore the required $g_{m}$ of the input pair transistors, as shown in Equation 4-4.

$$
\frac{v_{i N}}{\sqrt{\Delta \mathrm{f}}}=\frac{2.5 \mu \mathrm{V}_{\mathrm{RMS}}}{\sqrt{1 \mathrm{kHz} \cdot \frac{\pi}{2}}}=63 \mathrm{nV} / \sqrt{\mathrm{Hz}} \rightarrow g_{m 1,2}=8.34 \mu \mathrm{S}
$$

The high-pass filter cutoff frequency is set by $\mathrm{C}_{2}$ and the MOSFET-based resistive elements in the feedback path. The incremental resistance of these electronic resistors is over $10^{11} \Omega$ for $|\Delta \mathrm{V}|<0.2 \mathrm{~V}$, where $|\Delta \mathrm{V}|$ is the voltage across each resistive element [4]. This results in a DC baseline stabilization with a cutoff frequency in the 10$100 \mathrm{mHz}$ range. The required $1 \mathrm{kHz}$ signal bandwidth, feedback factor, and $g_{m l, 2}$ of the input differential pair set the first stage differential amplifier output load capacitance, $\mathrm{C}_{\mathrm{L}}$, requirement, as shown in Equation 4-5.

$$
\omega_{-3 d B}=\frac{\omega_{\text {unity-gain }}}{(1 / f)}=\frac{g_{m 1,2}}{(1 / f) C_{L}} \rightarrow C_{L}=\frac{8.34 \mu S}{2 \pi \cdot 1 \mathrm{kHz} \cdot 80}=16.6 \mathrm{pF}
$$

By combining the voltage noise power spectral density expression from Equation 4-3 with an expression of the closed-loop bandwidth as a function of $g_{m 1,2}$, the closedloop gain, and the load capacitance of $\mathrm{C}_{\mathrm{L}}$, the required integrated noise level and closed- 
loop gain can determine the load capacitance directly. Equation 4-6 is an expression for the product of the OTA input-referred voltage noise power spectral density and the square root of the OTA closed-loop bandwidth or, equivalently, the integrated inputreferred thermal noise.

$$
\frac{v_{i N}}{\sqrt{\Delta \mathrm{f}}} \cdot \sqrt{B W}=\sqrt{\frac{16}{3} \frac{k T}{g_{m 1,2}} \frac{3}{2}} \cdot \sqrt{\frac{g_{m 1,2}}{2 \pi(1 / f) C_{L}} \frac{\pi}{2}}=v_{i N}
$$

Simplifying Equation 4-6, the integrated OTA input-referred thermal noise is expressed as a function of the closed-loop feedback factor and the OTA load capacitance in Equation 4-7.

$$
v_{i N}=\sqrt{\frac{4 k T}{2(1 / f) C_{L}}}
$$

The previous design considerations have already determined the OTA inputreferred thermal noise requirement, $v_{i N}=2.5 \mu \mathrm{V}_{R M S}$, and the feedback factor, $1 / f=80$. The required load capacitance can then be calculated as $\mathrm{C}_{\mathrm{L}}=16.6 \mathrm{pF}$. By rearranging Equation 4-5, it can be verified that the same required small-signal transconductance of the input differential pair, $g_{m l, 2}$, is obtained using this approach, as shown in Equation 48.

$$
g_{m 1,2}=1 \mathrm{kHz} \cdot 2 \pi(1 / f) C_{L}=8.34 \mu \mathrm{S}
$$

In order to minimize current consumption, the front-end amplifier is designed to operate in the moderate inversion, or subthreshold region. The subthreshold characteristic current equation, $\mathrm{I}_{\mathrm{S}}$, can be calculated as a function of the transistor aspect ratio W/L by Equation 4-9,

$$
I_{S}=\frac{2 \mu_{p} C_{o x} V_{t h}^{2}}{\kappa} \frac{W}{L}
$$


where $\kappa$ denotes the change in surface potential for a change in $\mathrm{V}_{\mathrm{GB}}$ and is equal to 0.7 , $\mu_{P}$ is $214 \mathrm{~cm}^{2} / \mathrm{Vs}, \mathrm{C}_{\mathrm{OX}}$ is $112 \mathrm{nF} / \mathrm{cm}^{2}$, or equivalently, $1.12 \mathrm{fF} / \mu \mathrm{m}^{2}$, and $\mathrm{V}_{\mathrm{TH}}$ is equal to $\mathrm{kT} / \mathrm{Q}$ of $26 \mathrm{mV}$, which is the thermal voltage.

An inversion coefficient (IC) can be defined as the ratio of the transistor bias current to the characteristic current. IC $>10$ denotes strong inversion, where device small-signal transconductance is proportional to the square root of the bias current. IC $<$ 0.1 denotes weak inversion, where the small-signal transconductance is proportional to the bias current. For $0.1<\mathrm{IC}<10$, the transistor operates in moderate inversion.

Figure 4-3 shows the relationship between IC, the device small-signal transconductance, and the incremental gain in small-signal transconductance per an increase in bias current. The hand calculation slope is based on the traditional equation for small-signal transconductance as a function of current. The simulation slope is based on the SPICE BSIM3 model. The EKV slope is based on a simulation of the EKV MOSFET analytical model, which is specifically designed to be valid in all regions of operation. IC $=1$ is an optimal operating point, where further reducing IC (going deeper into subthreshold) yields less incremental improvement in $g_{m} / I_{\mathrm{D}}$ ratio than for higher values of IC. 


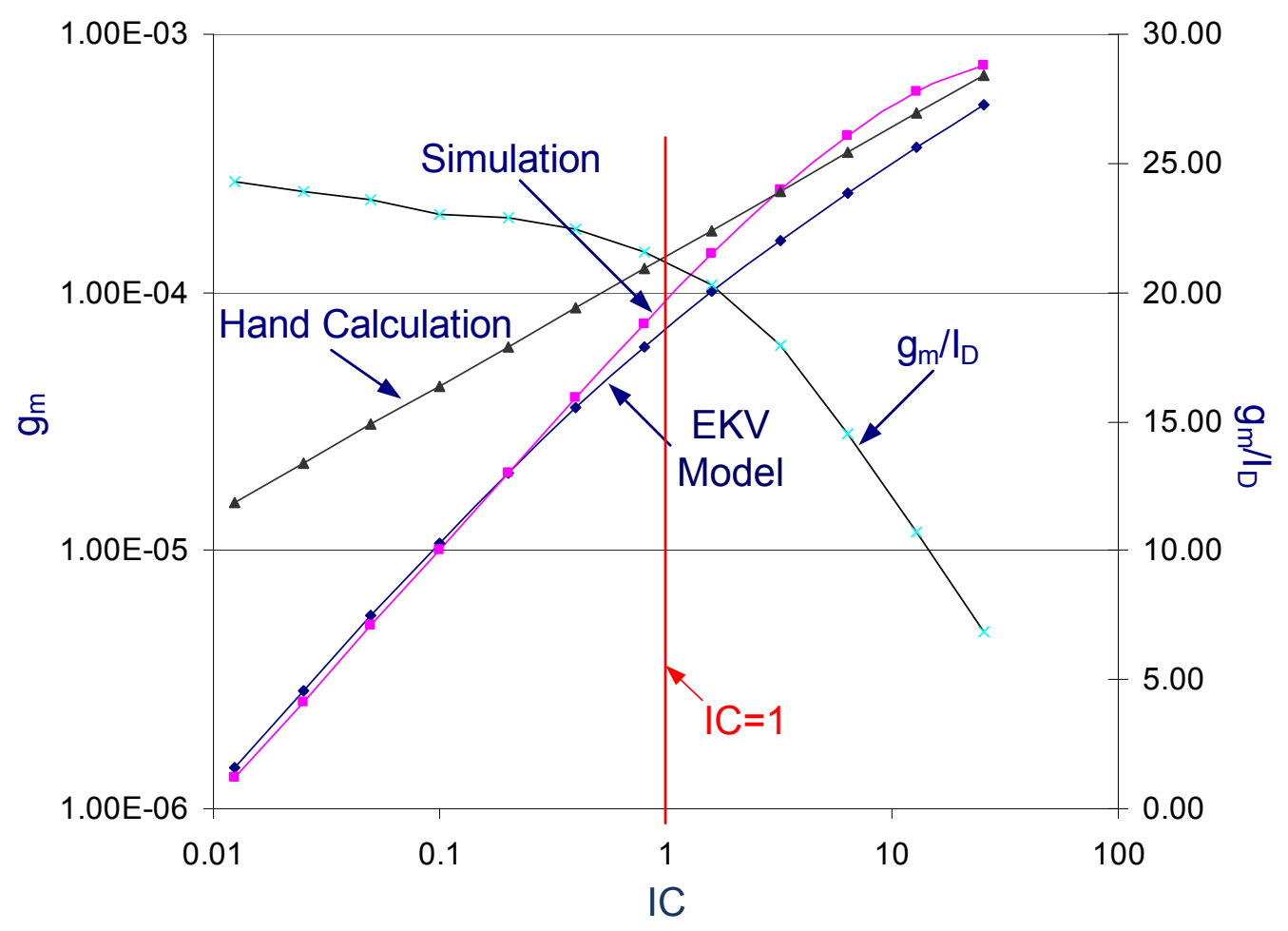

Figure 4-3. Small-Signal Transconductance vs. Inversion Coefficient

Operating at IC $=1$ sets $g_{m} / \mathrm{I}_{\mathrm{D}}=20$. Since the required $g_{m}$ for the input pair is already calculated, the required bias current for each input transistor can be calculated as Equation 4-10.

$$
I_{D}=\frac{g_{m}}{20}=\frac{8.34 \mu \mathrm{S}}{20}=0.42 \mu \mathrm{A}
$$

Operation at $\mathrm{IC}=1$ is achieved when the current equation equals the characteristic current equation. This equality is presented in Equation 4-11.

$$
\frac{1}{2} \mu_{p} C_{o x} \frac{W}{L} V_{D S A T}^{2}=\frac{2 \mu_{p} C_{o x} V_{t h}^{2}}{\kappa} \frac{W}{L}
$$

The $\mathrm{V}_{\text {DSAT }}$ required to achieve this equality can be obtained by rearranging Equation 4-11 to obtain Equation 4-12.

$$
V_{D S A T}=\frac{2 V_{t h}}{\sqrt{\kappa}}=0.065 \mathrm{~V}
$$


Now that $\mathrm{V}_{\text {DSAT }}$ is known, the $\mathrm{W} / \mathrm{L}$ of the input pair can also be calculated for a given $I_{D}$, as shown in Equation 4-13.

$$
\frac{W}{L}=\frac{I_{D}}{\frac{1}{2} \mu_{p} C_{o x} V_{d s a t}^{2}}=8.3
$$

The $\mathrm{W} / \mathrm{L}$ aspect ratio of the input differential pair has now been determined. The $\mathrm{W} \times \mathrm{L}$ is now designed to set the $1 / f$ noise generated by those transistors. The PMOS $1 / f$ noise contribution from the input differential pair can be estimated by using the NLEV=1 equation from HSPICE, as shown in Equation 4-14.

$$
\frac{\overline{v_{N-1 / f, P}}}{\sqrt{\Delta \mathrm{f}}}=\sqrt{2 \cdot \frac{K F_{P} \cdot I_{D S}^{2}}{C_{O X} \cdot W \cdot L \cdot f \cdot g_{m}^{2}}},
$$

where $\overline{v_{N-1 / f, P}} / \sqrt{\Delta f}$ is the input-referred $1 / f$ noise generated by the input differential pair. Substituting in parameters for the given technology $\left(\mathrm{KF}_{\mathrm{P}}=5 \times 10^{-25}\right)$ and design $\left(g_{m}\right.$ $/ \mathrm{I}_{\mathrm{DS}}=20$ ) yields the input-referred $1 / f$ noise as a function of the input transistor gate area, as shown in Equation 4-15.

$$
\frac{\overline{v_{N-1 / f, P}}}{\sqrt{\Delta \mathrm{f}}}=\sqrt{\frac{2 \cdot K F_{P}}{C_{O X} \cdot W \cdot L \cdot f \cdot 400}}=1.5 \sqrt{\frac{1}{W \cdot L \cdot f}} \times 10^{-6} \frac{\mathrm{V}}{\mu \mathrm{m} \sqrt{\mathrm{Hz}}}
$$

The $1 / f$ noise corner frequency is defined as the frequency where the $1 / f$ noise meets the thermal noise floor. A corner frequency target of $25 \mathrm{~Hz}$ is designed in order to be sufficiently below most of the EMG signal's spectral density while not requiring an excessively large transistor geometry. In order to achieve a PMOS $1 / f$ noise corner frequency below $25 \mathrm{~Hz}, \mathrm{~W} x \mathrm{~L}>22 \mu \mathrm{m}^{2}$ is required. Therefore, $\mathrm{W}=27.2 \mu \mathrm{m}$ and $\mathrm{L}=$ $3.2 \mu \mathrm{m}$ are designed for a total area of $87 \mu \mathrm{m}^{2}$. 
The $1 / f$ noise generated by the NMOS current source transistors, $\mathrm{M}_{7,8}$, must also be calculated by Equation 4-16 since they produce a differential-mode output noise, which may be significant compared to the $1 / f$ noise generated by the PMOS input pair.

$$
\frac{\overline{\frac{v_{N-1 / f, N}}{\sqrt{\Delta \mathrm{f}}}}}{\sqrt{\frac{2 \cdot K F_{N} \cdot I_{D S}}{C_{O X} \cdot L^{2} \cdot f \cdot g_{m}^{2}}}}=\sqrt{\frac{2 \cdot K F_{N} \cdot I_{D S}}{C_{O X} \cdot L^{2} \cdot f \cdot\left(2 I_{D S} \mu_{n} C_{O X} W / L\right)}},
$$

where $\overline{v_{N-1 / f, N}} / \sqrt{\Delta f}$ is the $1 / f$ voltage noise power spectral generated by the NMOS current source transistors. Substituting in parameters for the given technology $\left(\mu_{\mathrm{N}}=643\right.$ $\mathrm{cm}^{2} / \mathrm{Vs}$ and $8 \mathrm{KF}_{\mathrm{N}}=2 \times 10^{-28}$ ) and design yields the $1 / f$ noise as a function of the input transistor gate area, as shown in Equation 4-17.

$$
\frac{\overline{v_{N-1 / f, N}}}{\sqrt{\Delta \mathrm{f}}}=\sqrt{\frac{K F_{N}}{C_{O X} \cdot W \cdot L \cdot \mu_{n} C_{O X} \cdot f}}=50 \sqrt{\frac{1}{W \cdot L \cdot f}} \times 10^{-6} \frac{\mathrm{V}}{\mu \mathrm{m} \sqrt{\mathrm{Hz}}}
$$

In order to calculate the $1 / f$ noise corner frequency, the $1 / f$ noise from the current source as a function of device geometry is referred to the differential OTA input as shown in in Equation 4-18.

$$
\frac{\overline{v_{N-1 / f, N}}}{\sqrt{\Delta \mathrm{f}}}=50 \sqrt{\frac{1}{W \cdot L \cdot f}} \frac{g_{m 7,8}}{g_{m 1,2}} \times 10^{-6} \frac{\mathrm{V}}{\mu \mathrm{m} \sqrt{\mathrm{Hz}}}
$$

To achieve a current source $1 / f$ noise corner frequency below $25 \mathrm{~Hz}, \mathrm{~W}$ x L > $6300 \mu \mathrm{m}^{2}$ is required. $\mathrm{V}_{\mathrm{DSAT} 7,8}$ is designed to be $0.25 \mathrm{~V}$ and the bias current is $420 \mathrm{nA}$. This sets the $\mathrm{W} / \mathrm{L}$ ratio for $\mathrm{M}_{7,8}$ to be 0.19 . The gate geometry of $\mathrm{M}_{7,8}$ is designed to be $\mathrm{W}=44.8 \mu \mathrm{m}$ and $\mathrm{L}=230.4 \mu \mathrm{m}$ for a total gate area of $10,322 \mu \mathrm{m}^{2}$.

The low-frequency small-signal open-loop gain of the telescopic OTA, $A_{V, D C}$, is calculated as the small-signal transconductance of the input pair times the OTA smallsignal output resistance, as shown in Equation 4-19. 


$$
A_{V, D C}=\frac{V_{o}^{+}-V_{o}^{-}}{V_{i}^{+}-V_{i}^{-}}=-g_{m 1} \cdot\left(g_{m 3} r_{o 3} r_{o 1}\right) \|\left(g_{m 5} r_{o 5} r_{o 7}\right)
$$

Each $r_{o}$ is calculated as $1 / \mathrm{g}_{\mathrm{ds}}$ as measured from an HSPICE simulation of the amplifier. The calculated low-frequency open-loop small-signal gain is $84.9 \mathrm{~dB}$ and the simulated corresponding gain is $86.9 \mathrm{~dB}$. Table 4-1 presents the OTA design summary.

Table 4-1. OTA Design Summary

\begin{tabular}{|c|c|c|c|c|c|}
\hline Device & Transistor Ratio & $\mathrm{V}_{\mathrm{D}, \mathrm{SAT}}$ & $\mathrm{S}-\mathrm{S} \mathrm{g}_{\mathrm{m}}$ & $\mathrm{S}_{\mathrm{S}} \mathrm{r}_{\mathrm{o}}$ & $\mathrm{I}_{\mathrm{D}}$ \\
\hline $\mathrm{M}_{\text {tail }}$ & $44.8 / 6.4 \mu \mathrm{m}$ & $0.1 \mathrm{~V}$ & $15.7 \mu \mathrm{S}$ & $12.7 \mathrm{M} \Omega$ & $880 \mathrm{nA}$ \\
\hline $\mathrm{M}_{1,2}$ & $27.2 / 3.2 \mu \mathrm{m}$ & $0.065 \mathrm{~V}$ & $8.3 \mu \mathrm{S}$ & $8.78 \mathrm{M} \Omega$ & $440 \mathrm{nA}$ \\
\hline $\mathrm{M}_{3,4}$ & $22.4 / 6.4 \mu \mathrm{m}$ & $0.1 \mathrm{~V}$ & $6.7 \mu \mathrm{S}$ & $47.3 \mathrm{M} \Omega$ & $440 \mathrm{nA}$ \\
\hline $\mathrm{M}_{5,6}$ & $11.2 / 6.9 \mu \mathrm{m}$ & $0.1 \mathrm{~V}$ & $6.1 \mu \mathrm{S}$ & $51.5 \mathrm{M} \Omega$ & $440 \mathrm{nA}$ \\
\hline $\mathrm{M}_{7,8}$ & $44.8 / 230.4 \mu \mathrm{m}$ & $0.25 \mathrm{~V}$ & $2.7 \mu \mathrm{S}$ & $28.1 \mathrm{M} \Omega$ & $440 \mathrm{nA}$ \\
\hline
\end{tabular}

The OTA open-loop frequency response can be calculated and then simulated in HSPICE to confirm the design. The OTA first pole is set by the differential load capacitance and the output resistance of the OTA, as calculated in Equation 4-20.

$$
P_{1}=\frac{1}{2 \pi \cdot C_{L} \cdot R_{\text {Out }}}=\frac{1}{2 \pi \cdot 16.68 \mathrm{pF} \cdot 2.12 \mathrm{G} \Omega}=4.5 \mathrm{~Hz}
$$

The calculated first pole is closely matched to the simulated value of $3.5 \mathrm{~Hz}$. The OTA second pole is set by the small-signal transconductance of $\mathrm{M}_{3,4}$ and the parasitic capacitance between $\mathrm{M}_{1,2}$ and $\mathrm{M}_{3,4}$ as shown in Equations 4-21 and 4-22.

$$
C_{P, 1-4}=C_{g s 3,4}+C_{s b 3,4}+C_{d b 1,2}+C_{g d 1,2}=(53.1+39.5+140+5.3) \mathrm{fF}=238 \mathrm{fF}
$$




$$
P_{2}=\frac{g_{m 3,4}}{2 \pi \cdot\left(C_{P, 1-4}\right)}=\frac{6.72 \mu \mathrm{S}}{2 \pi \cdot 238 \mathrm{fF}}=4.5 \mathrm{MHz}
$$

The simulated second pole location is at $5 \mathrm{MHz}$. The unity-gain frequency is set by the small-signal transconductance of the input differential pair and the differential output load capacitance, as shown in Equation 4-23.

$$
f_{u}=\frac{g_{m 1,2}}{2 \pi \cdot C_{L}}=\frac{8.34 \mu \mathrm{S}}{16.68 \mathrm{pF}}=79.6 \mathrm{kHz}
$$

The phase margin is calculated as Equation 4-24.

$$
P M=90^{\circ}-\arctan \left(\frac{f_{u}}{P_{2}}\right)=89^{\circ}
$$

The simulated unity gain frequency is $77 \mathrm{kHz}$ with $94^{0}$ phase margin. The OTA open-loop HSPICE simulation is shown in Figure 4-4. This simulation is performed with the output common-mode feedback amplifier that is introduced in the next section to set the output DC common-mode level. 


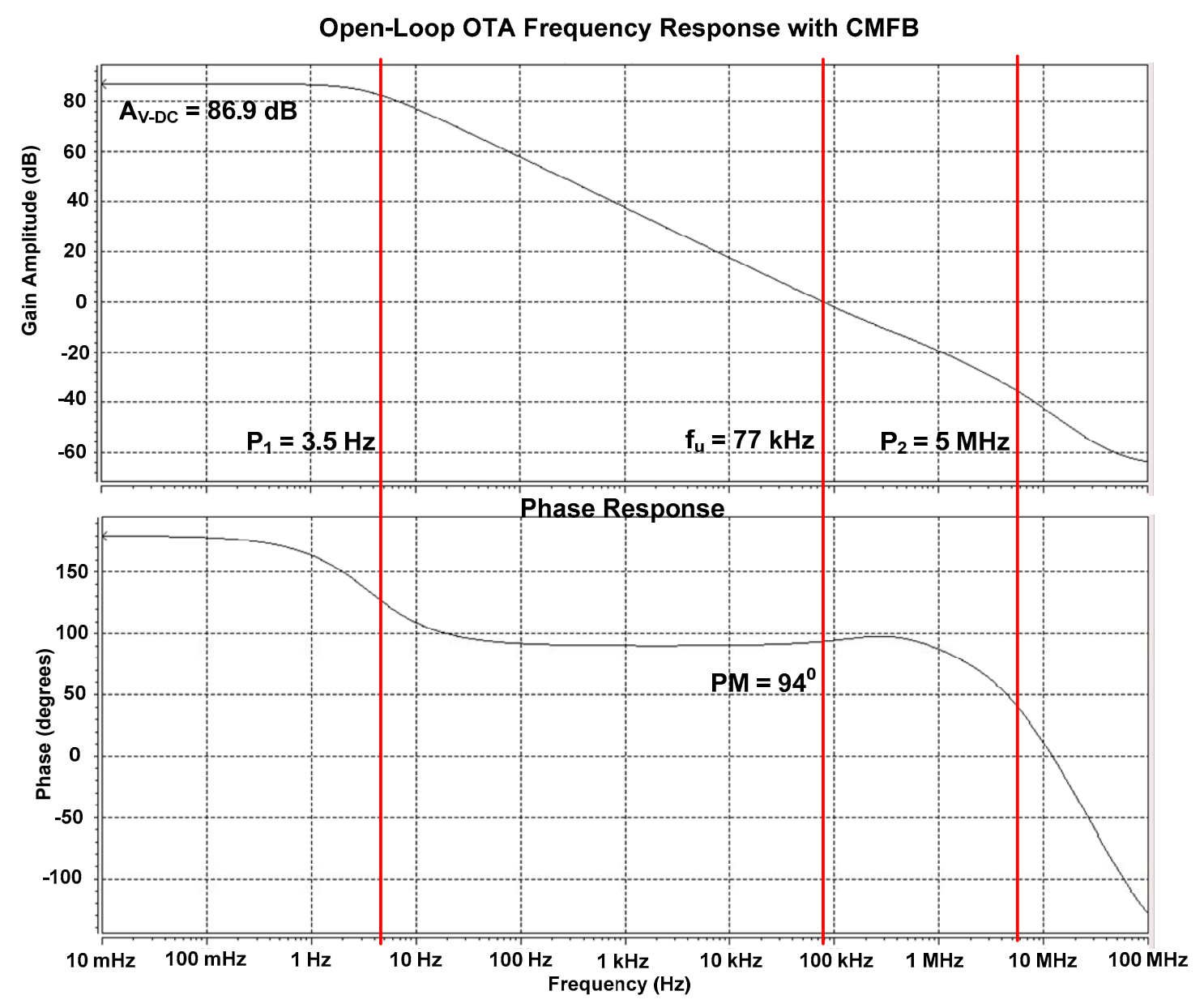

Figure 4-4. Open-Loop OTA Frequency Response Simulation

A closed-loop HSPICE simulation result of the OTA is presented in Figure 4-5.

The high-pass corner frequency is set by the MOSFET-based resistive elements in the feedback path in parallel with the integrating capacitor. The closed-loop corner frequency is set by the unity-gain frequency of the OTA and the feedback factor. 


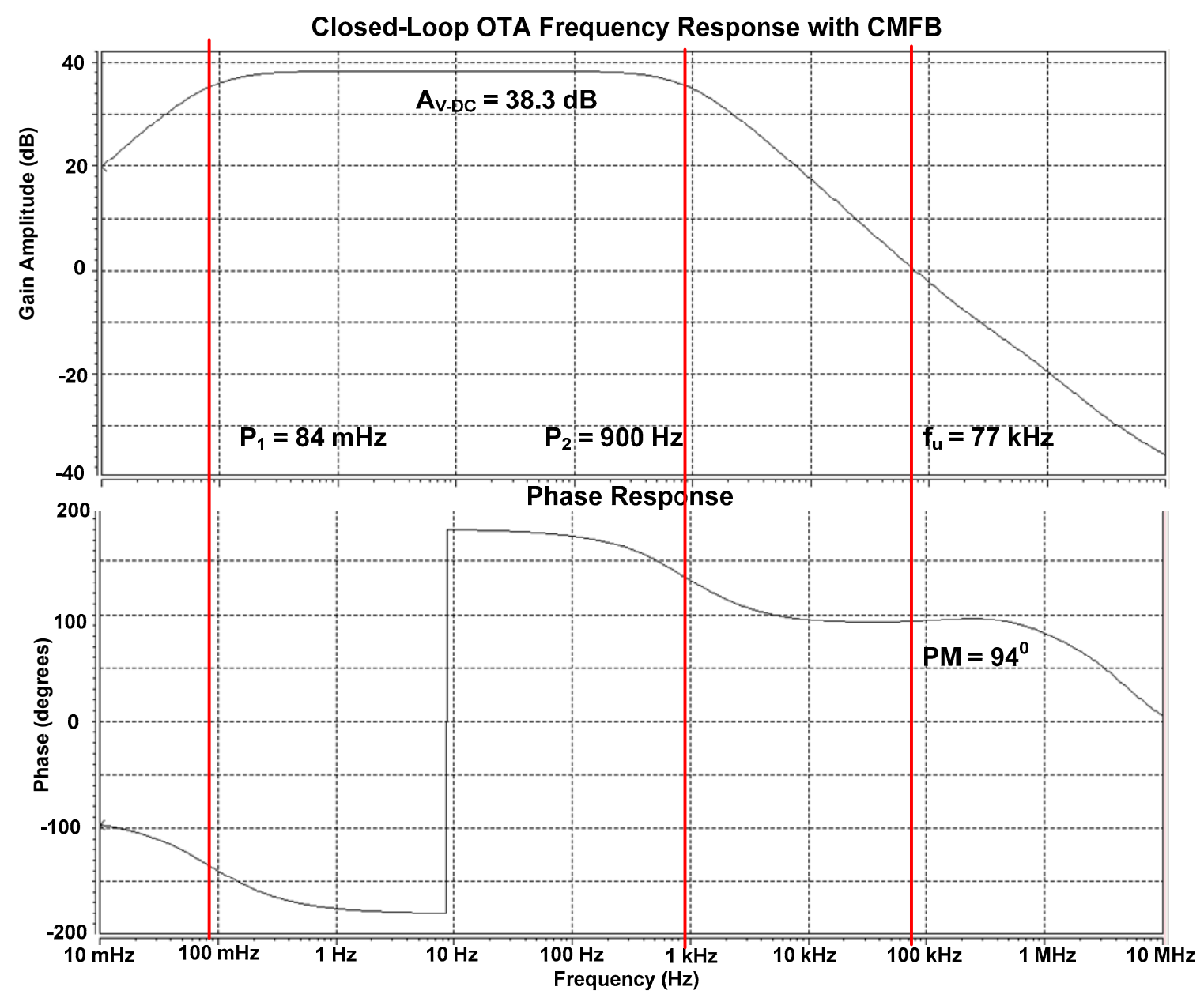

Figure 4-5. Closed-Loop OTA Frequency Response Simulation

\subsection{Continuous-Time Output Common-Mode Feedback Circuit and Amplifier Stability}

A fully-differential amplifier design requires output common-mode feedback (OCMFB) circuitry to set the output common-mode level of the amplifier. A continuoustime OCMFB topology is chosen for this design. Figure 4-6 presents the overall circuit schematics. 


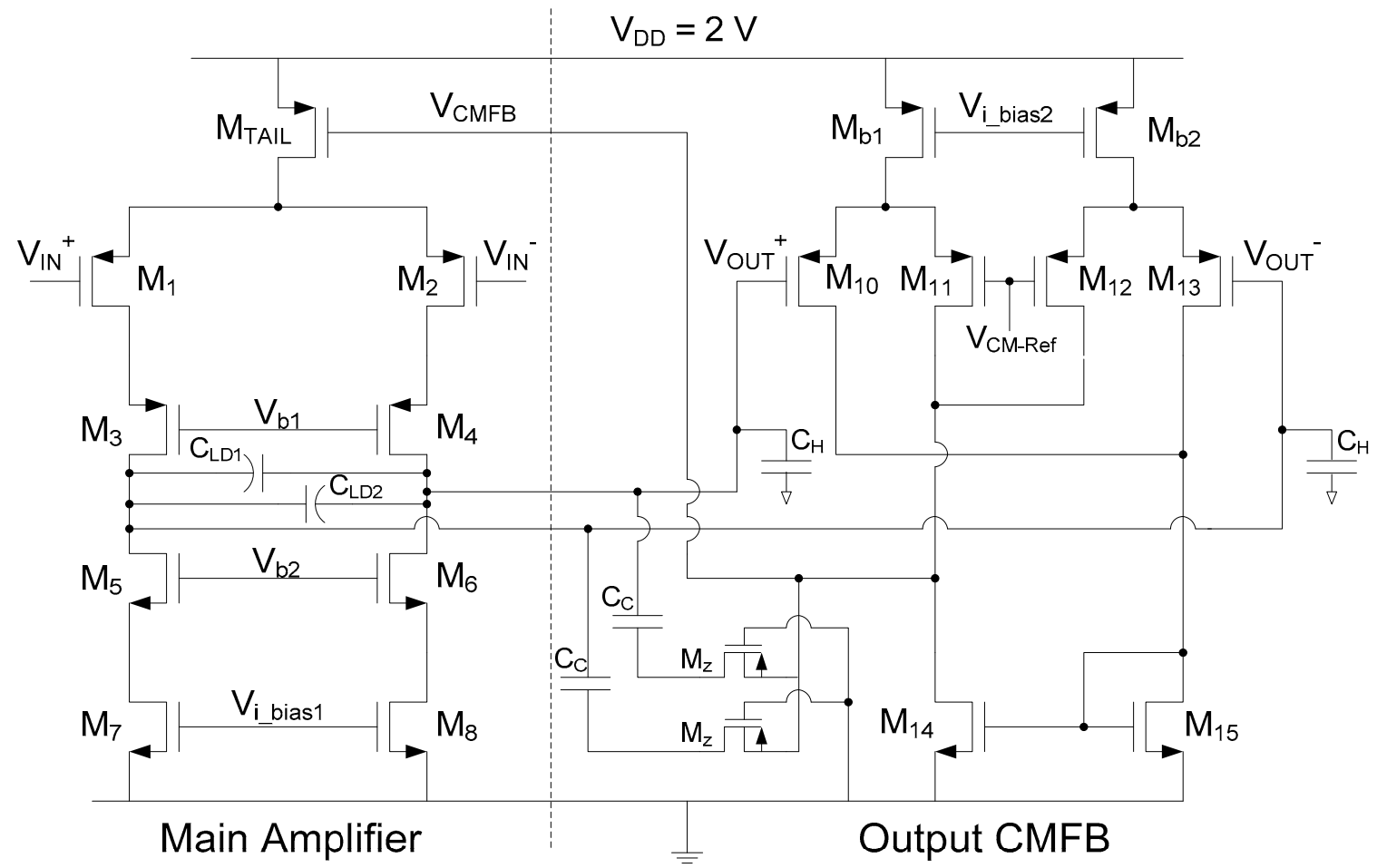

Figure 4-6. Complete OTA Schematic

In continuous-time OCMFB design, the output swing of the OTA is an important consideration, as the OCMFB differential pairs $\mathrm{M}_{10,11}$ and $\mathrm{M}_{12,13}$ must be kept in the active region throughout the full OTA output range. It can be shown that both differential pair transistors can operate in the active region [8] when the magnitude of the differential input voltage is within the range defined in Equation 4-25.

$$
\left|V_{\text {Amplitude, out }}\right| \leq \sqrt{\frac{2 I_{\text {TAIL }}}{k^{\prime}(W / L)}}
$$

Since the bias currents in each differential pair are both equal to $I_{\text {Tail }} / 2$ when the OTA differential output is equal to 0V, Equation 4-25 can be rewritten as Equation 4-26.

$$
\left|V_{\text {Amplitude, out }}\right| \leq \sqrt{2} V_{D S A T}
$$

For an $800 \mathrm{mV}_{\mathrm{PP}}$ differential output swing from the OTA, the OCMFB amplifier must tolerate $\left|\mathrm{V}_{\text {Amplitude,out }}\right|=200 \mathrm{mV}$ zero-to-peak swing on each side. To keep 
differential pairs in the active region, $\mathrm{V}_{\mathrm{DSAT}}$ of the differential pair must be greater than $\left|\mathrm{V}_{\text {Amplitude,out }}\right| / \sqrt{2}=141 \mathrm{mV}$. With this design constraint, a maximum common-mode voltage can be designed to ensure a proper operation, as shown in Equation 4-27,

$$
V_{D D}-V_{D S A T, O C M F B-T a i l}-\left|V_{T, P}\right|-\frac{\left|V_{\text {Amplitude,out }}\right|}{\sqrt{2}}=V_{C M, \text { Max }}+\left|V_{\text {out-peak }}\right|,
$$

where $\mathrm{V}_{\mathrm{DD}}$ is the supply voltage and is equal to $2 \mathrm{~V}, \mathrm{~V}_{\mathrm{DSAT} \text {,Tail }}$ is the $\mathrm{V}_{\mathrm{DSAT}}$ of the tail current transistors $\mathrm{M}_{\mathrm{b} 1, \mathrm{~b} 2}$ and is equal to $0.08 \mathrm{~V}, \mathrm{~V}_{\mathrm{T}, \mathrm{P}}$ is the PMOS threshold voltage and is equal to $0.8 \mathrm{~V}$, and $\mathrm{V}_{\mathrm{CM}, \mathrm{Max}}$ is the maximum supported common-mode voltage. The appropriate common-mode voltage is calculated in Equation 4-28.

$$
V_{C M, M a x}=V_{D D}-V_{D S A T, O C M F B-T a i l}-\left|V_{T, P}\right|-\frac{\left|V_{\text {Amplitude,out }}\right|}{\sqrt{2}}-\left|V_{\text {Amplitude,out }}\right|=0.7 \mathrm{~V}
$$

Setting $\mathrm{V}_{\mathrm{CM}}=0.7 \mathrm{~V}$ results in a swing range of $500 \mathrm{mV}$ to $900 \mathrm{mV}$ on each side of the OTA, which is achievable using a telescopic architecture with PMOS inputs.

The OCMFB current source transistors, $\mathrm{M}_{\mathrm{b} 1,2}$, are each designed to have $880 \mathrm{nA}$ bias current, matching the OTA tail current. This sets the bias current in each differential pair transistor, $\mathrm{M}_{10-13}$, to $440 \mathrm{nA}$. $\mathrm{V}_{\mathrm{DSAT}}$ of the OCMFB differential pair transistors is set to $0.141 \mathrm{~V}$ to accommodate the OTA output swing range. This in turn sets the $\mathrm{W} / \mathrm{L}$ of the OCMFB differential pair transistors to 1.87 and the small-signal transconductance, $g_{m 10-}$ ${ }_{13}$, to $6.24 \mu \mathrm{S}$ The transistors are designed as $11.2 \mu \mathrm{m} / 6.4 \mu \mathrm{m}$ and have a simulated mallsignal transconductance of $4.9 \mu \mathrm{S}$.

The main amplifier and output common-mode feedback (OCMFB) circuit can be modeled as a two-stage amplifier, as shown in Figure 4-7. The stability of this two-stage amplifier must be considered in order to assure a proper operation. The first stage transconductance, $g_{m C M}$, is the small-signal transconductance of the OCMFB amplifier 
and is equal to $g_{m 10-13}$ of $4.9 \mu \mathrm{S}$. The second stage small-signal transconductance, $g_{m T}$, is the common-mode half-circuit transconductance of the OTA from the tail current transistor and is equal to $g_{m T a i l} / 2$ of $7.8 \mu \mathrm{S}$.

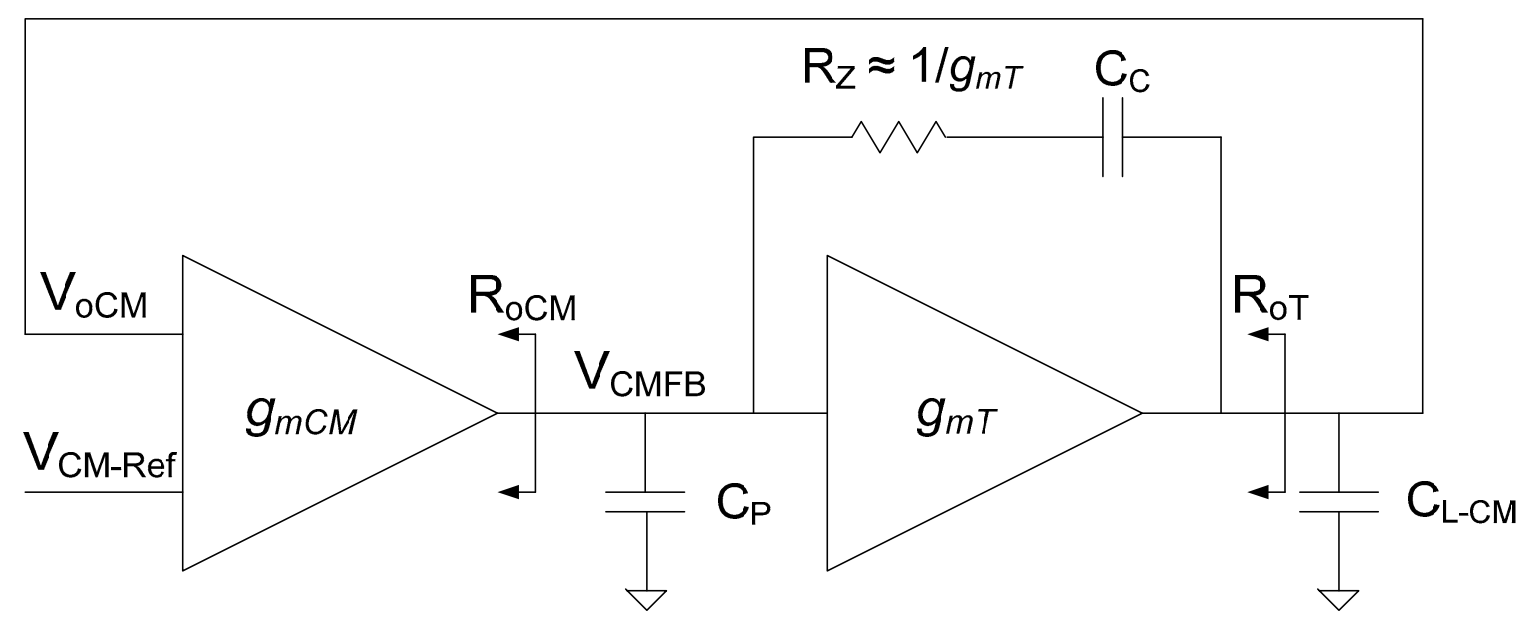

Figure 4-7. OCMFB Model

The compensation capacitor $\mathrm{C}_{\mathrm{C}}$ is designed for minimum size and capacitance value to ensure stability of the two-stage amplifier configuration, where the unity gain frequency is at least three times lower than the second pole. This requires a calculation of the OCMFB system frequency response.

The small-signal output resistance of the first stage, $R_{o C M}$ is calculated as Equation 4-29.

$$
R_{o C M}=r_{014} \|\left(2 r_{o 11} \| 2 r_{o 12}\right)=22.85 \mathrm{M} \Omega
$$

The output resistance of the second stage, $R_{O T}$ is calculated as Equation 4-30.

$$
R_{o T}=g_{m 6} r_{o 6} r_{o 8} \| g_{m 4} r_{o 4} g_{m 2} r_{o 2} r_{\text {oTail }}=8.59 \mathrm{G} \Omega
$$

The DC open-loop gain is calculated as Equation 4-31.

$$
A_{V-D C, C M F B}=\left(g_{m T} R_{o C M}\right) \cdot\left(2 g_{m C M} R_{o T}\right)=143.6 \mathrm{~dB}
$$

The first pole frequency, $\mathrm{P}_{1}$, is set by the combination of the first stage output resistance, $R_{O C M}$, and the second stage $g_{m T} R_{O T}$ product multiplied by compensation 
capacitance, $\mathrm{C}_{\mathrm{C}}$, due to the Miller effect. Even with a low $\mathrm{C}_{\mathrm{C}}$ value in the $\mathrm{pF}$ range, this pole will be less than $1 \mathrm{~Hz}$, as shown in Equation 4-32.

$$
P_{1}=\frac{-1}{2 \pi \cdot R_{o C M}\left(g_{m T} R_{o T} C_{C}\right)}=19 \mathrm{mHz}
$$

The common mode load capacitance can be calculated as Equation 4-33, where $\mathrm{C}_{\mathrm{H}}$ is the input capacitance of the $2.5 \mathrm{x}$ gain stage with a value of $2.5 \mathrm{pF}, \mathrm{C}_{\mathrm{B}_{-} \text {Plate }}$ is the bottom plate of the differential OTA loading capacitance with a value of $150 \mathrm{fF}$, and $\mathrm{C}_{\mathrm{P}}$, out is the parasitic capacitance at the output of the OTA.

$$
C_{L-C M}=C_{H}+C_{B_{-} \text {Plate }}+C_{P, \text { out }}
$$

The parasitic OTA output capacitance can be calculated from the drain capacitances of $\mathrm{M}_{3,4}$ and $\mathrm{M}_{5,6}$ as shown in Equation 4-34.

$$
C_{P, \text { out }}=C_{d b 3,4}+C_{d b 5,6}=2 \cdot 33.9 \mathrm{fF}+2 \cdot 20 \mathrm{fF}=107.8 \mathrm{fF}
$$

Based on these capacitances, the total $\mathrm{C}_{\mathrm{L}-\mathrm{CM}}$ is calculated to be $2.76 \mathrm{pF}$. Additionally, $\mathrm{C}_{\mathrm{P}}$, which is the parasitic capacitance at the output of the OCMFB amplifier, is calculated with Equation 4-35.

$$
\begin{aligned}
& C_{P}=C_{g s, T a i l}+\left(C_{d b}+C_{g d}\right)_{14}+\left(C_{d b}+C_{g d}\right)_{11,12}+\left(C_{g s}+C_{s b}\right)_{Z 1,2} \\
& C_{P}=216 \mathrm{fF}+17.6 \mathrm{fF}+2 \cdot 18.7 \mathrm{fF}+2 \cdot 80 \mathrm{fF}=431 \mathrm{fF}
\end{aligned}
$$

The compensation capacitor $\mathrm{C}_{\mathrm{C}}$ presents small impedance at higher frequencies, so the second pole can be expressed as Equation 4-36.

$$
P_{2}=\frac{-g_{m T}}{2 \pi \cdot\left(C_{L-C M}+C_{P}\right)}=\frac{-7.85 \mu \mathrm{S}}{2 \pi \cdot 3.19 \mathrm{pF}}=392 \mathrm{kHz}
$$

A positive right-hand plane zero resulting from the feed-forward path through the compensation capacitor, set by the compensation capacitance, $\mathrm{C}_{\mathrm{C}}$, and $1 / g_{m T}$ would cause a $+6 \mathrm{~dB} /$ decade increase in gain contribution. This would cause the gain vs. frequency 
response to flatten out along with a phase reduction of 90 degrees, resulting in a stability concern. The zero frequency can be calculated as Equation 4-37.

$$
Z_{1}=\frac{1}{2 \pi \cdot\left(\frac{1}{g_{m T}}\right) C_{C}}=\frac{g_{m T}}{2 \pi \cdot C_{C}}
$$

This zero must be accounted for to ensure a proper phase margin. This can be addressed by placing a nulling resistance, $\mathrm{R}_{\mathrm{Z}}$, in series with the compensation capacitor, $\mathrm{C}_{\mathrm{C}}$, that is approximately equal to $1 / g_{m T}$. A common-gate transistor operating in the triode region satisfies this requirement. The transistors, $\mathrm{M}_{\mathrm{Z1}, 2}$, should be designed with approximately the same size as the tail current transistor (ensuring $R_{Z} \approx 1 / g_{m T}$ ) to push the zero to high frequency so that it does not affect phase margin and stability, as shown in Equation 4-38.

$$
Z_{1}=\frac{1}{2 \pi \cdot\left(\frac{1}{g_{m T}}\right) C_{C}} \rightarrow \frac{1}{2 \pi \cdot\left(\frac{1}{g_{m T}}-R_{Z}\right) C_{C}}
$$

The phase margin is set by the ratio of the unity gain frequency to the second pole, as shown in Equation 4-39.

$$
P M=90-\arctan \left(\frac{f_{u}}{P_{2}}\right)=90-\arctan \left(\frac{g_{m C M}}{g_{m T}} \frac{C_{L-C M}+C_{P}}{C_{C}}\right)
$$

For stability, the unity gain frequency should be at least 3 times lower than the second pole, which would set the phase margin to $72^{\circ}$. Since P2 and gmCM are known at this point, the minimum compensation capacitance to satisfy the stability requirement can be found as shown in Equation 4-40.

$$
C_{C} \geq 3 \frac{g_{m C M}}{g_{m T}}\left(C_{L-C M}+C_{P}\right)=6 \mathrm{pF}
$$


The compensation capacitance was designed to be $5.53 \mathrm{pF}$. This capacitance is implemented as two on-chip capacitors, $\mathrm{C}_{1,2}$, each with a capacitance of $5.53 \mathrm{pF}$, and connected between each OTA differential output and the MOS-based zero-nulling resistance elements, $\mathrm{M}_{\mathrm{Z} 1,2}$.

The unity gain frequency is now set by the small-signal transconductance of the OCMFB amplifier and the compensation capacitance, as shown in Equation 4-41.

$$
f_{u}=\frac{g_{m C M}}{2 \pi \cdot C_{C}}=\frac{4.93 \mu S}{2 \pi \cdot 5.53 \mathrm{pF}}=142 \mathrm{kHz}
$$

The OCMFB design summary is presented in Table 4-2.

Table 4-2. OCMFB Design Summary

\begin{tabular}{|c|c|c|c|c|c|}
\hline Device & Transistor Ratio & $\mathrm{V}_{\mathrm{D}, \mathrm{SAT}}$ & $\mathrm{S}-\mathrm{S} \mathrm{g}_{\mathrm{m}}$ & $\mathrm{S}-\mathrm{S} \mathrm{r}_{\mathrm{o}}$ & $\mathrm{I}_{\mathrm{D}}$ \\
\hline $\mathrm{M}_{\mathrm{b} 1,2}$ & $73.6 / 6.4 \mu \mathrm{m}$ & $0.08 \mathrm{~V}$ & $14.76 \mu \mathrm{S}$ & $13.8 \mathrm{M} \Omega$ & $880 \mathrm{nA}$ \\
\hline $\mathrm{M}_{10-13}$ & $11.2 / 6.4 \mu \mathrm{m}$ & $0.141 \mathrm{~V}$ & $4.93 \mu \mathrm{S}$ & $45.5 \mathrm{M} \Omega$ & $440 \mathrm{nA}$ \\
\hline $\mathrm{M}_{14,15}$ & $4.8 / 4.8 \mu \mathrm{m}$ & $0.15 \mathrm{~V}$ & $8.0 \mu \mathrm{S}$ & $45.9 \mathrm{M} \Omega$ & $880 \mathrm{nA}$ \\
\hline
\end{tabular}

The design of the OCMFB circuit can be verified through SPICE simulation. Using the equations presented above, the hand-calculated frequency response parameters are $\mathrm{P}_{1}=19 \mathrm{mHz}, \mathrm{P}_{2}=392 \mathrm{kHz}, \mathrm{fu}=142 \mathrm{kHz}, \mathrm{PM}=70^{\circ}$, and $\mathrm{A}_{\mathrm{V}, \mathrm{DC}}=143.6 \mathrm{~dB}$. The simulated result is shown below in Figure 4-8. 


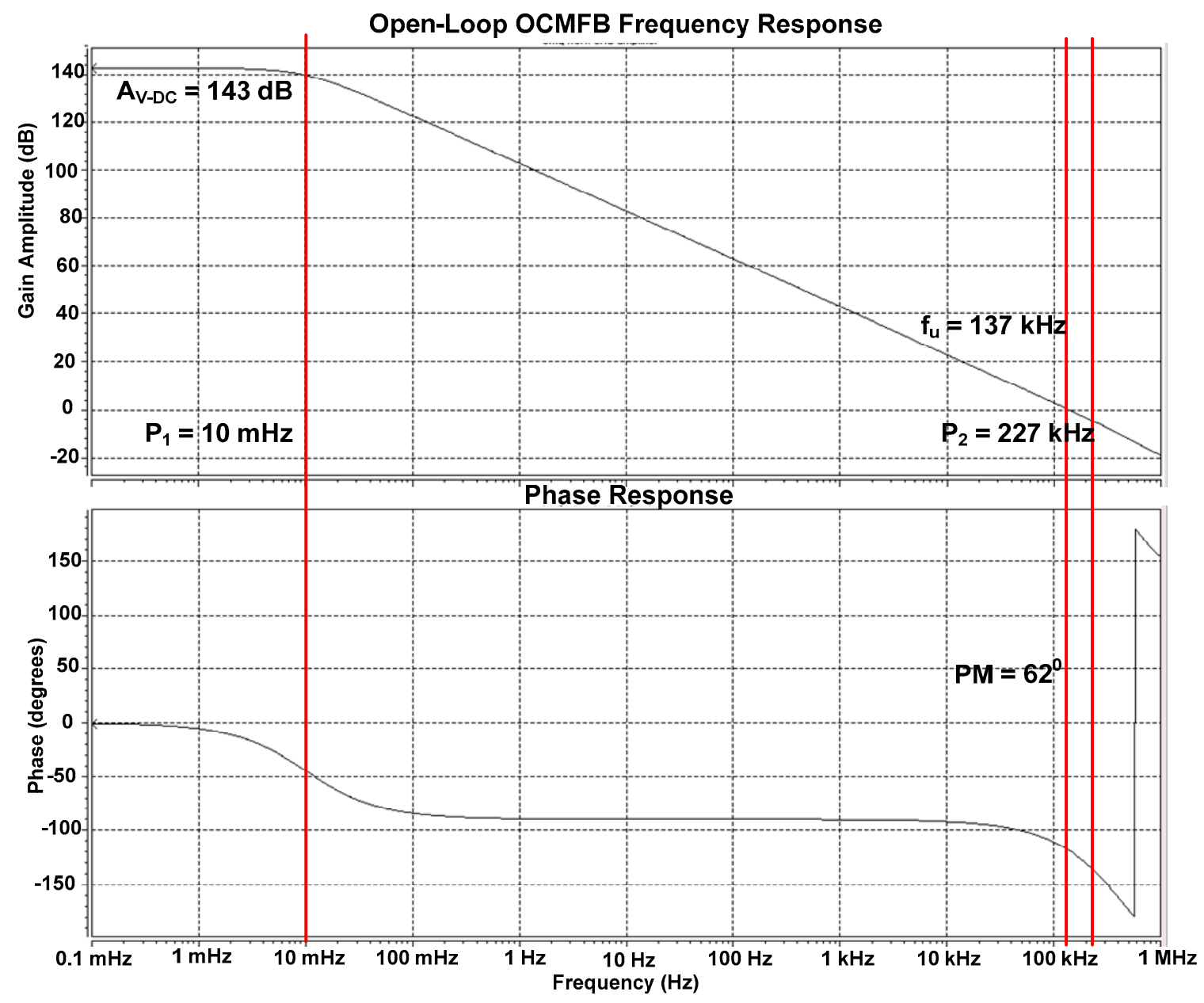

Figure 4-8. Open-Loop OCMFB 2-Stage HSPICE Simulation Result

The simulation matches well with the hand calculations, except the second pole is simulated to be slightly lower than expected, causing a reduced phase margin.

\subsection{Output Common-Mode Feedback Redesign}

After fabrication of the integrated circuit, the design of the OCMFB circuit was revisited. The original OCMFB design approach was to set the bias current of $\mathrm{M}_{10-13}$ equal to that of $\mathrm{M}_{1,2}$, and then to continue the design from there. This was an arbitrary selection and does not yield an optimized result in terms of minimized power 
consumption. An improved design method for the output common-mode feedback circuit is achieved with the following process.

First, set the compensation capacitor, $\mathrm{C}_{\mathrm{C}}$, to be a minimum size of equal to the parasitic capacitance at the output of the first stage of the CMFB amplifier. The parasitic capacitance can be calculated as Equation 4-42 using the previous OCMFB design as a starting point for transistor sizing.

$$
\begin{aligned}
& C_{P}=C_{g s, T a i l}+\left(C_{d b}+C_{g d}\right)_{14}+\left(C_{d b}+C_{g d}\right)_{11,12}+\left(C_{g s}+C_{s b}\right)_{Z 1,2} \\
& C_{P}=216 \mathrm{fF}+17.6 \mathrm{fF}+2 \cdot 18.7 \mathrm{fF}+2 \cdot 80 \mathrm{fF}=431 \mathrm{fF}
\end{aligned}
$$

This sets the compensation capacitance to be $431 \mathrm{fF}$. The second pole of the system is already approximately known since $\mathrm{g}_{\mathrm{mT}}$ is set, and the common-mode capacitance should be similar to previously. The capacitance will change slightly, however; the lower compensation capacitance will require larger differential OTA output capacitors to keep the same OTA $-3 \mathrm{~dB}$ corner frequency. The parasitic will also change slightly due to the redesign of the OCMFB itself. So, assume that $\mathrm{P}_{2}$ is slightly lower at $380 \mathrm{kHz}$ and design the unity-gain frequency to meet the phase margin requirement as shown in Equation 4-43.

$$
\omega_{u, C M} \leq \frac{P_{2}}{3} \rightarrow g_{m, C M}=\frac{C_{C} P_{2}}{3}=343 n S
$$

The minimum required $\mathrm{V}_{\mathrm{DSAT}}$ of the OCMFB input differential pairs, $\mathrm{M}_{10-13}$, of $0.141 \mathrm{~V}$, along with the designed small-signal transconductance sets the required bias current, as shown in Equation 4-44.

$$
I_{D}=\frac{1}{2} g_{m, C M} V_{D S A T}=24 \mathrm{nA}
$$


The gate geometry of $\mathrm{M}_{10-13}$ is now set to 0.1 . The other device gate sizes are set to have similar $\mathrm{V}_{\mathrm{DSAT}}$ values as the previous OCMFB circuit, given the new bias current. The updated design and simulated device parameters are presented in Table 4-3.

Table 4-3. Revised OCMFB Design

\begin{tabular}{|c|c|c|c|c|c|}
\hline Device & Transistor Ratio & $\mathrm{V}_{\mathrm{D}, \mathrm{SAT}}$ & $\mathrm{S}-\mathrm{S} \mathrm{g}_{\mathrm{m}}$ & $\mathrm{S} \mathrm{S}_{\mathrm{o}}$ & $\mathrm{I}_{\mathrm{D}}$ \\
\hline $\mathrm{M}_{\mathrm{b} 1,2}$ & $8 / 12.8 \mu \mathrm{m}$ & $0.09 \mathrm{~V}$ & $800 \mathrm{nS}$ & $110 \mathrm{M} \Omega$ & $46 \mathrm{nA}$ \\
\hline $\mathrm{M}_{10-13}$ & $3.2 / 32 \mu \mathrm{m}$ & $0.185 \mathrm{~V}$ & $200 \mathrm{nS}$ & $3.5 \mathrm{G} \Omega$ & $23 \mathrm{nA}$ \\
\hline $\mathrm{M}_{14,15}$ & $3.2 / 54.4 \mu \mathrm{m}$ & $0.178 \mathrm{~V}$ & $370 \mathrm{nS}$ & $3.3 \mathrm{G} \Omega$ & $46 \mathrm{nA}$ \\
\hline
\end{tabular}

The design of the OCMFB circuit can be verified through SPICE simulation. Using the equations presented above, the hand-calculated frequency response parameters are $\mathrm{P}_{1}=4.8 \mathrm{mHz}, \mathrm{P}_{2}=386 \mathrm{kHz}, \mathrm{fu}=73 \mathrm{kHz}, \mathrm{PM}=79^{\circ}$, and $\mathrm{A}_{V, D C}=149.7 \mathrm{~dB}$. The simulated result is shown below in Figure 4-9. As with the original OCMFB design the simulation matches well with the hand calculations, except the second pole is simulated to be slightly lower than expected, causing a reduced phase margin.

This design has a number of advantages over the originally implemented one. First of all, the bias current is about 20x lower than the original design. By reducing the size of the compensation capacitor, the rest of the differential load capacitance can be achieved by using true differential capacitors instead, which offer a $4 \mathrm{x}$ chip area reduction for the same differential capacitance presented to the OTA. The total capacitors area reduction is approximately $50 \%$ lower than the original design based on decreased $\mathrm{C}_{\mathrm{C}}$ size and increased $\mathrm{C}_{\mathrm{Ld}}$ size. 


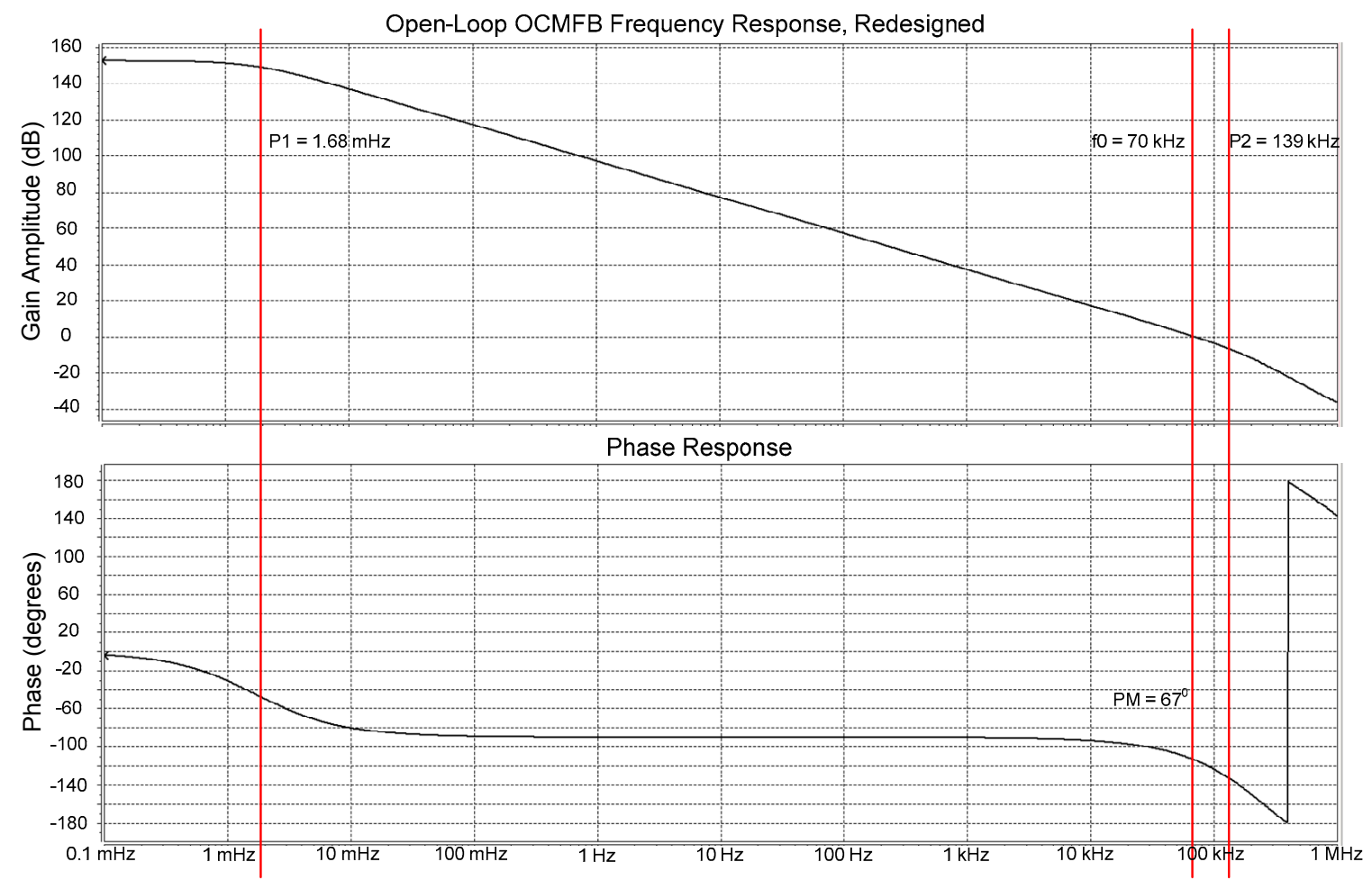

Figure 4-9. Redesigned OCMFB Frequency Response

\subsection{Summary}

The design of the EMG front-end interface amplifier has been presented in this chapter. The amplifier is AC-coupled, fully-differential, and has closed-loop voltage gain set by on-chip capacitance ratio. The amplifier gain is designed to properly interface the EMG signal range to the input of the $2.5 \mathrm{x}$ gain stage and $\mathrm{ADC}$ full range. The $\mathrm{DC}$ baseline stabilization is provided by MOSFET-based resistive elements in the feedback path exhibiting very high incremental resistance. The amplifier core is a differential continuous-time telescopic operational transconductance amplifier (OTA) designed to operate in the moderate inversion region. Amplifier noise is carefully considered and designed to be comparable to the minimum detectable EMG signal. A continuous-time output common mode feedback circuit is implemented to set the amplifier's common- 
mode level appropriately to accommodate the full OTA output signal swing as well as to properly interface with the $2.5 \mathrm{x}$ gain stage. An improved re-design approach to the OCMFB circuit is also presented. 


\section{Chapter 5}

\section{Remote RF Powering and Data Telemetry System}

\subsection{Overview}

The EMG sensing electronics are powered by a wireless RF powering system built around inductively coupled resonant coils. The primary (external) circuit is a series resonant tank driven by a high-efficiency Class-E power amplifier [7][9]. The secondary (implantable) circuit is weakly coupled to the primary coil and consists of a parallel resonant tank interfaced with on-chip integrated $\mathrm{RF}$ to $\mathrm{DC}$ rectification and regulation electronics. Both circuits are tuned to the same operating resonant frequency $\omega_{0}$ of 8 MHz. The inductor coils for the primary and secondary circuits are generally aligned coaxially and are designed to be separated by 1 to $2 \mathrm{~cm}$, a typical thickness of the skin above target muscles in a residual lower limb. The system will still function with a reduced wireless power transfer efficiency if the separation distance is somewhat larger than $2 \mathrm{~cm}$. For separation distances much larger than $2 \mathrm{~cm}$, a larger secondary coil design should be considered. The inductive coupling efficiency is determined in large part by the coupling factor, $k$, which is a function of coils geometry and separation distance. This chapter presents the analysis and design of the inductively coupled architecture. 


\subsection{Analysis of Inductively Coupled Circuit}

The inductive RF powering system can be analyzed as a weakly coupled air-core transformer. In an iron-core transformer, the magnetic flux generated by the alternating current in the primary coil is transferred to the secondary coil with a coupling factor, $k$, near unity. In a wireless power transmission system, the link is determined by the mutual inductance $M=k \sqrt{L_{1} L_{2}}$. The coupling factor, $k$, is a critical parameter in the wireless power transmission system. It is much smaller than unity in an air-core design and is a function of coil geometry. Although there are a number of closed-form approximations for various coil geometries [10], the coupling factor should be verified experimentally.

In a generalized inductively coupled circuit, an input voltage, $\mathrm{V}_{\mathrm{ac} \_ \text {in }}$, drives a series resonant circuit composed of a series resistance, $\mathrm{R}_{1}$, a resonating capacitance, $\mathrm{C}_{1}$, and the primary inductor coil, $\mathrm{L}_{1}$. Energy is transferred to the secondary, parallel resonant tank by the mutual inductance $M$. The secondary tank is composed of the secondary coil, $\mathrm{L}_{2}$, the series resistance of the secondary coil, $\mathrm{R}_{2}$, and a parallel resonating capacitor, $\mathrm{C}_{2}$. $\mathrm{R}_{\mathrm{ac} \_ \text {load }}$ is the total AC resistive load presented to the secondary tank and $\mathrm{V}_{\mathrm{ac} \_ \text {_out }}$ is the $\mathrm{AC}$ voltage developed over $\mathrm{R}_{\mathrm{ac} \_ \text {load. }}$. Both the primary and secondary tanks are tuned to the same operating frequency, $\omega_{0}=2 \pi f_{0}=1 / \sqrt{L_{1} C_{1}}=1 / \sqrt{L_{2} C_{2}}$, for an optimal performance. 


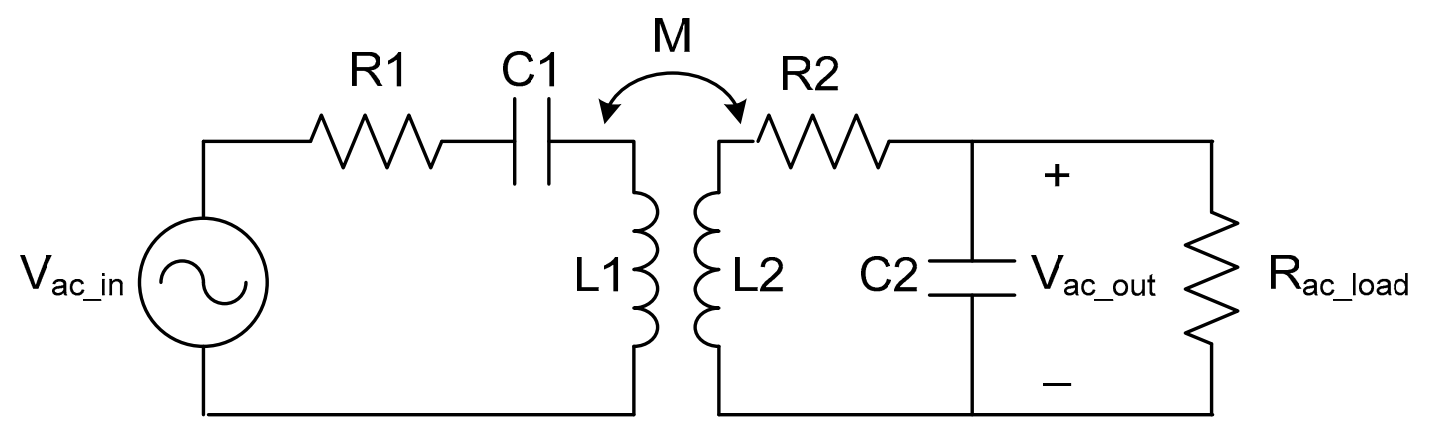

Figure 5-1. Simplified RF Powering Schematic

It can be shown [11] that the voltage gain from $\mathrm{V}_{\mathrm{ac} \_ \text {in }}$ to $\mathrm{V}_{\mathrm{ac} \_ \text {out }}$ can be as shown in Equation 5-1 once $k$, and therefore $M$, is known for a particular coil geometry,

$$
A_{V}=\frac{V_{\text {out }}}{V_{\text {in }}}=\frac{\omega^{2} M L_{2}}{(\omega M)^{2}+R_{1} R_{2}+R_{1} \frac{\left(\omega L_{2}\right)^{2}}{R_{\text {AC-load }}}},
$$

where $\omega$ is the operating frequency and is equal to $2 \pi \times 8 \mathrm{MHz}, \mathrm{M}$ is the mutual inductance of $103 \mathrm{nH}, \mathrm{L}_{2}$ is equal to $2.00 \mu \mathrm{H}, \mathrm{R} 1$ is equal to $2.15 \Omega, \mathrm{R}_{2}$ is equal to 13.65 $\Omega$, and $\mathrm{R}_{\mathrm{AC}-\text { load }}$ is equal to $15 \mathrm{k} \Omega$.

In order to optimize power transfer efficiency from the primary to secondary coil, the voltage gain must be maximized for a given $\mathrm{R}_{\mathrm{AC}-\text { load. }}$ To do this, the mutual inductance, $M$, is enhanced by increasing $k, \mathrm{~L}_{1}$, and $\mathrm{L}_{2}$ while minimizing losses in the circuit through using inductor coils with low-loss winding and self-resonant frequencies much higher than the operating frequency. The physical dimensions of the internal and external coils are set by geometric restrictions as described in Section 5.3. The objective is to optimize the number of turns of the coils within the design constraints to achieve the best power transfer efficiency.

It is important to accurately measure the coupling factor $k$. The procedure for measuring $k$ is to first characterize each inductor coil using a precision impedance 
analyzer over a frequency range of interest, for example from $1 \mathrm{MHz}$ to $10 \mathrm{MHz}$. The primary and secondary circuits are then tuned to the same operating frequency $\omega_{0}$. The primary circuit is driven by an $\mathrm{AC}$ voltage with a known amplitude at $\omega_{0}$, and the amplitude of the output signal is measured with an oscilloscope with the coils separated by a known and fixed distance $(1 \mathrm{~cm}$ in these tests). With the measured voltage gain, the coupling factor, $k$, can be determined using Equation 5-1 and each component's impedance values at the operating frequency. In order to verify that the coupling factor is independent of frequency, this experiment is repeated for several operating frequencies.

Several candidate external inductor coils are characterized and their coupling factors with respect to a reference 6-mm diameter, 30-turn secondary coil are measured. The coil optimization and selection process for the Class is presented in the next section. For the final system design, a properly-tuned Class-E power amplifier architecture is employed to achieve a high efficiency, as shown in Figure 5-2.

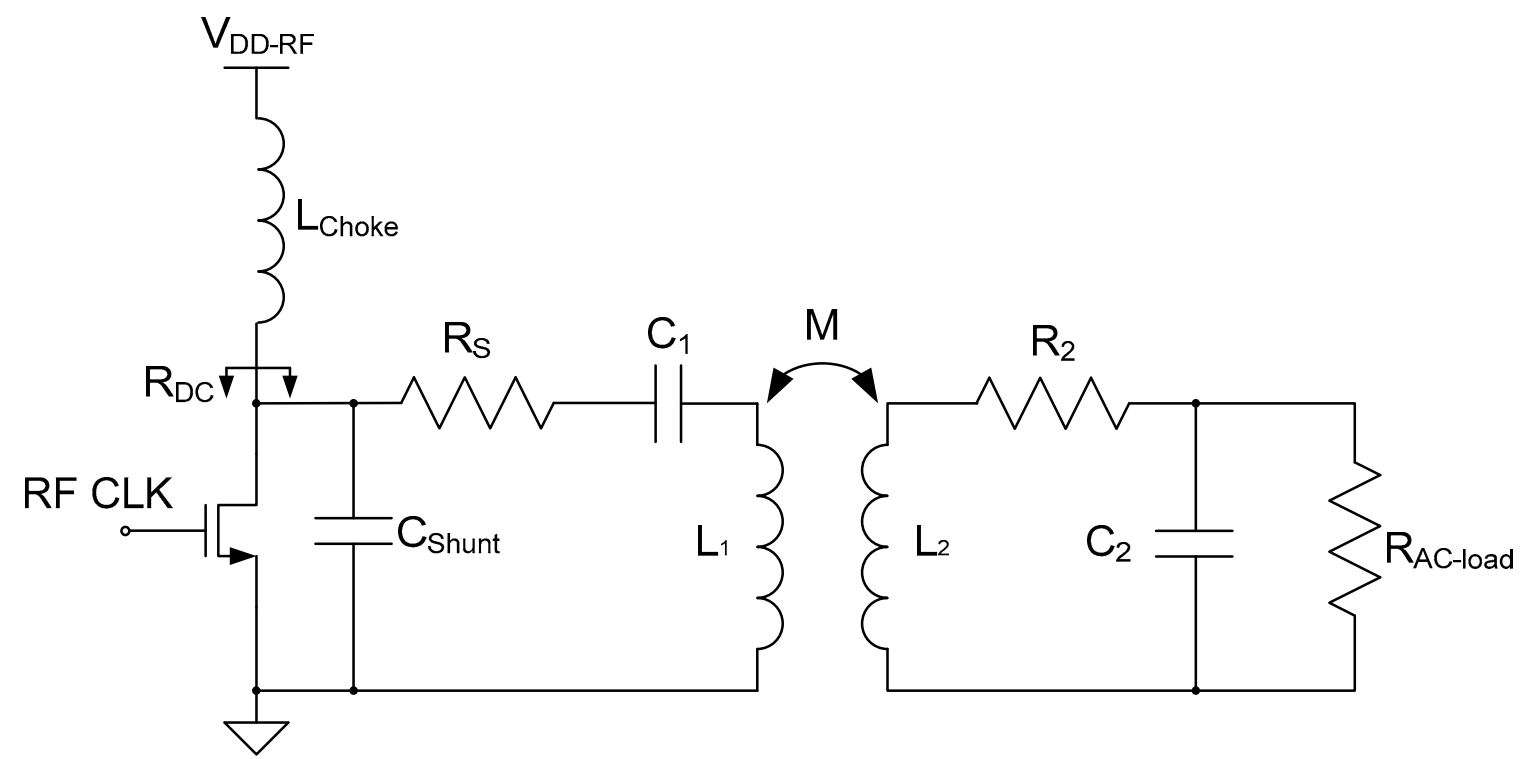

Figure 5-2. Class-E Amplifier for RF Powering 
The Class-E tuning process begins with the operating point equations [7] and experimentally fine-tuning the shunt and series resonating capacitors, until a classic Class-E waveform is obtained at the drain of the switching MOSFET [9][12]. The choke inductor, $\mathrm{L}_{\text {Choke, }}$, is selected to be sufficiently large that the input current ripple is sufficiently low to be considered DC. The inductor value of $\mathrm{L}_{1}$ is set by the geometry of the external coil. The total series resistance of the primary tank, $R_{S}$, can be designed as necessary to set the quality factor, $\mathrm{Q}$, of the primary tank. It is desirable to have control of this parameter for the case of using a passive phase shift keying (PSK) data telemetry system, such as the one implemented in this design and described later in this chapter. If the primary tank Q is too high to support the PSK data bandwidth it may be lowered by adding extra resistance in series with $R_{1}$ for a total of $R_{S}$.

The Class-E amplifier is tuned by proper selection of the primary series capacitance, $\mathrm{C}_{1}$, and a shunt capacitance across the switching FET, $\mathrm{C}_{\text {Shunt }}$. The design equations for these capacitors are shown in Equations 5-2 and 5-3.

$$
\begin{aligned}
& C_{1}=\left[\omega^{2} L_{1}-\frac{\pi}{4}\left(\frac{\pi^{2}}{4}-1\right) \omega R_{S}\right]^{-1} \\
& C_{\text {Shunt }}=\frac{8}{\pi\left(4+\pi^{2}\right) \omega R_{S}}
\end{aligned}
$$

When the system is tuned properly, this waveform will have a $50 \%$ duty cycle with a minimal over- or under-shoot. The RF transistor ideally switches when the drain voltage is $0 \mathrm{~V}$ and the derivative of the drain voltage waveform is also 0 , enabling high-efficiency operation.

\subsection{Design Optimization of RF Power Coils}


The design of the RF powering coils is constrained by geometry, but may be optimized for wireless power transfer efficiency. The implantable secondary coil must interface with the EMG sensing electrodes and electronics. Therefore, a coil outer diameter of $6 \mathrm{~mm}$ is selected. The operating frequency range is limited by the selfresonant frequency (SRF) of the coil due to parasitic capacitance of the coil. Above the SRF, the coil becomes capacitive and is therefore non-functional for this application. As the operating frequency approaches the SRF, the system performance quickly degrades as the coil equivalent series resistance increases dramatically. The SRF is related to the geometry of the coil, especially the number of turns. A secondary coil of approximately 30 turns exhibiting a SRF of $45 \mathrm{MHz}$ is chosen as the internal coil to characterize the wireless link. The coil has inductance value of $6.9 \mu \mathrm{H}$ and is made of 36-gage enameled wire held together with two-part epoxy mixed in a 1:1 ratio and allowed to air-cure.

The external primary coil is designed such that its diameter is small compared to the prosthetic limb socket circumference for an optimal planar alignment. To meet this requirement, a maximum outer diameter of $2 \mathrm{~cm}$ is specified. A spiral coil design is selected due to its flat configuration for easy interfacing with the prosthetic socket and comparable coupling efficiency when compared to a solenoidal coil of similar dimensions and number of turns. As with the secondary coil, the SRF of the primary coil must be much higher than the operating frequency so that it appears inductive at the operating frequency.

An optimal number of turns for the primary coil can be found to maximize power transfer to the implant coil. In order to obtain a high efficiency, a class-E amplifier is 
used to characterize the wireless link. To derive a power transfer efficiency equation, the power efficiency of a linear system, as shown in Equation 5-4, is recalled.

$$
\eta_{\text {eff }}=\frac{P_{\text {out }}}{P_{\text {in }}}=\frac{V_{\text {ac-out }}^{2}}{V_{a c-\text { in }}^{2}} \frac{R_{1}}{R_{A C \text {-load }}}=\left(A_{V}\right)^{2} \frac{R_{1}}{R_{A C-\text { load }}}
$$

This equation can be extended for use with the class-E amplifier by acknowledging that the class-E amplifier does not have $100 \%$ power efficiency from the DC supply to the RF energy in the tank, as shown in Equation 5-5. A detailed analysis of the Class-E power efficiency for this application can be found in [13].

$$
\eta_{\text {eff_DC }} \cong\left(A_{V}\right)^{2} \times\left(\frac{R_{1}}{R_{A C \text {-load }}}\right) \times \eta_{\text {class }-E}
$$

Equation 5-5 can be rewritten as Equation 5-6 by substituting in the voltage gain expression from Equation 5-1.

$$
\eta_{\text {eff }} \approx\left(\frac{\omega^{2} M L_{2}}{R_{1} R_{2}+(\omega M)^{2}+R_{1} \frac{\left(\omega L_{2}\right)^{2}}{R_{A C-\text { load }}}}\right)^{2} \frac{R_{1}}{R_{A C-\text { load }}} \eta_{\text {Class-E }}=\frac{\frac{V_{\text {out }}^{2}}{R_{A C-\text { load }}}}{V_{D D-R F} \cdot I_{D D-R F}} \eta_{\text {Class-E }}
$$

A summary of the coils built and tested to optimize power transfer efficiency is presented in Table 5-1and a plot of predicted and measured power transfer efficiency from $\mathrm{DC}$ power dissipation of the Class-E amplifier to the power delivered into the $\mathrm{R}_{\mathrm{AC}}$ load when using a Class-E amplifier is presented in Figure 5-3. 
Table 5-1. RF Coil Summary

\begin{tabular}{|c|c|c|c|c|}
\hline Coil Type & $\begin{array}{c}\text { Self-Inductance } \\
@ 8 \mathrm{MHz}\end{array}$ & $\begin{array}{c}\text { Series Resistance } \\
\text { @ } 8 \mathrm{MHz}\end{array}$ & $\begin{array}{c}\text { Self-Resonant } \\
\text { Frequency }\end{array}$ & $\begin{array}{c}\text { Coupling Factor } \\
k @ 1 \mathrm{~cm}\end{array}$ \\
\hline $\begin{array}{c}\text { Spiral } \\
\text { 6-Turn }\end{array}$ & $680 \mathrm{nH}$ & 0.16 & $>110 \mathrm{MHz}$ & 0.02546 \\
\hline $\begin{array}{c}\text { Spiral } \\
\text { 10-Turn }\end{array}$ & $2.0 \mu \mathrm{H}$ & 0.85 & $65 \mathrm{MHz}$ & 0.02765 \\
\hline $\begin{array}{c}\text { Solenoid } \\
\text { 10-Turn }\end{array}$ & $2.63 \mu \mathrm{H}$ & 0.52 & $66 \mathrm{MHz}$ & 0.02019 \\
\hline $\begin{array}{c}\text { Solenoid 10- } \\
\text { Turn, 2-Layer }\end{array}$ & $3.0 \mu \mathrm{H}$ & 1.4 & $47 \mathrm{MHz}$ & 0.02145 \\
\hline $\begin{array}{c}\text { Solenoid 14- } \\
\text { Turn, 2-Layer }\end{array}$ & $5.38 \mu \mathrm{H}$ & 5.8 & $32 \mathrm{MHz}$ & 0.02153 \\
\hline $\begin{array}{c}\text { Secondary } \\
\text { 30-Turn }\end{array}$ & $6.95 \mu \mathrm{H}$ & 13.65 & $45 \mathrm{MHz}$ & N/A \\
\hline
\end{tabular}

Based on the predicted power transfer efficiency curves shown in Figure 5-3, the system is tuned to several operating points (choice of coil and operating frequency) in order to verify the predicted efficiencies. An $\mathrm{R}_{\mathrm{AC} \text {-Load }}$ of $1.95 \mathrm{k} \Omega$ is assumed for this test. Based on the testing, a 2-cm diameter, 10-turn spiral coil and an operating frequency of 8 $\mathrm{MHz}$ are chosen for an optimal power transfer efficiency. 


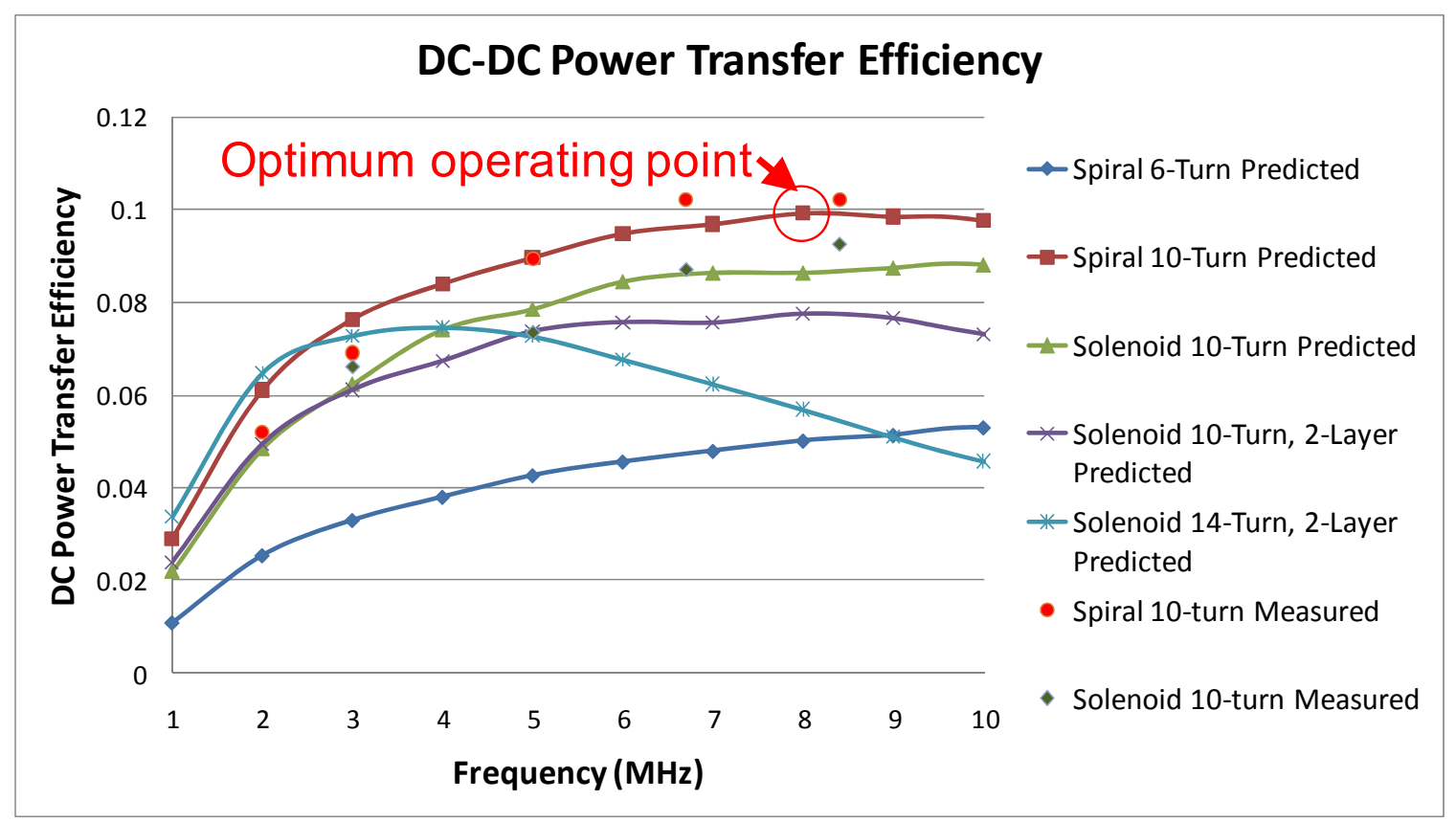

Figure 5-3. Power Transfer Efficiency $\left(\mathbf{R}_{\mathrm{AC}-\mathrm{Load}}=1.95 \mathrm{k} \Omega\right)$

\section{$5.4 \boldsymbol{R}_{A C-l o a d}$ Derivation}

The voltage rectifier on the secondary circuit is designed to be a voltage doubler, as shown in Figure 5-4. This design produces two supply voltages, $V_{D C_{-} \text {low }}$ and $V_{D C_{-} \text {high }}$. In one cycle, $\mathrm{C}_{\text {filterl }}$ is charged to $V_{D C_{-} \text {low }}$ of $2.5 \mathrm{~V}$ through diode-connected NMOS device $\mathrm{M}_{\mathrm{d} 1}$, which has a corresponding diode voltage drop of $V_{\text {diode_low }}$, equal to $0.75 \mathrm{~V}$. In the other cycle of operation, $\mathrm{C}_{\text {fliter2 }}$ is also charged to approximately $V_{D C \_l o w}$ through $\mathrm{M}_{\mathrm{d} 2}$. The voltage across both capacitors $\mathrm{C}_{\text {filter1 }}$ and $\mathrm{C}_{\text {filter2 }}$ produces $V_{D C \_ \text {high }}$, which is equal to $4.5 \mathrm{~V}$ and has a total combined diode voltage drop of $V_{\text {diode }}$ high of $1.25 \mathrm{~V}$. 


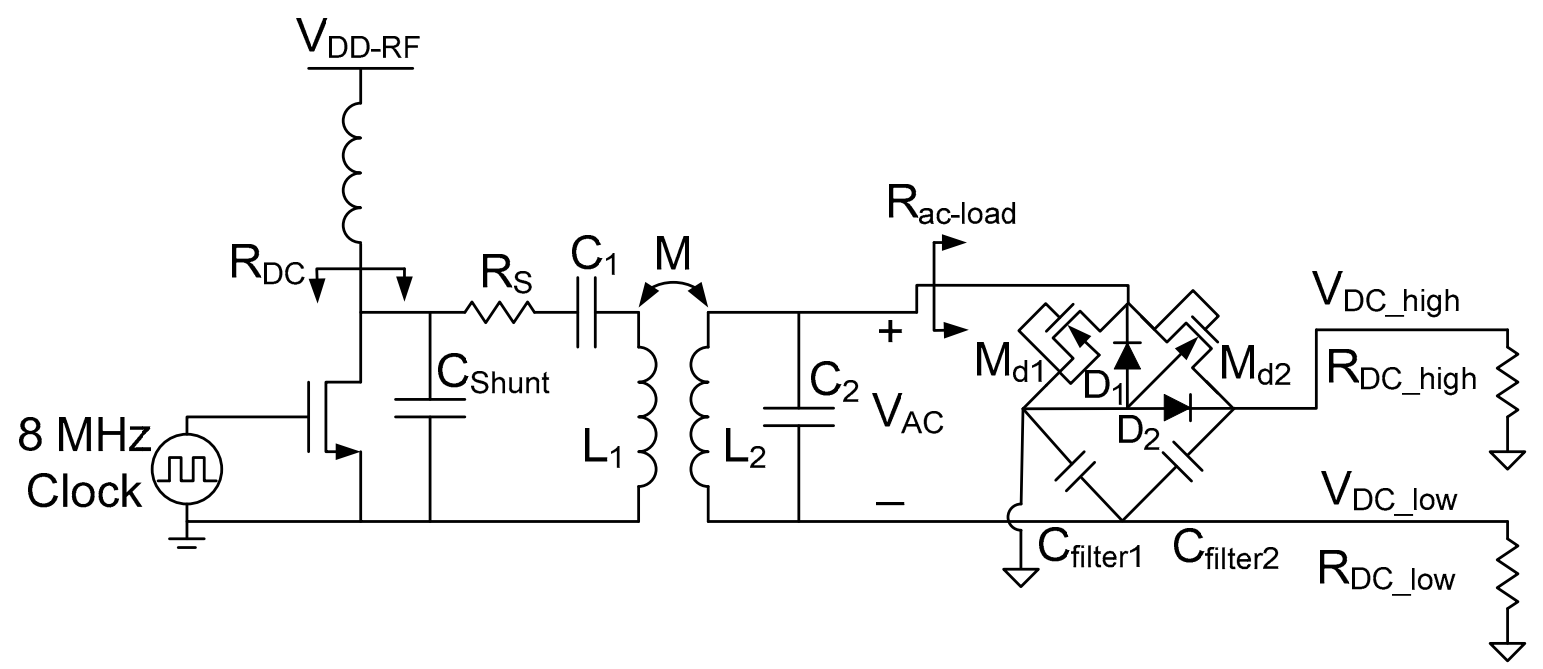

Figure 5-4. RF Powering with Voltage Doubler

The AC resistance, $\mathrm{R}_{\mathrm{ac}-\mathrm{load}}$, of the integrated electronics seen by the tank can be calculated by inspecting the circuit in each of the two half waves of operation: when charging the $V_{D C_{-} \text {low }}$ circuit and when charging the $V_{D C_{-} \text {high }}$ circuit. First, the AC tank voltage is defined as equal to the diode drop plus the DC drop of $\mathrm{V}_{D C}$ low, as shown in Equation 5-7.

$$
V_{A C}=V_{\text {diode_low }}+V_{D C_{-} \text {low }}
$$

The power input to the tank is expressed as the sum of the power dissipated in the diode drop and the power delivered to the $V_{D C_{-} \text {low }}$ circuit, as shown in Equation 5-8.

$$
P_{A C}=P_{\text {diode_low }}+P_{D C_{-} \text {low }}=\frac{V_{A C}^{2}}{2 R_{A C \_l o w}}
$$

The DC power dissipated in the diode can be expressed in term of the current flowing into $V_{D C}$ low, as shown in Equation 5-9.

$$
P_{\text {diode_low }}=V_{\text {diode_low }} \cdot I_{\text {diode }}=V_{\text {diode_low }} \cdot \frac{V_{D C_{\text {_low }}}}{R_{D C_{\text {_llow }}}}
$$

The DC power dissipated in $V_{D C_{-} \text {low }}$ can be expressed in terms of $V_{D C_{-} \text {low }}$ and the total resistive load presented to that supply, as shown in Equation 5-10. 


$$
P_{D C_{-} \text {low }}=\frac{V_{D C \_l o w}^{2}}{R_{D C \_l o w}}
$$

The expressions for $P_{\text {diode_low }}$ and $P_{D C_{-} \text {low }}$ are now are now substituted into Equation 5-8 as shown in Equation 5-11.

$$
\frac{V_{A C}^{2}}{2 R_{A C \_l o w}}=V_{\text {diode_low }} \cdot \frac{V_{D C_{-} \text {low }}}{R_{D C_{\_} \text {low }}}+\frac{V_{D C \_l o w}^{2}}{R_{D C_{\_} \text {low }}}
$$

Equation 5-12 is derived from substituting in Equation 5-7 for $\mathrm{V}_{\mathrm{AC}}$ and factoring out the right side of Equation 5-11,

$$
\frac{\left(V_{\text {diode_low }}+V_{D C \_l o w}\right)^{2}}{2 R_{A C_{-} \text {low }}}=\left(V_{\text {diode_low }}+V_{D C_{-} \text {low }}\right) \cdot \frac{V_{D C \_l o w}}{R_{D C \_l o w}} .
$$

Finally, Equation 5-13 is expressed by solving Equation 5-12 for $R_{A C_{-} l o w}$,

$$
R_{A C_{\_} \text {low }}=\frac{R_{D C \_l o w}}{2} \cdot\left(1+\frac{V_{\text {diode_low }}}{V_{D C \_l o w}}\right) .
$$

The derivation is similar for $V_{D C_{-} \text {high }}$ except the effect of the voltage doubler circuit must be taken into account. First, the tank voltage is defined in the voltage doubler operation as shown in Equation 5-14.

$$
2 V_{A C}=V_{\text {diode_ } h i g h}+V_{D C_{-} \text {high }}
$$

The power input to the tank is expressed as the sum of the power dissipated in the diode drop and the power delivered to the $V_{D C_{-} \text {high }}$ circuit, as shown in Equation 5-15.

$$
P_{A C}=P_{\text {diode_high }}+P_{D C_{-} \text {high }}=\frac{V_{A C}^{2}}{2 R_{A C_{-} \text {high }}}
$$

The DC power dissipated in the diode is expressed in terms of the current flowing into $V_{D C_{-} \text {high }}$, as shown in Equation 5-16. 


$$
P_{\text {diode }}=V_{\text {diode_high }} \cdot I_{\text {diode_high }_{-}}=V_{\text {diode_high }_{-}} \cdot \frac{V_{D C_{-} \text {high }}}{R_{D_{C_{-} \text {high }}}}
$$

The DC power dissipated in $V_{D C_{-} h i g h}$ can be expressed in terms of $V_{D C_{-} \text {high }}$ and the total resistive load presented to that supply, as shown Equation 5-17.

$$
P_{D C_{-} \text {high }}=\frac{V_{D C_{\_} \text {high }}^{2}}{R_{D C_{-} \text {high }}}
$$

The expressions for $P_{\text {diode_high }}$ and $P_{D C_{-} \text {high }}$ are now are now substituted into Equation 5-15 as shown in Equation 5-18.

$$
\frac{V_{A C^{2}}^{2}}{2 R_{A C_{-} h i g h}}=V_{\text {diode } \_ \text {high }} \cdot \frac{V_{D C_{-} \text {high }}}{R_{D C_{-} \text {high }}}+\frac{V_{D C_{-} \text {high }}^{2}}{R_{D_{C_{-} h i g h}}}
$$

Equation 5-19 is expressed by substituting in Equation 5-14 for $\mathrm{V}_{\mathrm{AC}}$ and factoring out the right side of Equation 5-18,

$$
\frac{(1 / 2)^{2}\left(V_{\text {diode_high }}+V_{D C_{-} h i g h}\right)^{2}}{2 R_{A C_{-} h i g h}}=\left(V_{\text {diode_high }}+V_{D C_{-} h i g h}\right) \cdot \frac{V_{D C_{-} h i g h}}{R_{D C_{-} h i g h}} .
$$

Finally, Equation 5-20 is expressed by solving for $R_{A C_{-} \text {high, }}$

$$
R_{A C_{-} \text {high }}=\frac{R_{D C_{-} h i g h}}{8} \cdot\left(1+\frac{V_{\text {diode_high }}}{V_{D C_{-} h i g h}}\right) \text {. }
$$

The total $R_{A C \text {-load }}$ is the parallel combination of these two values calculated in Equations 5-13 and 5-20, as shown in Equation 5-21.

$$
R_{A C-\text { load }}=\frac{R_{D C_{-} \text {low }}}{2} \cdot\left(1+\frac{V_{\text {diode_high }}}{V_{D C_{-} \text {low }}}\right) \| \frac{R_{D C_{\_} \text {high }}}{8} \cdot\left(1+\frac{V_{\text {diode_high }}}{V_{D C_{-} \text {high }}}\right)
$$

\subsection{Analysis of PSK Telemetry System}


Wireless data telemetry is achieved through passive phase-shift keying (PSK) over the same RF link as the inductive powering system. A capacitor, $\mathrm{C}_{\mathrm{SW}}$, is switched in parallel with the secondary RF tank as shown in Figure 3-7. The digitized and Manchester-encoded EMG data bit-stream is used to drive the gate of the switching capacitor. This causes the secondary tank to be de-tuned to a frequency above and below the $8 \mathrm{MHz}$ RF powering frequency. When the secondary circuit is detuned, a complex impedance is reflected back to the primary circuit, causing the voltage across the tank to either lead or lag the phase of the $8 \mathrm{MHz}$ Class-E clock voltage. By mixing the $8 \mathrm{MHz}$ clock with the tank voltage and low-pass filtering the mixer output, the digital phase-shift keying data can be recovered.

The bandwidth of the primary tank, as shown in Figure 5-5 should be about $100 \mathrm{kHz}$ to support the Manchester-encoded 48 kbps data stream, which occupies a spectrum of 48 $\mathrm{kHz}$ to $96 \mathrm{kHz}$. This is data rate is calculated from the redundant signed digit 11 bits of ADC data (22 bits) plus a fixed bit for data alignment and a parity bit for a total of 24 bits sampled at $2 \mathrm{kHz}$. Manchester-encoding doubles the required bandwidth for transmission. A total series resistance $\mathrm{R}_{\mathrm{S}}$ of $2.5 \Omega$, which includes losses in the primary coil $L_{1}$ and $R_{D S}$ of the $R F$ transistor, is required to set the tank $Q$ properly as calculated in Equation 5-22. A resistor, $\mathrm{R}_{\text {Additional, }}$, can be placed in series with $\mathrm{C}_{1}$ and $\mathrm{L}_{1}$ to set the appropriate $\mathrm{R}_{\mathrm{S}}$. 


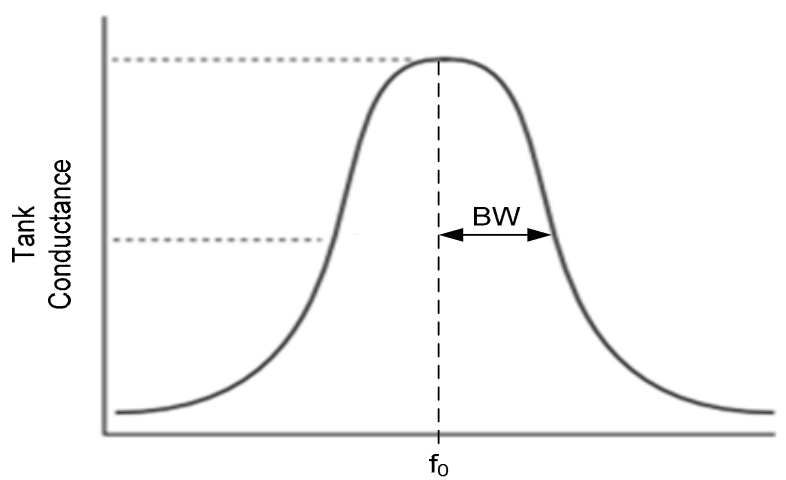

Figure 5-5. Tank Bandwidth

$$
B W=\frac{f_{0}}{2 Q}=\frac{f_{0} R_{S}}{2 \omega L_{1}}=\frac{R_{s}}{2 \cdot 2 \pi L_{1}}=\frac{R_{1}+R_{\text {Additional }}}{2 \cdot 2 \pi L_{1}}
$$

The quality factor of the primary series resonant tank is 40.2 at $8 \mathrm{MHz}$. The quality factor of the unloaded secondary parallel resonant tank using the 30-turn secondary coil is 25.6 at the same frequency. The PSK system was designed to maximize the reflected PSK phase shift, as shown in Figure 5-6. By designing an on-chip switching capacitance $\mathrm{C}_{\mathrm{SW}}$ of $13 \mathrm{pF}$, thereby switching between $50.5 \mathrm{pF}$ and $63.5 \mathrm{pF}$, a $20^{\circ}$ phase shift can be detected at the external PSK demodulator, which mixes together the Class-E clock voltage and the voltage across the tank and series resistance to recover the phase information. 

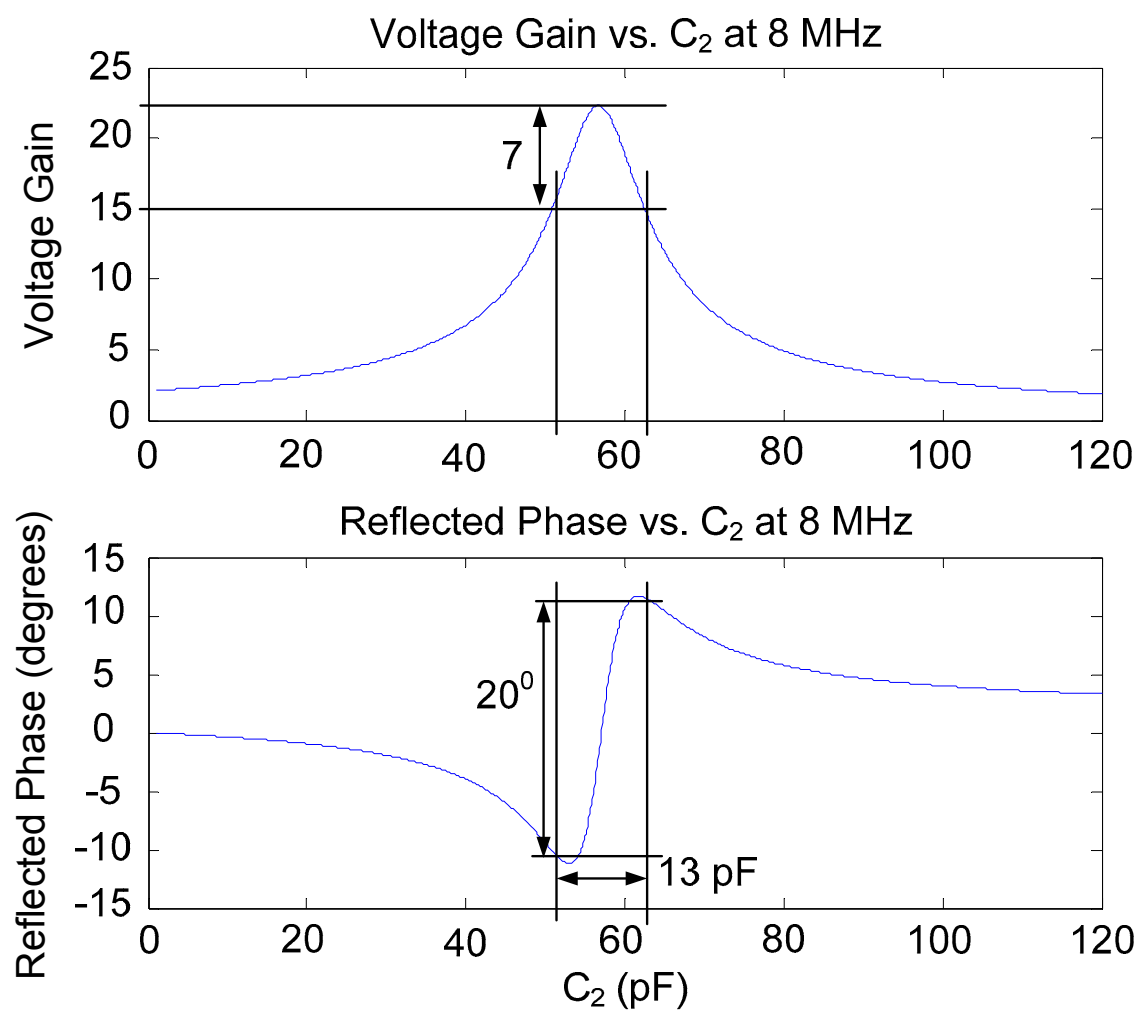

Figure 5-6. PSK Telemetry Maximum Phase Shift

As a consequence of this design, the voltage gain is reduced from about 22 to 15 . Since the wireless power transfer efficiency goes as the square of voltage gain, this causes a significant reduction in wireless power transfer efficiency of about $54 \%$ from the ideal case where the secondary tank is perfectly tuned. A trade-off exists here, such that a lower switching capacitance could be designed to still achieve a detectable phase shift while keeping wireless power transfer efficiency much higher. For example, choosing a $\mathrm{C}_{\mathrm{Sw}}$ of $5 \mathrm{pF}$ for a capacitance range of about $54 \mathrm{pF}$ to $59 \mathrm{pF}$ would provide about 18 degrees of phase shift with only $10 \%$ reduction in voltage gain, corresponding to $17 \%$ reduction in wireless power transfer efficiency. Since the $\mathrm{C}_{\mathrm{SW}}$ is on-chip, this parameter could not be modified after device fabrication. 


\section{$5.660 \mathrm{~Hz}$ Attenuation Investigation}

During the course of testing the EMG amplifier, it was discovered that once EMG electrode leads were attached to the front-end amplifier input pads, a large $60 \mathrm{~Hz}$ coupling caused the front-end amplifier to rail if the entire system was not placed in a shielded box [14]. The $60 \mathrm{~Hz}$ signals are coupled wirelessly from the power lines and various electronics, equipment, and lighting in the lab. Since the signal level caused the amplifier to saturate, the differential nature of the amplifier is unable to compensate for this common-mode signal. In order to combat this effect, termination resistors were added from the front-end amplifier input pads to system ground, as shown in Figure 5-7. If chosen properly, the input termination resistors can still present a high-impedance to the EMG input signal causing a minimal signal loss and at the same time act as a lowimpedance shunt to the $60 \mathrm{~Hz}$ signal to ground, allowing the differential behavior of the EMG amplifier to further attenuate the signal. Adding termination resistors also add thermal noise, so design optimization is required.

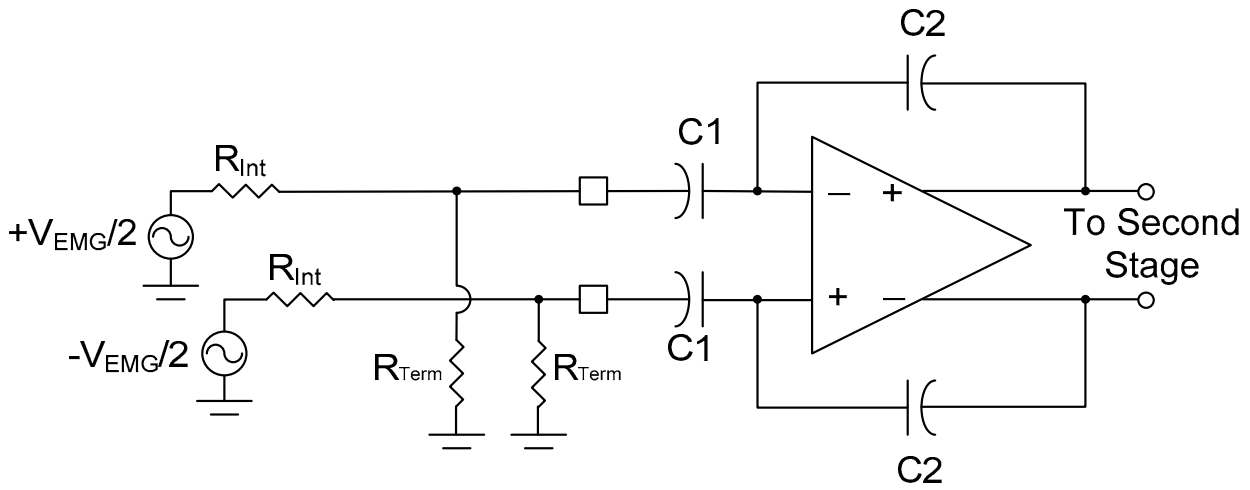

Figure 5-7. $60 \mathrm{~Hz}$ Coupling Model

Adding termination resistors at the OTA input contribute a thermal noise current and change the effective input impedance of the amplifier. A thermal noise voltage is 
developed across the input impedance, which is the termination resistance, $R_{T e r m}$, in parallel with the effective electrode input impedance $R_{\text {Int }}$, as shown in Equation 5-23.

$$
\frac{\overline{v_{i N, R t}^{2}}}{\Delta f}=2 \cdot I_{i N, \text { Rterm }}^{2} \cdot Z_{\text {input }}^{2}
$$

Equation 5-24 is obtained by substituting the current noise and the input impedance in for Equation 5-23.

$$
\frac{\overline{v_{i N, R t}^{2}}}{\Delta f}=2 \cdot \frac{4 k T}{R_{\text {Term }}}\left(\frac{R_{\text {Term }} R_{\text {Int }}}{R_{\text {Term }}+R_{\text {Int }}}\right)^{2}
$$

The voltage input power noise spectral density is obtained in Equation 5-25 by taking the square root of both sides.

$$
\frac{v_{i N, R t}}{\sqrt{\Delta f}}=\sqrt{4 k T \cdot 2 R_{\text {Term }}} \cdot \frac{R_{\text {Int }}}{R_{\text {Term }}+R_{\text {Int }}}
$$

The thermal voltage noise power spectral density contribution of the termination resistors is then added to the electronic thermal noise power spectral density contribution of the interface electronics to obtain the total input-referred noise power spectral density in Equation 5-26.

$$
\frac{v_{i N, \text { Total }}}{\sqrt{\Delta f}}=\sqrt{\frac{\overline{v_{i N, \text { electronic }}^{2}}}{\Delta f}+\frac{\overline{v_{i N, R t}^{2}}}{\Delta f}}
$$

Figure 5-8 shows the input-referred noise floor contribution of adding input termination resistors, assuming the original designed OTA input-referred thermal noise floor of $63 \mathrm{nV} / \sqrt{\mathrm{Hz}}$. From this plot, adding a termination resistance of at least $10 \mathrm{k} \Omega$ causes a negligible increase in the thermal noise floor. 


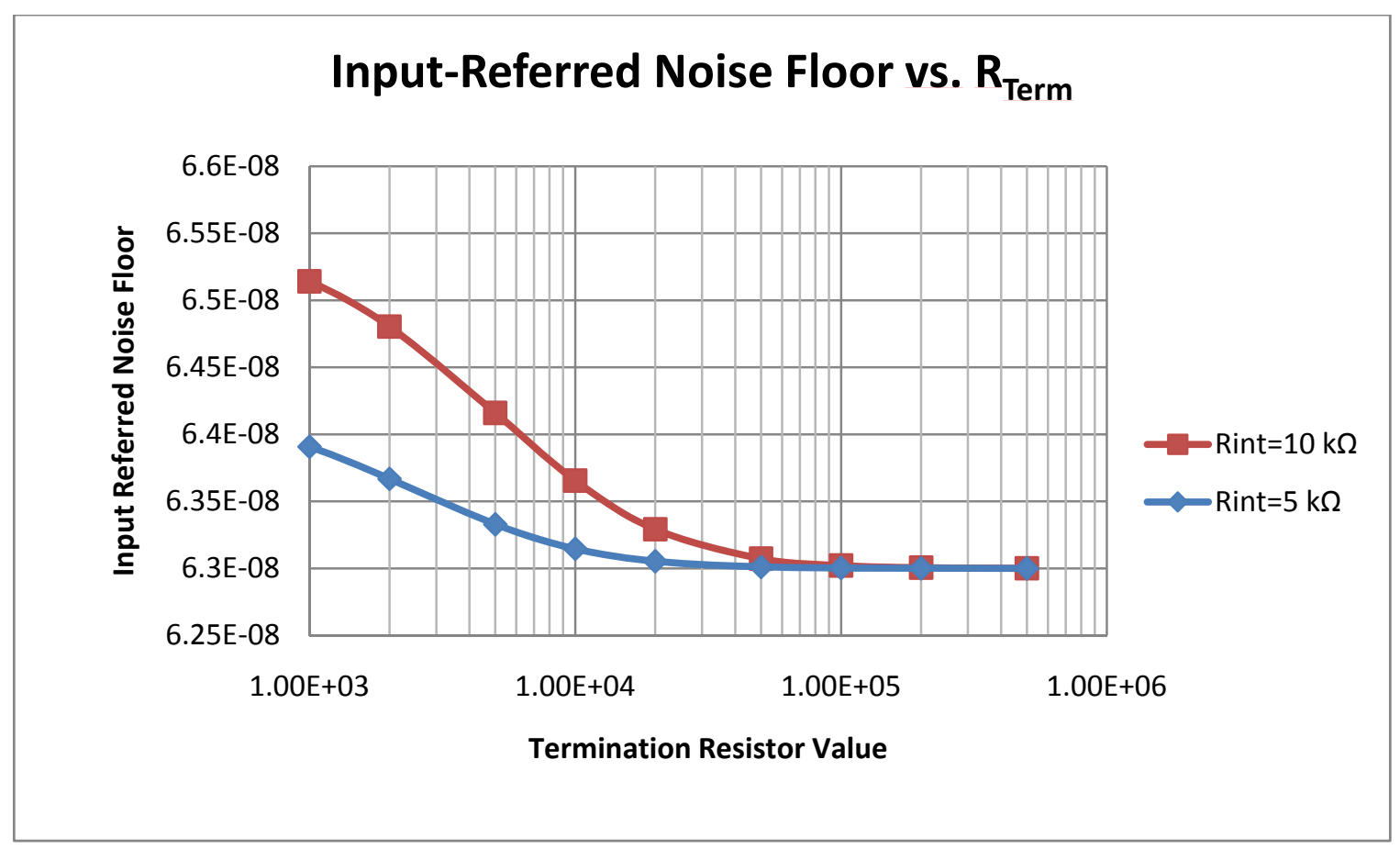

Figure 5-8. Noise Floor vs. Termination Resistor Value

The other effect that must be considered is the effect on the closed-loop signal gain.

Figure 5-9 shows the effect on gain for an interface impedance of either $5 \mathrm{k} \Omega$ or $10 \mathrm{k} \Omega$. By choosing $R_{\text {Term }} / R_{\text {Int }}=3,75 \%$ of the signal gain is retained. By choosing $R_{\text {Term }} / R_{\text {Int }}=$ 9 , over $90 \%$ of the signal gain is retained. 


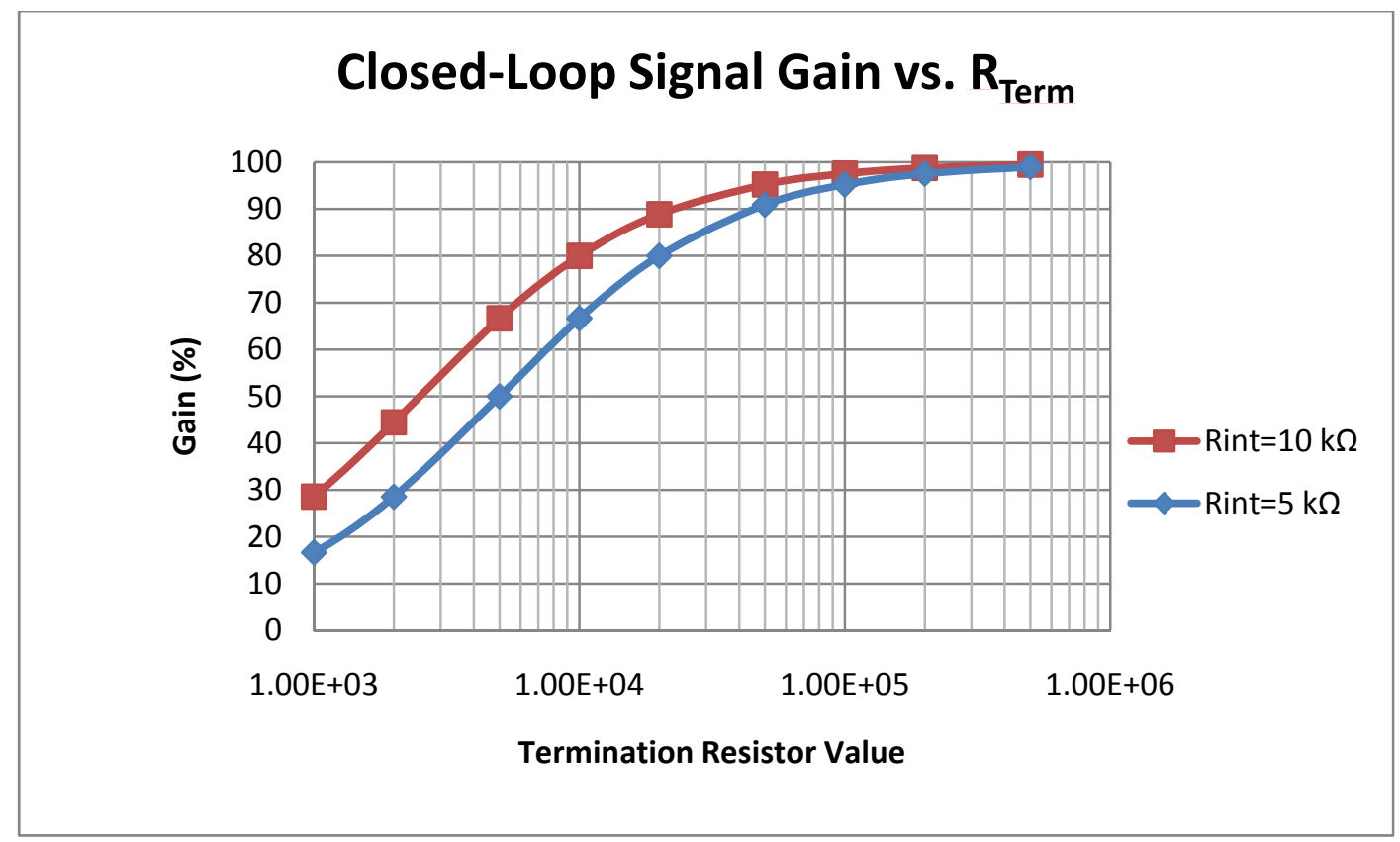

Figure 5-9. Closed-Loop Signal Gain vs. Termination Resistor Value

A termination resistance of $R_{\text {Term }}=50 \mathrm{k} \Omega$ is chosen as a reasonable value to retain most of the signal level while greatly attenuating $60 \mathrm{~Hz}$ interference. This resistor selection allows for long EMG electrode leads over 1 meter in length to be used while still recording a high resolution EMG waveform.

\subsection{Summary}

A complete wireless near field inductive powering and passive data telemetry system design has been presented in this chapter. A Class-E amplifier is used to drive the external tank and was selected for its high efficiency operation. A number of RF inductors coils were built to satisfy the geometry requirements of this system. These coils were characterized and their coupling factors were measured in order to predict wireless power transfer efficiency. The coil which enabled maximum efficiency was selected for use in this system. An equation to determine the AC load that the integrated 
electronics presents to the secondary tank was also presented. Finally, the trade-off in selection of input termination resistors to counteract the effects of $60 \mathrm{~Hz}$ interference was investigated and a termination resistance of $50 \mathrm{k} \Omega$ is chosen to minimize $60 \mathrm{~Hz}$ coupling while contributing a minimal reduction of closed-loop signal gain and negligible increase in thermal noise floor. 
Chapter 6

Measurement Results

\subsection{Overview}

The EMG sensing electronics were designed as an integrated circuit in the AMI 1.5 $\mu \mathrm{m}$ two-metal, two-poly CMOS process and fabricated through the MOSIS Service. This chapter presents the characterization and measurement results for this chip and for the overall wireless EMG sensing microsystem.

\subsection{System Characterization}

A micrograph of the fabricated ASIC is presented in Figure 6-1. The building blocks, including the low-noise amplifier, $2.5 \mathrm{x}$ gain stage, $\mathrm{ADC}$, RF to $\mathrm{DC}$ rectification and power regulation circuitry with passive digital PSK data telemetry and digital state machine and ADC control blocks, are labeled. 


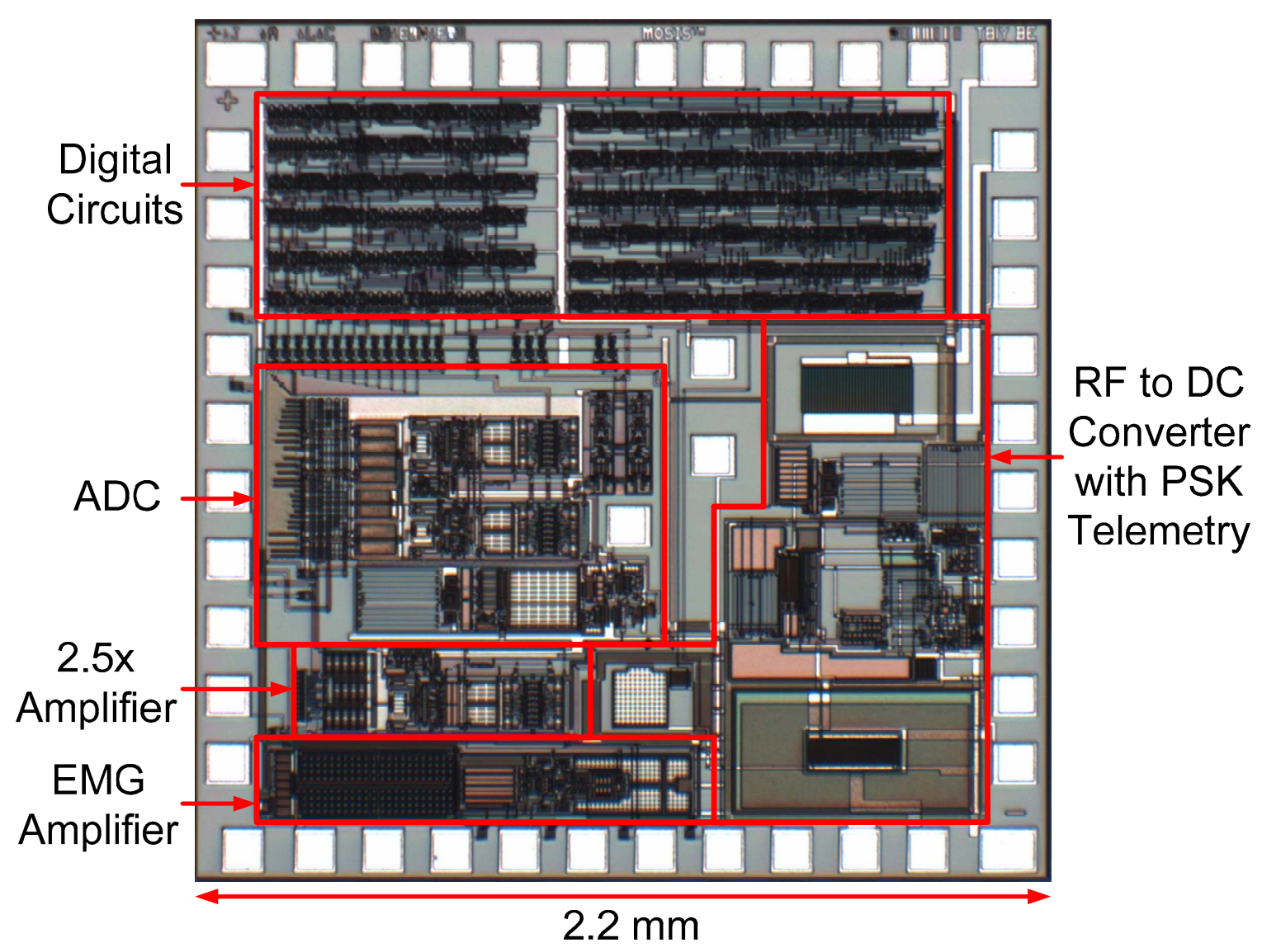

Figure 6-1. ASIC Microphotograph

Characterization begins with the integrated electronics by examining the front-end EMG amplifier. The differential output of the OTA is buffered through an on-chip PMOS follower biased with $50 \mathrm{k} \Omega$ off-chip load resistors. These output buffers have a voltage gain of 0.86 . The buffered differential output is then converted to a single-ended signal with a precision unity-gain AD620 instrumentation amplifier.

An HP Dynamic Signal Analyzer was used to perform a swept sinusoidal response curve and measure the frequency response of the OTA as shown in Figure 6-2. The OTA -3dB low-pass corner frequency was designed to be $1 \mathrm{kHz}$, and was measured to be $920 \mathrm{~Hz}$. This is due to a reduced OTA reference current, which is mirrored from a band-gap reference in the RF/DC power conversion block. The n-well resistors in the 
band-gap reference were fabricated with a dimension smaller than drawn in the layout, causing an increased resistance and reduced current. The high-pass corner frequency was measured to be $20 \mathrm{mHz}$, which is within the expected range.

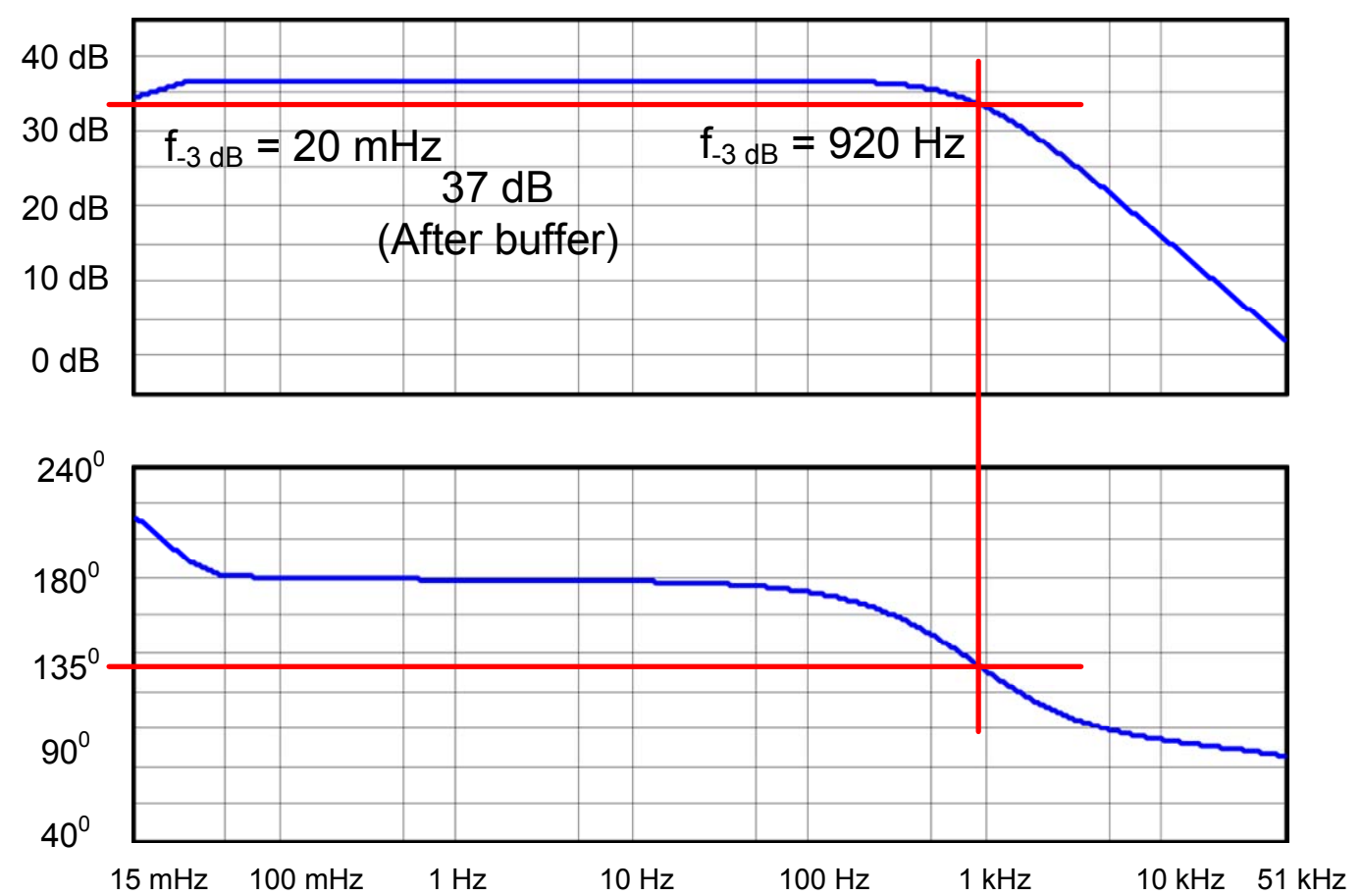

Figure 6-2. EMG Amplifier Frequency Response

The OTA output thermal noise floor was measured by shorting the differential inputs of the OTA to ground and measuring the output. The designed input-referred noise floor is $63 \mathrm{nV} / \sqrt{\mathrm{Hz}}$, which is equivalent to a $5 \mu \mathrm{V} / \sqrt{\mathrm{Hz}}$ output-referred noise floor. The measured noise floor at the output of the buffer is $5.5 \mu \mathrm{V} / \sqrt{H z}$, which is equivalent to $6.4 \mu \mathrm{V} / \sqrt{\mathrm{Hz}}$ at the OTA output and $78 \mathrm{nV} / \sqrt{\mathrm{Hz}}$ referred to the OTA input. This is again due to the reduced OTA bias current. The measured thermal noise floor is shown in Figure 6-3. 


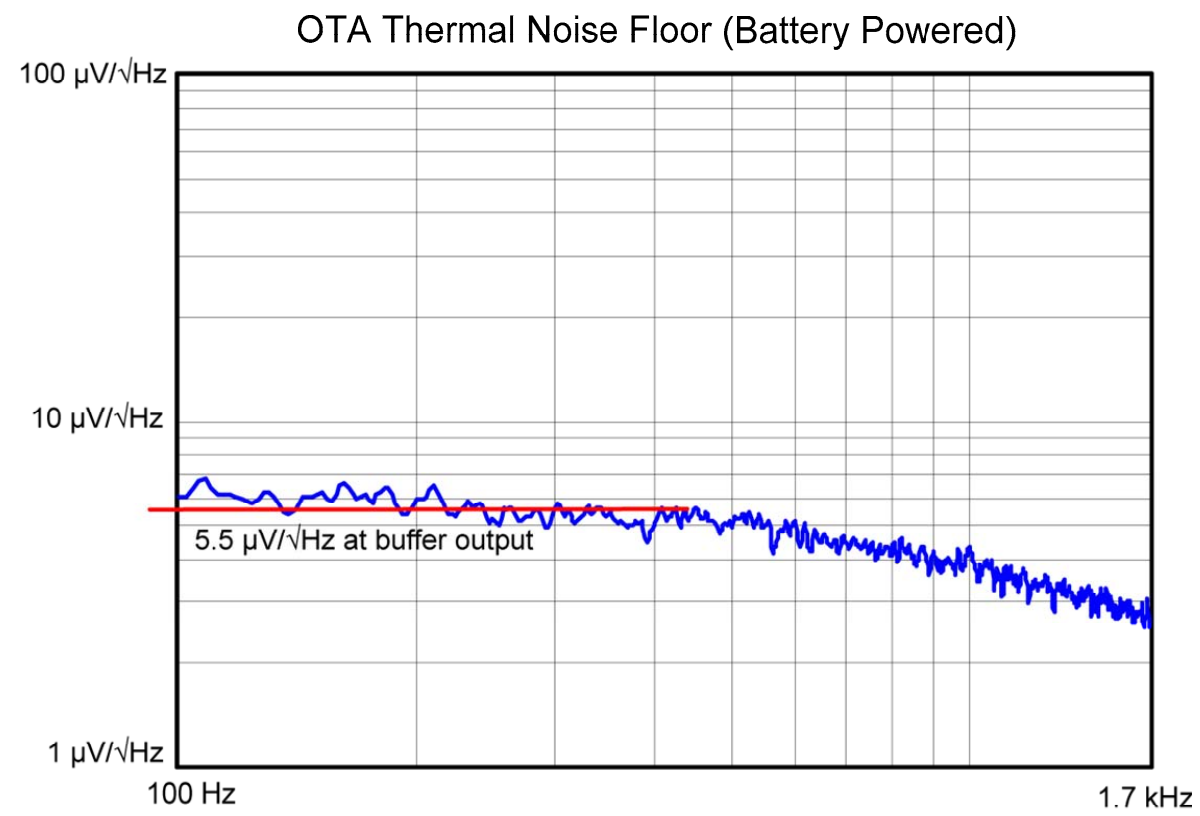

Figure 6-3. EMG Amplifier Output Thermal Noise Floor

Figure 6-4 shows the OTA $1 / f$ noise corner frequency, where the flicker noise is equal to the thermal noise, is equal to $30 \mathrm{~Hz}$. The OTA was designed for a $1 / f$ noise corner frequency of $20 \mathrm{~Hz}$. The thermal noise is slightly higher than the designed value due to the reduced OTA bias current.

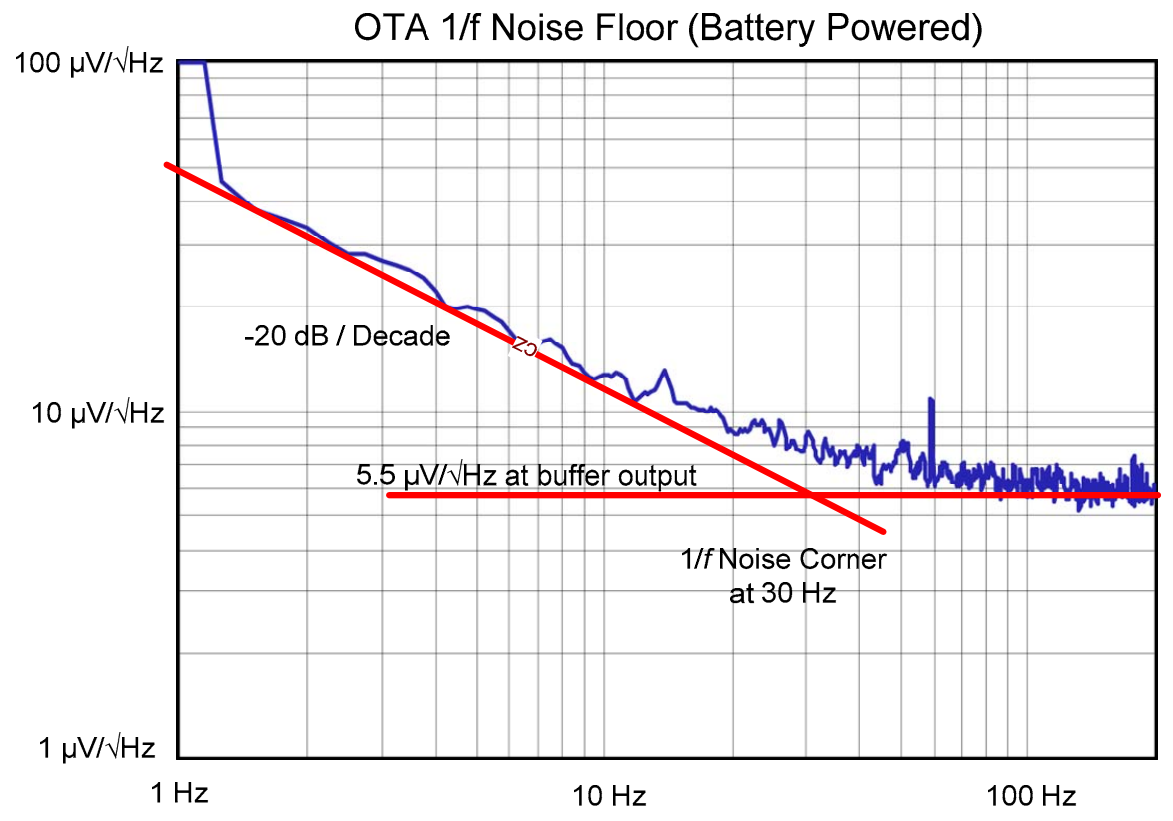

Figure 6-4. EMG Amplifier 1/f Noise Corner Frequency 
In order to characterize the EMG signal chain together with the ADC, a single tone was applied to the OTA input and the ADC digital output was recorded. An example of ADC output spectrum with the OTA driven by a $137 \mathrm{~Hz}$ tone with peak-topeak amplitude of $500 \mu \mathrm{V}_{\mathrm{P}-\mathrm{P}}$ is shown in Figure 6-5.

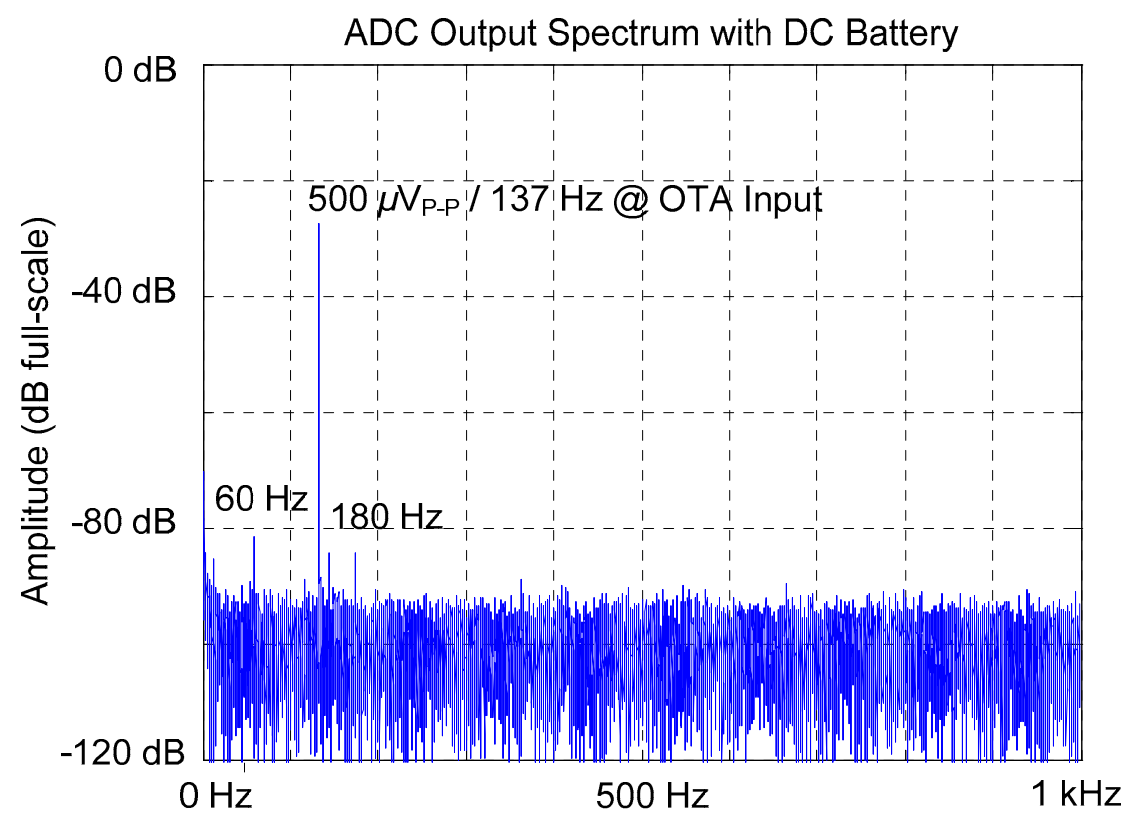

Figure 6-5. ADC Output Spectrum with DC Battery

From the ADC output data, the signal to noise ratio (SNR) can be measured and used to calculate the effective number of bits (ENOB) of the system by Equation 6-1.

$$
E N O B=\frac{(S N R-1.76)}{6.02}
$$

For this system, the OTA front-end amplifier and 2.5x gain stage were designed for an 11-bit dynamic range (DR). The ratio of the EMG signal chain's full-scale differential output voltage swing at the output of the $2.5 \mathrm{x}$ gain stage to the total voltage noise, generated by the OTA and $2.5 \mathrm{x}$ gain stage, and referred to the output of the $2.5 \mathrm{x}$ gain stage, was designed to be better than 11 bits. This 11-bit SNR design calculation is 
presented in Equation 6-2, with the "DC" SNR defined as the $2 \mathrm{~V}$ full-scale differential peak-to-peak ADC input voltage swing to the RMS voltage noise ratio.

$$
S N R_{A D C, D C}=\frac{\mathrm{V}_{P P, F S}}{v_{i N, A D C, D C}}>D R=2^{11} \rightarrow v_{i N, A D C, D C}<\frac{2 \mathrm{~V}}{2048}=1 \mathrm{mV}
$$

Both the OTA and 2.5x gain stage were designed such that the total integrated output noise referred to the output of the $2.5 \mathrm{x}$ gain stage (the input of the ADC) was less than $1 \mathrm{mV}$. However, ENOB is calculated with the signal power instead of the signal amplitude. This design discrepancy causes lower than originally expected ENOB performance. Using the correct expression, the noise requirement should be $0.35 \mathrm{mV}_{\mathrm{RMS}}$ at the $\mathrm{ADC}$ input.

$$
\begin{gathered}
v_{i N, A D C, 11-b i t}<\frac{V_{P} / \sqrt{2}}{2^{11}}=\frac{0.707 \mathrm{mV}_{R M S}}{2048}=0.35 \mathrm{mV}_{R M S} \\
S N R_{11-b i t}=6.02 \times 11 \text { bit }+1.76=68 \mathrm{~dB}
\end{gathered}
$$

The measured $\mathrm{ADC}$ stand-alone SNR performance was $64 \mathrm{~dB}$, corresponding to an ENOB performance of 10.35 bits. The equivalent ADC input-referred noise can be calculated from the measured ADC SNR, as shown in Equation 6-5.

$$
v_{i N, A D C}=\frac{V_{P} / \sqrt{2}}{S N R}=\frac{1 / \sqrt{2}}{64 \mathrm{~dB}}=\frac{.707 \mathrm{mV}_{R M S}}{1585}=0.45 \mathrm{mV}_{R M S}
$$

The $2.5 \mathrm{x}$ gain stage is designed to have an integrated output noise, $v_{o N, 2.5 x}$, of 0.4 $\mathrm{mV}_{\text {RMS. }}$. By summing the noise power of the $2.5 \mathrm{x}$ gain stage and the $\mathrm{ADC}$, the combined SNR performance of the $2.5 \mathrm{x}$ gain stage and the ADC can be calculated, as shown in Equation 6-6. 


$$
\begin{aligned}
& S N R_{2.5 x+A D C}=\frac{V_{p} / \sqrt{2}}{\sqrt{v_{i N, A D C}^{2}+v_{O N, 2.5 x}^{2}}} \\
& S N R_{2.5 x+A D C}=\frac{.707 \mathrm{mV}_{R M S}}{\sqrt{0.45 \mathrm{mV}_{R M S}^{2}+0.4 \mathrm{mV}_{R M S}^{2}}}=1170=61.4 \mathrm{~dB}
\end{aligned}
$$

From the $2.5 \mathrm{x}$ gain stage and ADC combined SNR performance, the corresponding ENOB performance can also be calculated, as in Equation 6-7.

$$
E N O B_{2.5 x+A D C}=\frac{(61.4-1.76)}{6.02}=9.9 \text { bits }
$$

The SNR performance when the entire system is driven at the OTA differential input can also be predicted. The OTA output integrated noise, $v_{\text {ON,OTA, }}$, is equal to 0.25 $\mathrm{mV}_{\mathrm{RMS}}$, which is equivalent to $v_{o N, 2.5 x}=v_{O N, O T A} \times 2.5=0.6 \mathrm{mV}_{\mathrm{RMS}}$ at the $2.5 \mathrm{x}$ gain stage output, at the ADC input.

$$
\begin{aligned}
& S N R_{\text {System }}=\frac{V_{p} / \sqrt{2}}{\sqrt{v_{i N, A D C}^{2}+v_{\text {ON, }, 2.5 x}^{2}+\left(2.5 \cdot v_{\text {ON }, \text { OTA }}\right)^{2}}} \\
& S N R_{\text {System }}=\frac{.707 \mathrm{mV}_{R M S}}{\sqrt{0.45 \mathrm{mV}_{R M S}^{2}+0.4 \mathrm{mV}_{R M S}^{2}+0.6 \mathrm{mV}_{R M S}^{2}}}=832=58.4 \mathrm{~dB}
\end{aligned}
$$

From the entire system combined SNR performance, the corresponding ENOB performance can also be calculated, as in Equation 6-9.

$$
E N O B_{\text {System }}=\frac{(58.4-1.76)}{6.02}=9.4 \text { bits }
$$

This expected ENOB performance matches closely to the measured performance, which is presented in Figure 6-6. The measured ADC performance is 10.35 ENOB. The measured performance when the system is driven at the input of the $2.5 \mathrm{x}$ gain is 9.75 ENOB, compared to the expected level of 9.9 ENOB based on the $2.5 \mathrm{x}$ gain stage's calculated electronic noise floor. When the entire system is tested by driving a 
differential signal at the OTA input at various frequencies spaced over the system bandwidth, the repeatable measured performance is $9.35 \mathrm{ENOB}$, compared to the expected level of 9.4 ENOB considering the OTA's thermal noise contribution.

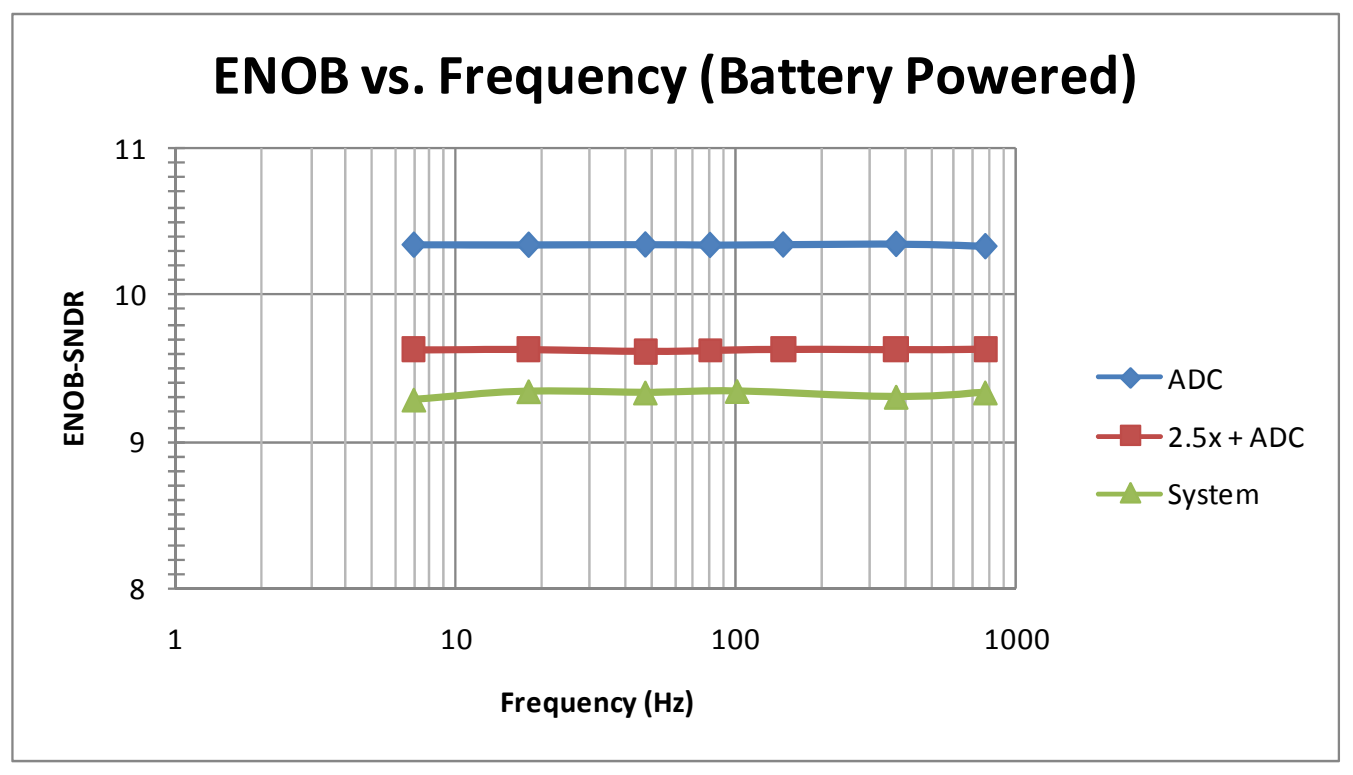

Figure 6-6. Measured Effective Number of Bits

If the entire OTA $+2.5 \mathrm{x}$ gain stage $+\mathrm{ADC}$ system is re-tested using power provided from the RF/DC power conversion block under either $\mathrm{AC}$ powering (an $\mathrm{RF}$ signal is applied directly to the secondary tank) or wireless RF powering (by transferring power wirelessly through inductive coupling), the performance is slightly degraded, as shown in Figure 6-7. With AC powering, the system performance is about 8.9 ENOB. Under wireless RF powering, the system performance is about 8.6 ENOB. 


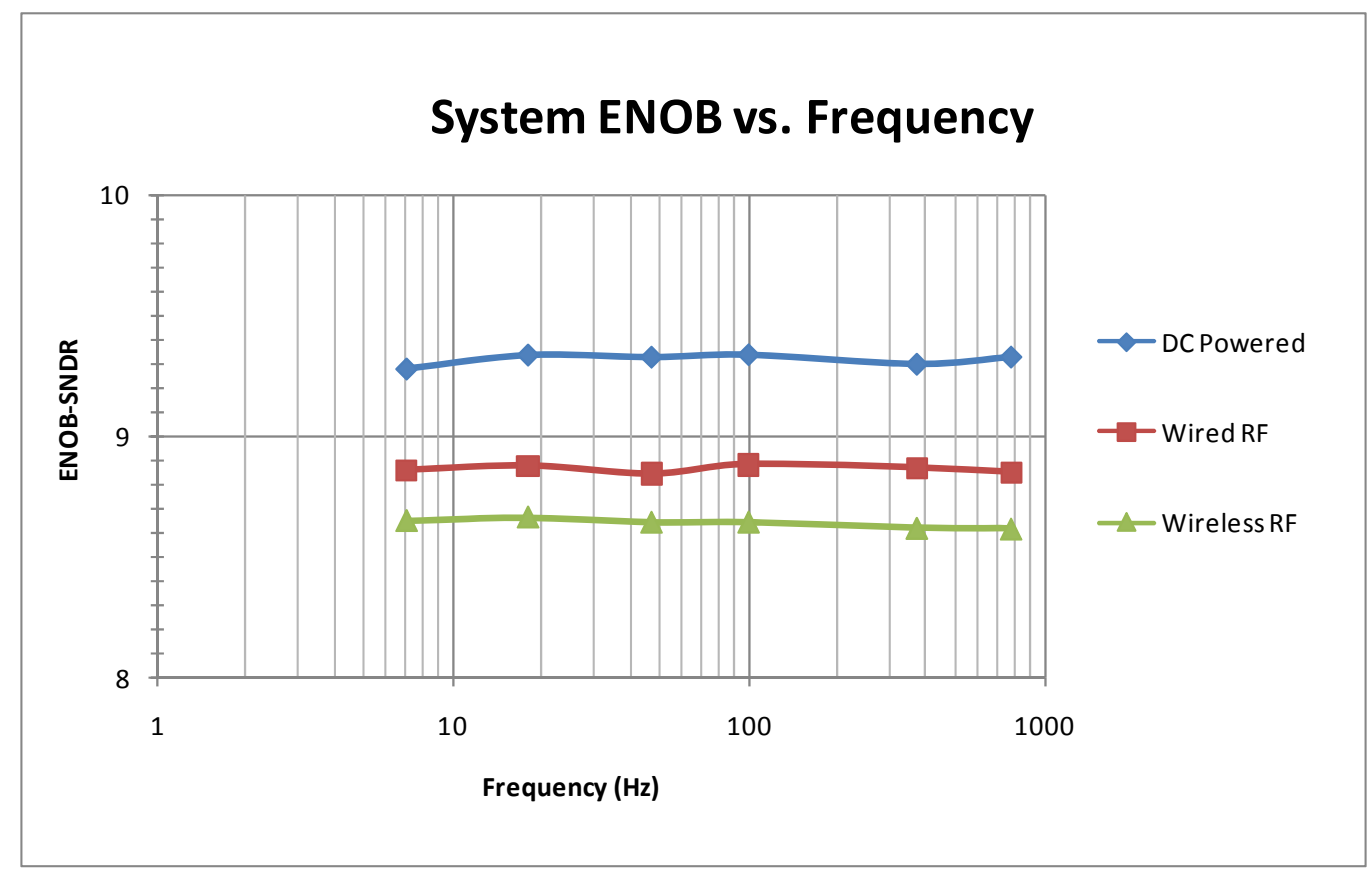

Figure 6-7. System ENOB Effect due to Powering Schemes

When the system is under RF powering, the noise floor at the ADC digital output is increased, causing a reduced SNR and a degraded ENOB performance. There are also additional spurious tones in the output spectrum in this setup, as shown in Figure 6-8.

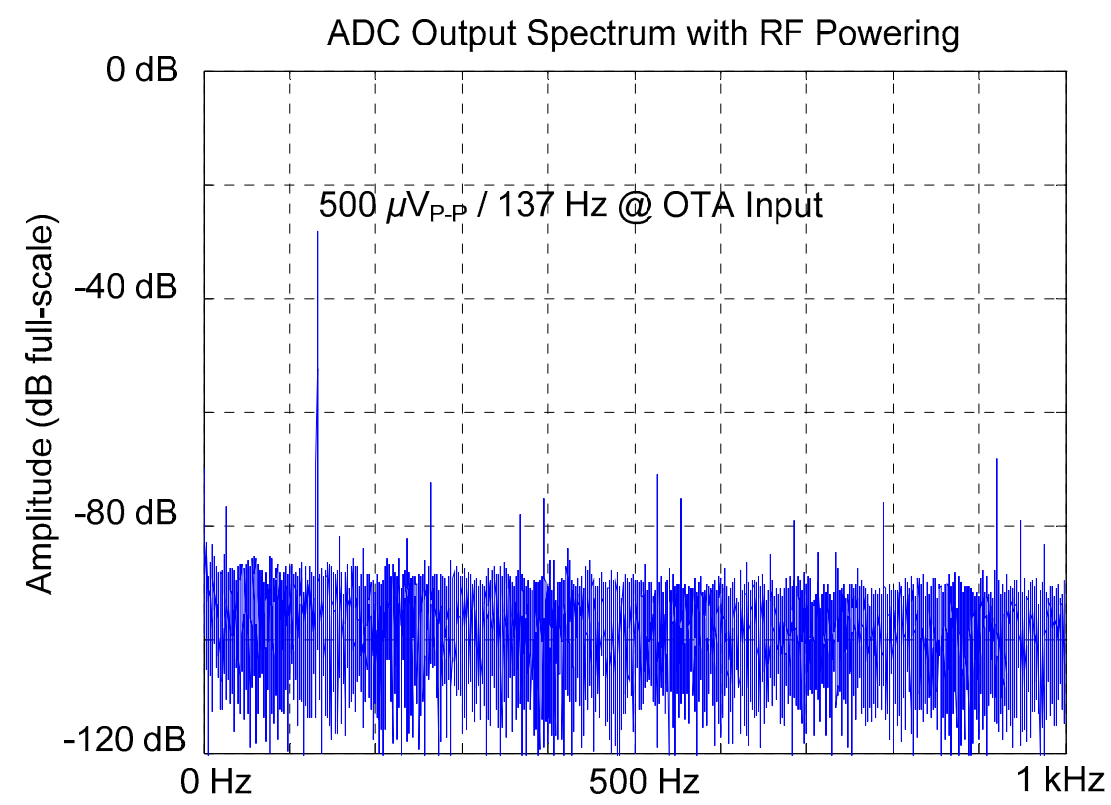

Figure 6-8. ADC Output Spectrum with RF Powering 
The implantable IC consumes $63.2 \mu \mathrm{A}$ from the $V_{\text {supply_low }}$ of $2.5 \mathrm{~V}$ and $19.8 \mu \mathrm{A}$ from $V_{\text {supply_high }}$ of $4.5 \mathrm{~V}$. These supplies are derived from the voltage doubler, as shown in as detailed in Figure 5-4. The doubler requires a $6.5 \mathrm{~V}_{\text {P-P }}\left(3.25 \mathrm{~V}_{\text {Peak }}\right) \mathrm{AC}$ waveform across the LC tank input to power up the system, as tested experimentally by using a function generator directly attached to the secondary tank input. The $V_{\text {diode_low }}$ drop from $\mathrm{M}_{\mathrm{d} 1}$ is $0.75 \mathrm{~V}$ and the $V_{\text {diode }}$ high drop from the combination of $\mathrm{M}_{\mathrm{d} 1}$ and $\mathrm{M}_{\mathrm{d} 2}$ is $1.25 \mathrm{~V}$. From these supply voltages and currents, the loads may be modeled as resistances with $\mathrm{R}_{\mathrm{DC} \_ \text {Low }}$ of $39.6 \mathrm{k} \Omega$ and $\mathrm{R}_{\mathrm{DC} \_ \text {High }}$ of $227 \mathrm{k} \Omega$. Based on these $\mathrm{DC}$ loads and diode drops, equivalent AC loads may be computed as shown in Equations 6-10 and 6-11.

$$
\begin{aligned}
& R_{A C_{\_} \text {low }}=\frac{R_{D C_{\_} \text {low }}}{2} \cdot\left(1+\frac{V_{\text {diode_low }}}{V_{D C_{\_} \text {low }}}\right)=25.7 \mathrm{k} \Omega \\
& R_{A C_{\_} \text {high }}=\frac{R_{D C_{\_} \text {high }}}{8} \cdot\left(1+\frac{V_{\text {diode_high }}}{V_{D C_{\_} \text {high }}}\right)=36.3 \mathrm{k} \Omega
\end{aligned}
$$

Based on these $\mathrm{AC}$ loads, an equivalent system $\mathrm{AC}$ load presented to the secondary tank can be calculated as Equation 6-12.

$$
R_{A C \text {-lood }}=\frac{R_{D C \text { _low }}}{2} \cdot\left(1+\frac{V_{\text {diode_low }}}{V_{D C_{-} \text {low }}}\right) \| \frac{R_{D C \text { _high }}}{8} \cdot\left(1+\frac{V_{\text {diode_high }}}{V_{D C_{-} \text {high }}}\right)=15 \mathrm{k} \Omega
$$

The data presented in Table 6-1 shows the calculated, simulated, and measured parameters of the Class-E amplifier used in this design. The external primary coil $\mathrm{L}_{1}$ is a 2 diameter, 10-turn spiral coil and the design follows the equations in [12]. The RF switching transistor is a Freescale MRF6V2010NR1, which is an n-channel enhancement mode lateral MOSFET designed as large-signal output driver for frequencies up to 450 MHz. The condition for the design is to deliver $350 \mu \mathrm{W}$ into $\mathrm{R}_{\mathrm{AC} \_ \text {Load }}$ from the secondary tank at $1 \mathrm{~cm}$ separation distance with good alignment at $8 \mathrm{MHz}$ operating frequency. 
Simulated $\mathrm{R}_{\mathrm{DC}}$ is calculated from the average current into the choke inductor from an ideal power supply. Measured $\mathrm{R}_{\mathrm{DC}}$ is obtained by observing the current supplied by a bench-top power supply when powering the tuned Class-E amplifier circuit. The Class-E circuit does not have ideal zero-voltage-switching behavior as shown in the scope plot, so the Class-E amplifier requires additional power (higher $\mathrm{R}_{\mathrm{DC}}$ and therefore higher $\mathrm{V}_{\mathrm{DD}}$ ) to deliver the same power to the AC load.

Table 6-1. Class-E Amplifier Parameters

\begin{tabular}{|c|c|c|c|}
\hline Parameter & Calculated & Simulated & Measured \\
\hline $\mathrm{L}_{1}$ & $2 \mu \mathrm{H}$ & $2 \mu \mathrm{H}$ & $2 \mu \mathrm{H}$ \\
\hline $\mathrm{C}_{1}$ & $204 \mathrm{pF}$ & $204 \mathrm{pF}$ & $204 \mathrm{pF}$ \\
\hline $\mathrm{C}_{\text {Shunt }}$ & $1460 \mathrm{pF}$ & $1460 \mathrm{pF}$ & $1460 \mathrm{pF}$ \\
\hline $\mathrm{R}_{\mathrm{S}}$ & $2.5 \Omega$ & $2.5 \Omega$ & $2.5 \Omega$ \\
\hline $\mathrm{R}_{\mathrm{DC}}$ & $4.4 \Omega$ & $5.8 \Omega$ & $7 \Omega$ \\
\hline $\mathrm{V}_{\text {Drain,P }} / \mathrm{V}_{\mathrm{DD}}$ & 3.56 & 3.43 & 3.4 \\
\hline $\mathrm{V}_{\mathrm{DD}, 1-\mathrm{cm}}$ & $138 \mathrm{mV}$ & $160 \mathrm{mV}$ & $175 \mathrm{mV}$ \\
\hline
\end{tabular}


The plot shown in Figure 6-9 shows the simulated RF FET drain voltage waveform in the Class-E amplifier. Figure 6-10 shows a measured Class-E amplifier drain waveform.

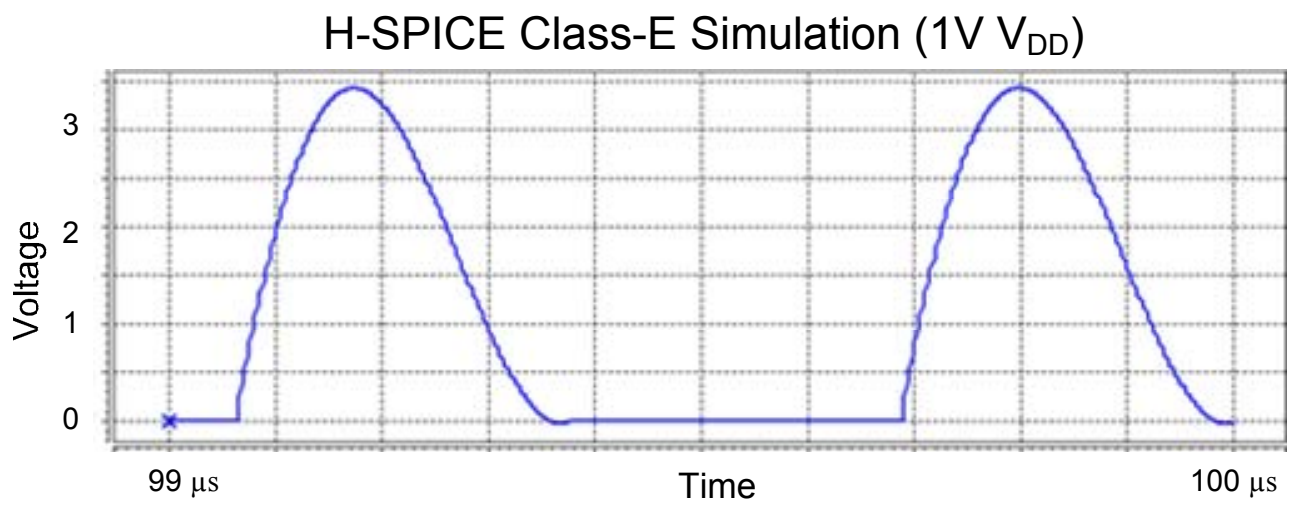

Figure 6-9. Simulated Class-E Waveform

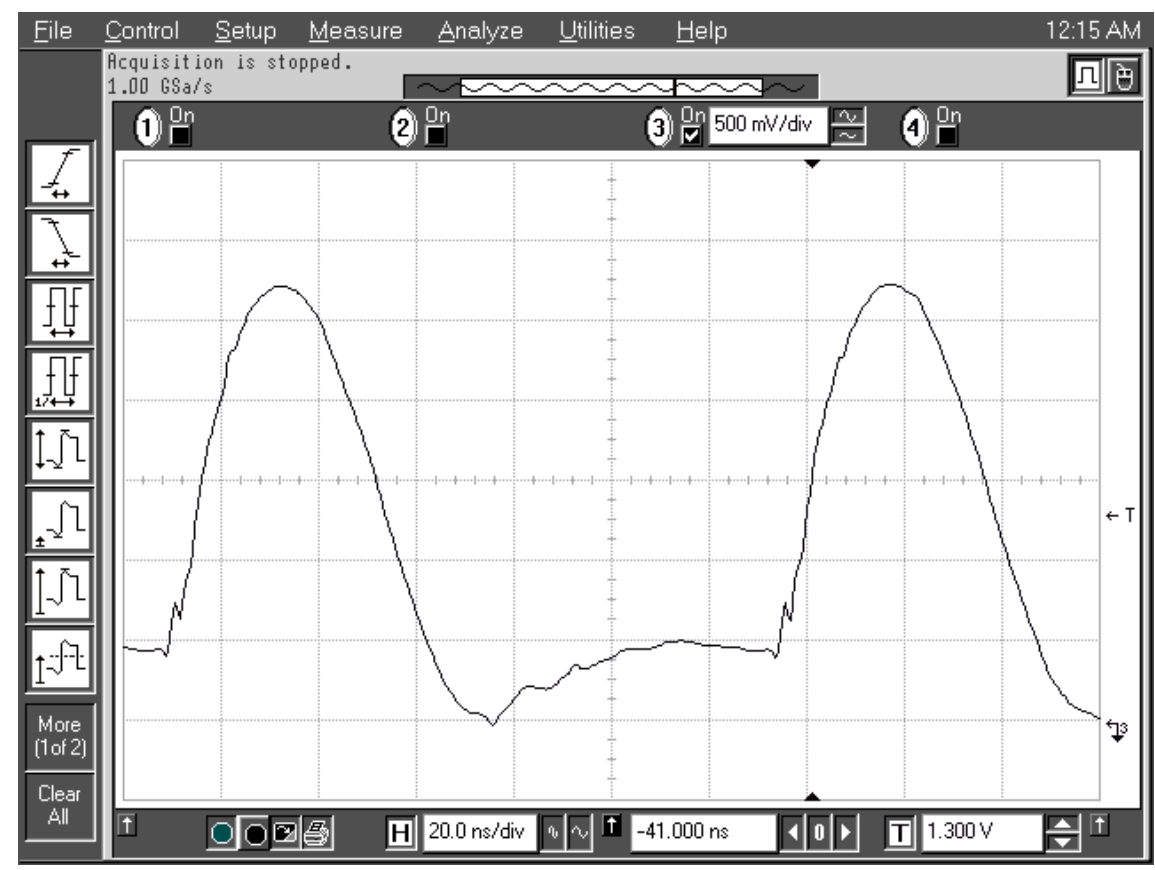

Figure 6-10. Class-E Drain Waveform

The Class-E DC supply voltage requirement may be low to support wireless operation of the implantable IC, in the range of 100 to $200 \mathrm{mV}$ when the coils are at $1 \mathrm{~cm}$ separation distance and well-aligned. A test was performed to verify that the Class-E 
amplifier could continue to operate at low supply voltage levels. If the Class-E amplifier is operating properly, the ratio of the peak of the drain to source voltage to the DC supply voltage should remain a constant of around 3.4[7][9]. The plot in Figure 6-11 shows the results of this test with a linear relationship and small intercept offset, showing that the Class-E amplifier can operate with a low DC supply voltage.

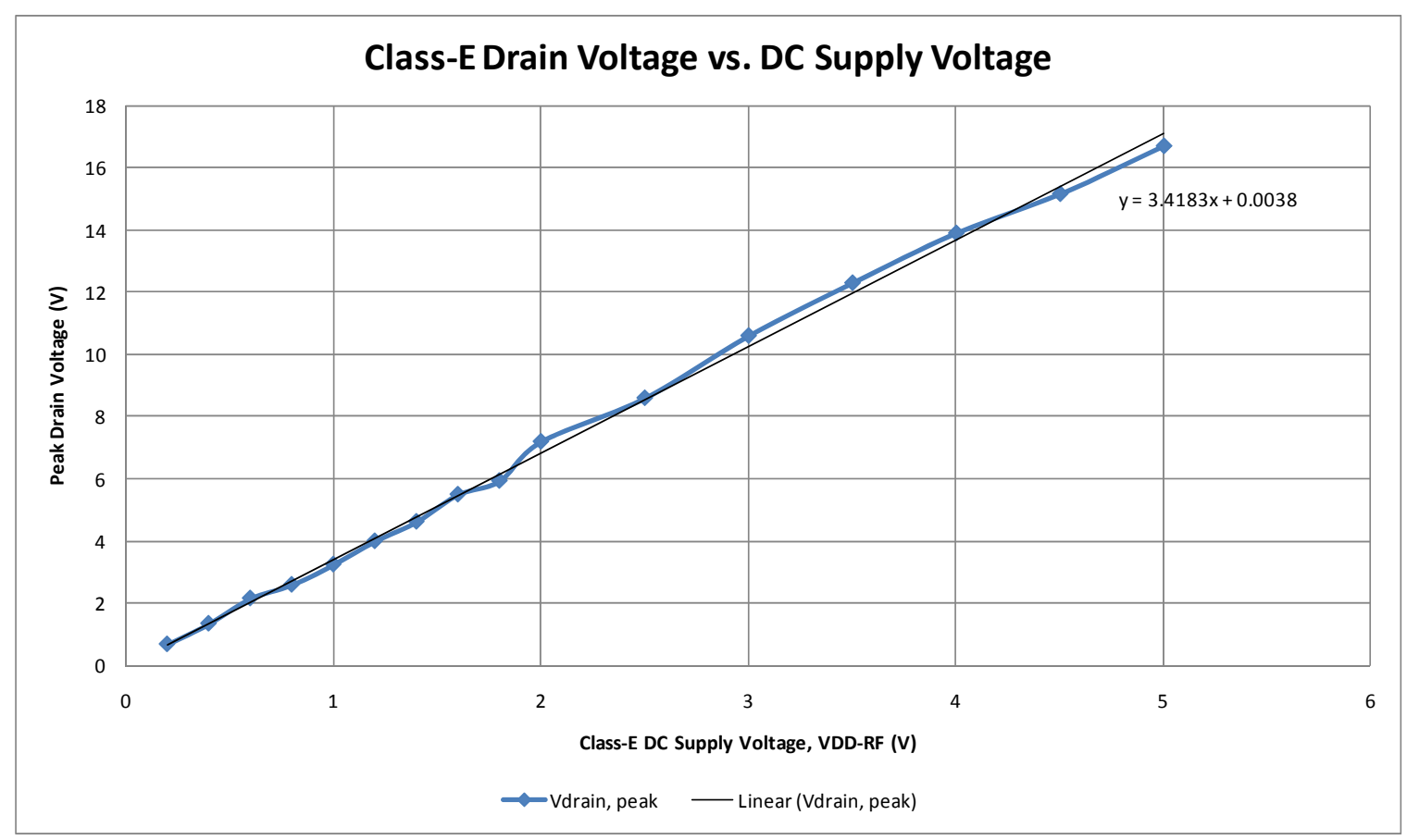

Figure 6-11. Class-E Drain Voltage vs. DC Supply Voltage

The predicted wireless power transfer efficiency data shown in Figure 5-3 assumed an $\mathrm{R}_{\mathrm{AC}-\mathrm{Load}}$ of $1.95 \mathrm{k} \Omega$. The wireless power transfer efficiency can be reestimated with the updated load resistance of $15 \mathrm{k} \Omega$ and is shown in Figure 6-12. The new power transfer curve shows that the expected efficiency at $8 \mathrm{MHz}$ is reduced from $10 \%$ to $8.65 \%$ without reducing the primary tank Q for PSK data telemetry bandwidth requirement, and down to $8.25 \%$ when the $\mathrm{Q}$ is reduced. It also shows that an increased power transfer efficiency is possible if an operating frequency of $16 \mathrm{MHz}$ is selected. 
The two $15 \mathrm{k} \Omega$ curves show the difference in performance when the primary tank $\mathrm{Q}$ is reduced for PSK operation.

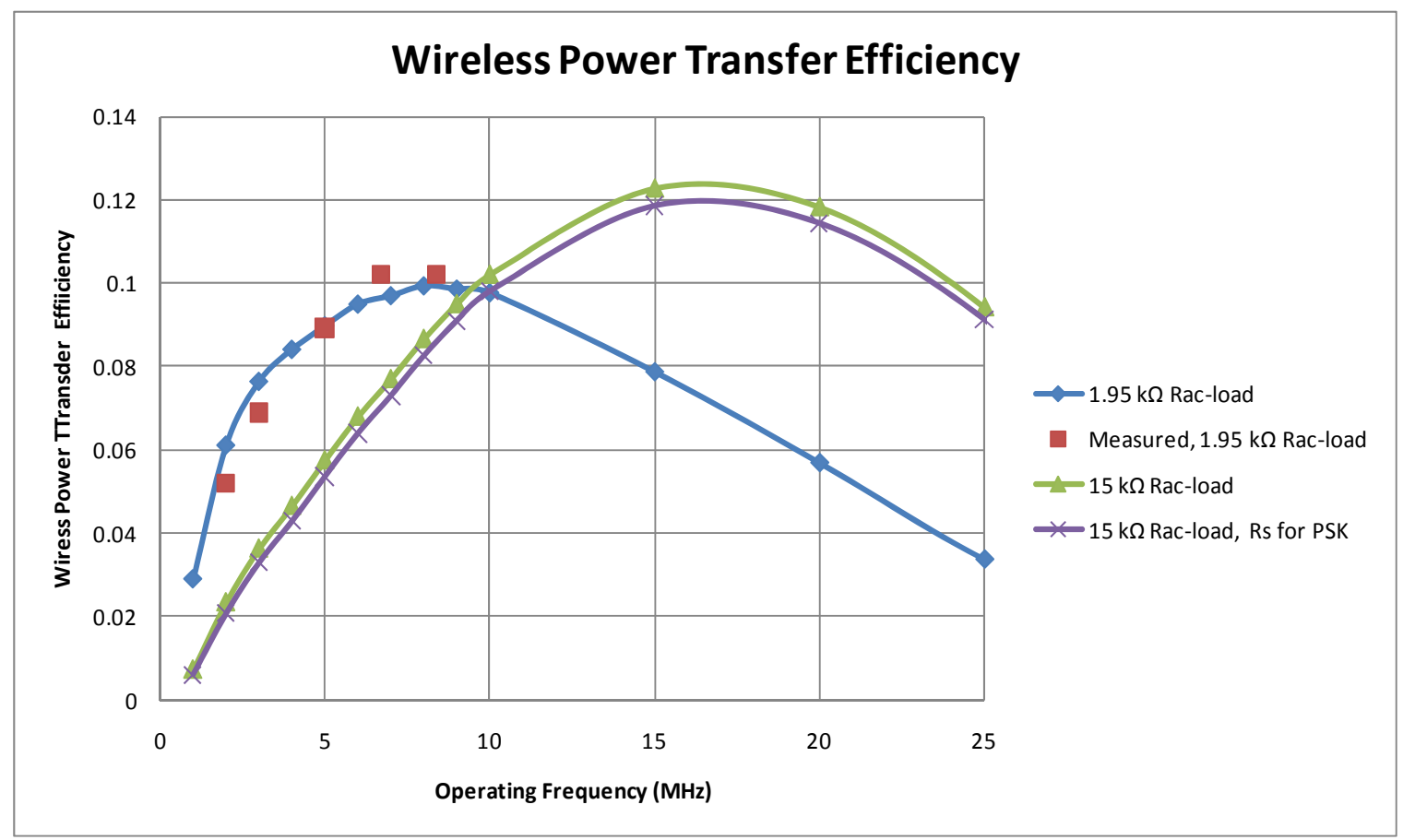

Figure 6-12. Wireless Power Transfer Efficiency $\left(R_{A C-L o a d}=15 \mathrm{k} \Omega\right)$

Once PSK modulation is enabled on the integrated circuit, as described in Section 5.5, the wireless power transfer efficiency is reduced by about 55\% to approximately $3.8 \%$.

The total power delivered by the secondary tank is about $(3.25 \mathrm{~V})^{2} /(2 \times 15 \mathrm{k} \Omega)=$ $350 \mu \mathrm{W}$. Figure 6-13 shows a pie chart detailing the breakdown of power consumption in the implantable microsystem. It can be seen that over half of the system power is consumed by the linear regulators and the voltage doubler circuit. The front-end amplifier only consumes about $4 \%$ of the total system power. 


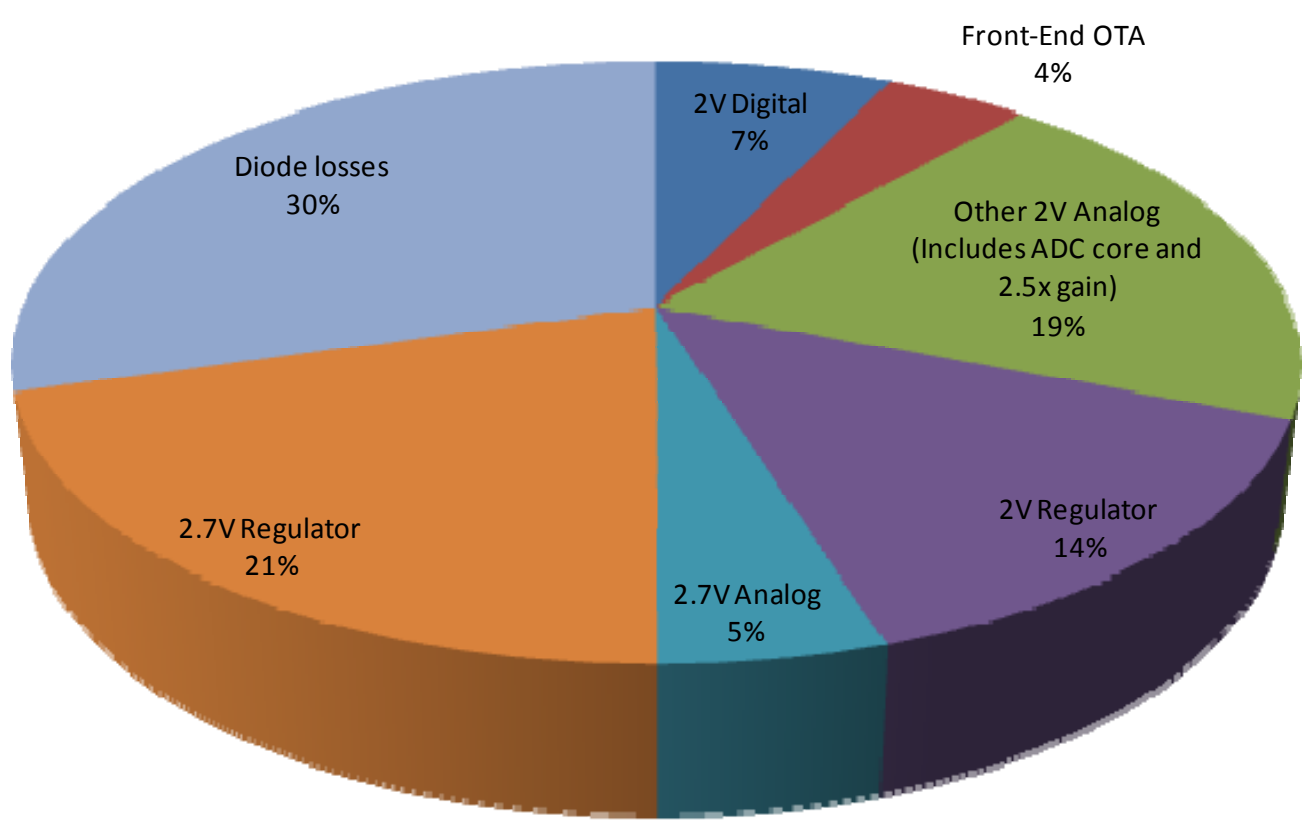

Figure 6-13. EMG Microsystem Power Consumption Breakdown

The Class-E powering system is simulated and measured to have approximately $8.25 \%$ power transfer efficiency as measured from the Class-E DC power supply $\left(\mathrm{V}_{\mathrm{DD}} \mathrm{x}\right.$ $\left.\mathrm{I}_{\mathrm{DD}}\right)$ to the total power delivered to $\mathrm{R}_{\mathrm{AC}-\mathrm{load}}$ at $1 \mathrm{~cm}$ coils separation distance with ideal tuning of the secondary tank. This measurement is conducted with a total series resistance in the primary tank, $\mathrm{R}_{\mathrm{s}}$, equal to $2.5 \Omega$ (to set the tank Q properly for PSK data telemetry bandwidth requirement). In this configuration, a 30-turn implantable secondary coil is inductively coupled to a 10-turn spiral primary coil, operating at $8 \mathrm{MHz}$. The power required by the Class-E amplifier can be calculated from the power consumption of the secondary circuit, as shown in Equation 6-13.

$$
P_{\text {Class }-E}=\frac{P_{\text {Secondary }}}{\eta_{\text {eff }}}=\frac{350 \mu \mathrm{W}}{8.25 \%}=4.24 \mathrm{~mW}
$$

The Class-E amplifier takes in a constant current from $V_{D D}$ through the RF choke inductor, so it can be modeled as a DC load. Based on the Class-E design equations, simulations, and measurements, the input resistance, $R_{D C_{-} \text {Class-E}}$, is approximately $4.4 \Omega$. 
The minimum required DC supply voltage for the Class-E amplifier to deliver enough power to the secondary tank can be calculated as Equation 6-14.

$$
P_{\text {Class }-E}=\frac{V_{D D}^{2}}{R_{D C, \text { Class }-E}}=4.24 \mathrm{~mW} \rightarrow V_{D D}=\sqrt{4.4 \Omega \cdot 4.24 \mathrm{~mW}}=137 \mathrm{mV}
$$

The RF voltage gain for a linear system shown in Figure 5-1 at $8 \mathrm{MHz}$ with Rs of $2.5 \Omega$ is shown in Equation 6-15.

$$
A_{V}=\frac{V_{a c_{-} \text {out }}}{V_{a c_{-} \text {in }}}=\frac{\omega^{2} M L_{2}}{(\omega M)^{2}+R_{S} R_{2}+R_{S} \frac{\left(\omega L_{2}\right)^{2}}{R_{\text {ac_load }}}}=22.3
$$

$\mathrm{V}_{\text {in-ac }}$ can be defined as the peak AC voltage, obtained by multiplying the peak AC current by Rs, as shown in Equation 6-16.

$$
V_{\text {in-ac }}=I_{A C-\text { Peak }} R_{S}=\frac{\sqrt{\pi^{2}+4}}{2} \frac{V_{D D}}{\left(\frac{\pi^{2}+4}{8} R_{S}\right)} R_{S}=\frac{4}{\sqrt{\pi^{2}+4}} V_{D D}=1.074 V_{D D}
$$

A "Class-E voltage gain" from the Class-E DC supply voltage to the peak-to-peak AC voltage across the secondary tank can be defined in Equation 6-17. This computed value of $\mathrm{A}_{\mathrm{V}, \text { Class-E }}=22.1$ compares favorably with the classical Class-E voltage gain,

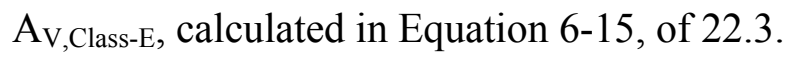

$$
\begin{aligned}
& \frac{V_{\text {out }-a c}}{V_{D D}}=\sqrt{\frac{2 R_{\text {ac-load }} \eta_{\text {eff }}}{R_{D C}}}=\sqrt{\frac{2 \cdot 15 \mathrm{k} \Omega \cdot 0.0826}{4.4 \Omega}}=23.73 \\
& \rightarrow A_{V, \text { Class }-E}=\frac{V_{\text {out }-a c}}{V_{\text {in }-a c}}=\frac{23.73}{1.074}=22.1
\end{aligned}
$$

Since there is a "large" gain from $\mathrm{V}_{\mathrm{DD}}$ to the peak-to-peak voltage across the secondary tank, care must be taken to not provide too much supply voltage to the Class-E amplifier so that a large voltage is not generated across the implantable tank. 
After PSK is enabled, the wireless power transfer efficiency of the system is reduced to approximately $3.8 \%$. This requires additional power to be delivered by the Class-E amplifier during full operation of the chip as shown in Equation 6-18.

$$
P_{\text {Class }-E}=\frac{P_{\text {Secondary }}}{\eta_{\text {eff }}}=\frac{350 \mu \mathrm{W}}{3.8 \%}=9.2 \mathrm{~mW}
$$

Once wireless RF powering using the Class-E amplifier was successfully demonstrated, initial verification of the passive digital PSK data telemetry link was performed. In Figure 6-14, sample digital PSK data is transmitted from the RF-powered ASIC and received over the $1-\mathrm{cm}$ inductive data telemetry link by a discrete digital phase recovery circuit as shown previously in Figure 3-7. The top trace is the transmitted data recorded at the PSK switch control line, and the bottom trace is the received data recorded at the output of the PSK demodulator.

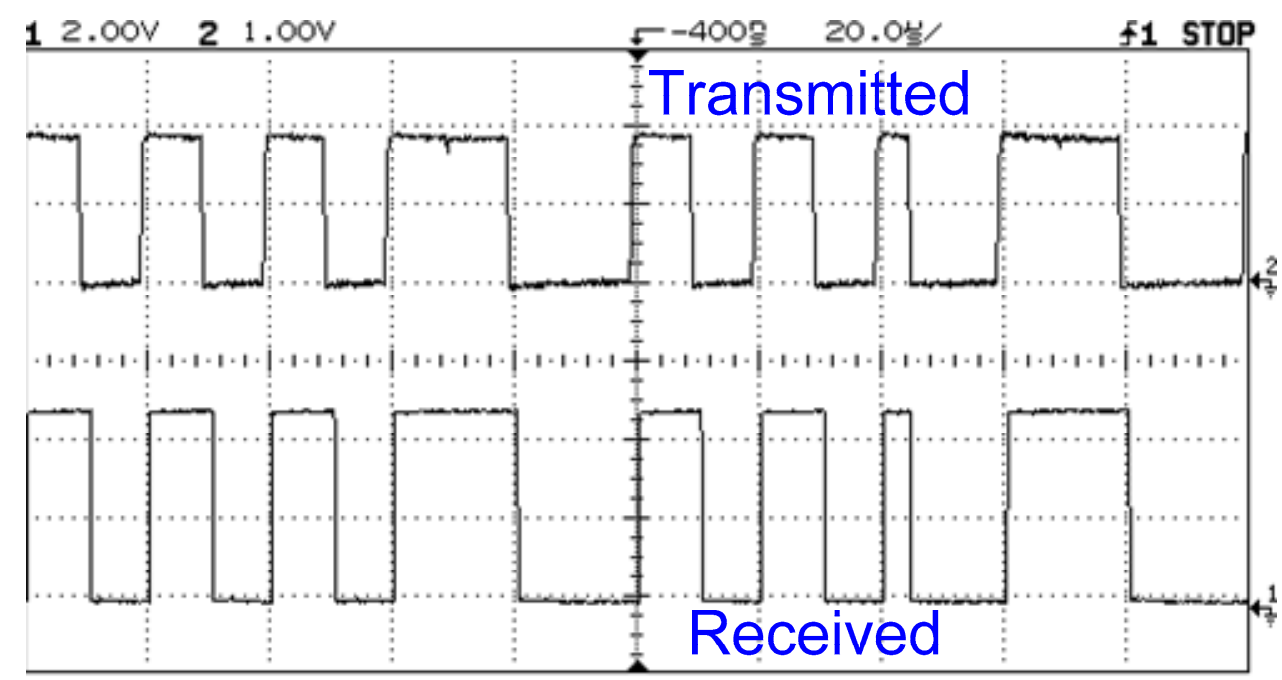

Figure 6-14. Transmitted and Received PSK Data

Once the data link was operational, an extended test was performed to estimate the bit error rate (BER). With 1 to $2 \mathrm{~cm} \mathrm{RF}$ coil separation and good alignment, there were typically about 50,000 data samples before a parity error was detected, where each 
sample consists of 24 data bits. This corresponds to a bit error rate as shown in Equation 6-19.

$$
B E R=\frac{1 \text { parity error }}{50,000 \text { samples }} \cdot \frac{1 \text { sample }}{24 \text { bits }}=8 \times 10^{-7}
$$

At 2,000 samples per second, that BER corresponds to 1 rejected sample every 25 seconds. With a good alignment and small coils spacing with the coils almost touching, an improved BER on the order of $1 \times 10^{-7}$ or better was observed with the system sometimes running for several minutes with no parity errors. With a poor alignment (but still good enough to power up and stream data), typically around 10,000 samples were received before parity error occurred, corresponding to a BER of $4 \times 10^{-6}$.

\subsection{In Vivo Animal Test Results}

The wireless EMG sensing system was tested in vivo by implanting intramuscular electrodes into a rat's hind limb. The small muscle size in the rat hind limb precluded the use of epimysial electrodes in this test. Intramuscular electrodes also provide an EMG signal similar to that of epimysial electrodes. They consist of a plastic barb to secure the

electrodes in the muscle site and two recording electrode sites formed by wrapping stainless steel wire around the cable. The two leads are wound helically and encapsulated in medical grade silicone. The intramuscular electrodes were fabricated at Ardiem Medical, Inc. and are depicted in Figure 6-15. 


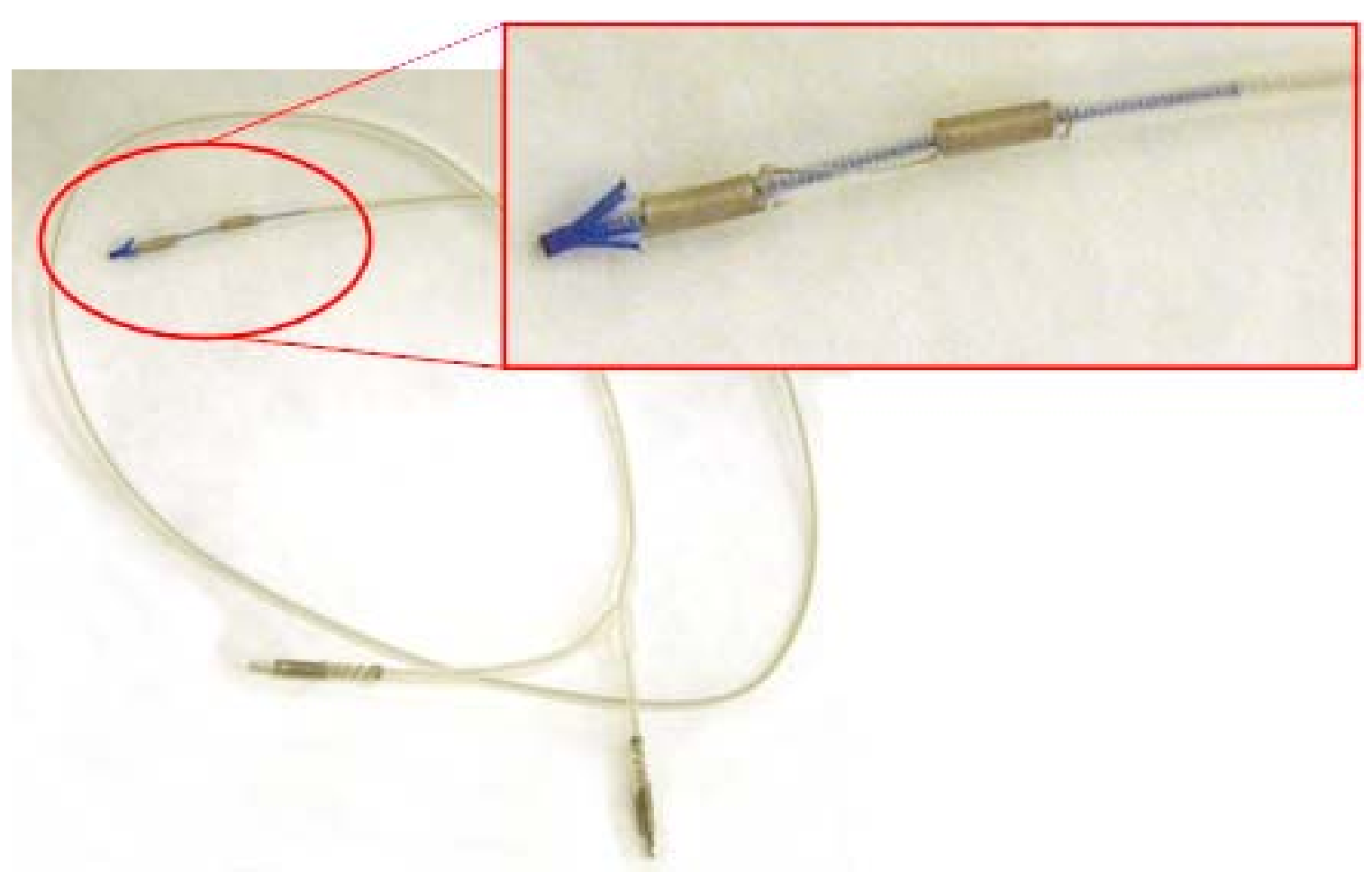

Figure 6-15. Intramuscular EMG Electrodes

The electrodes were inserted into an opening in the skin behind the rat's skull and fed under the skin on the rat's back. The electrodes were accessed through an incision above the rat's hind limb and inserted into the muscle of the hind limb. The skin was sutured closed and the electrode lead terminals were cemented into a cap mounted on top of the rat's skull. An overview of the surgery with a close-up view of the insertion of the intramuscular electrodes in the rat hind limb is presented in Figure 6-16. 

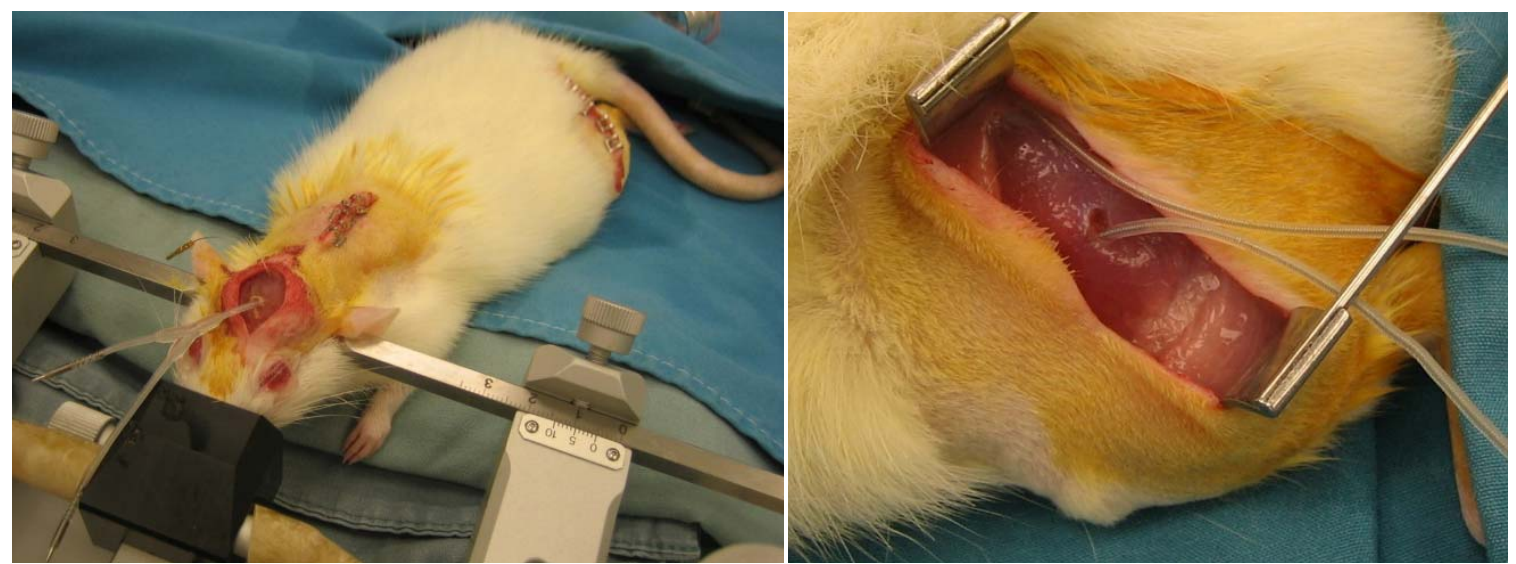

Figure 6-16. Rat in Surgery

The rat recovered fully after the surgery. A twisted pair of wires was attached to the implanted electrode leads at the rat's skull cap as depicted in Figure 6-17 to connect the implanted electrodes to the EMG interface electronics. Twisted wires were used to minimize differential or unbalanced coupling of $60 \mathrm{~Hz}$ and other interference signals. During the test, the rat was awake and not sedated.

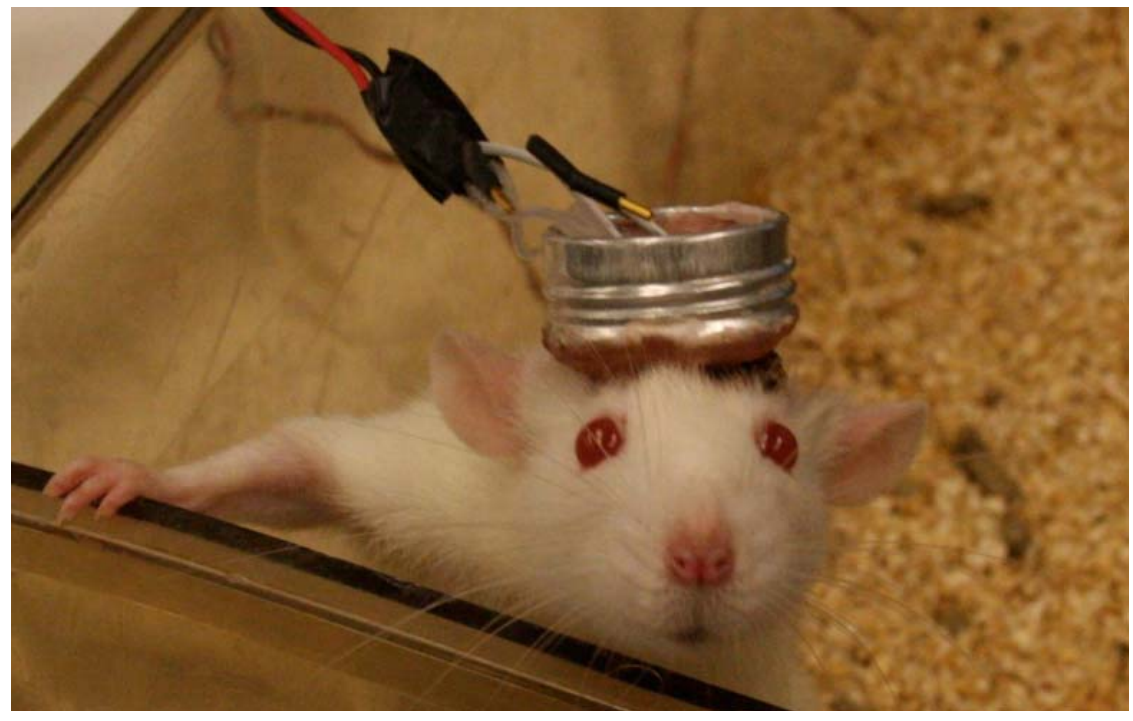

Figure 6-17. Rat with Skull Cap

The rat test setup is shown in Figure 6-18. The electronic interface, which was not implanted in the rat, was located in a shielded box nearby and connected to the EMG electrodes through approximately 1 meter twisted pair wires. The electronics are 
powered wirelessly via near field inductive coupling. The Class-E amplifier and PSK telemetry transceiver are powered by two power supplies: a $5 \mathrm{~V}$ supply for the oscillator and PSK demodulation blocks and an adjustable power supply to power the Class-E amplifier. The received PSK data stream is sampled by a PC with a National Instruments USB data acquisition board. The EMG data contained within the digital PSK stream is decoded, visualized, and logged to file using custom LabVIEW interface software.

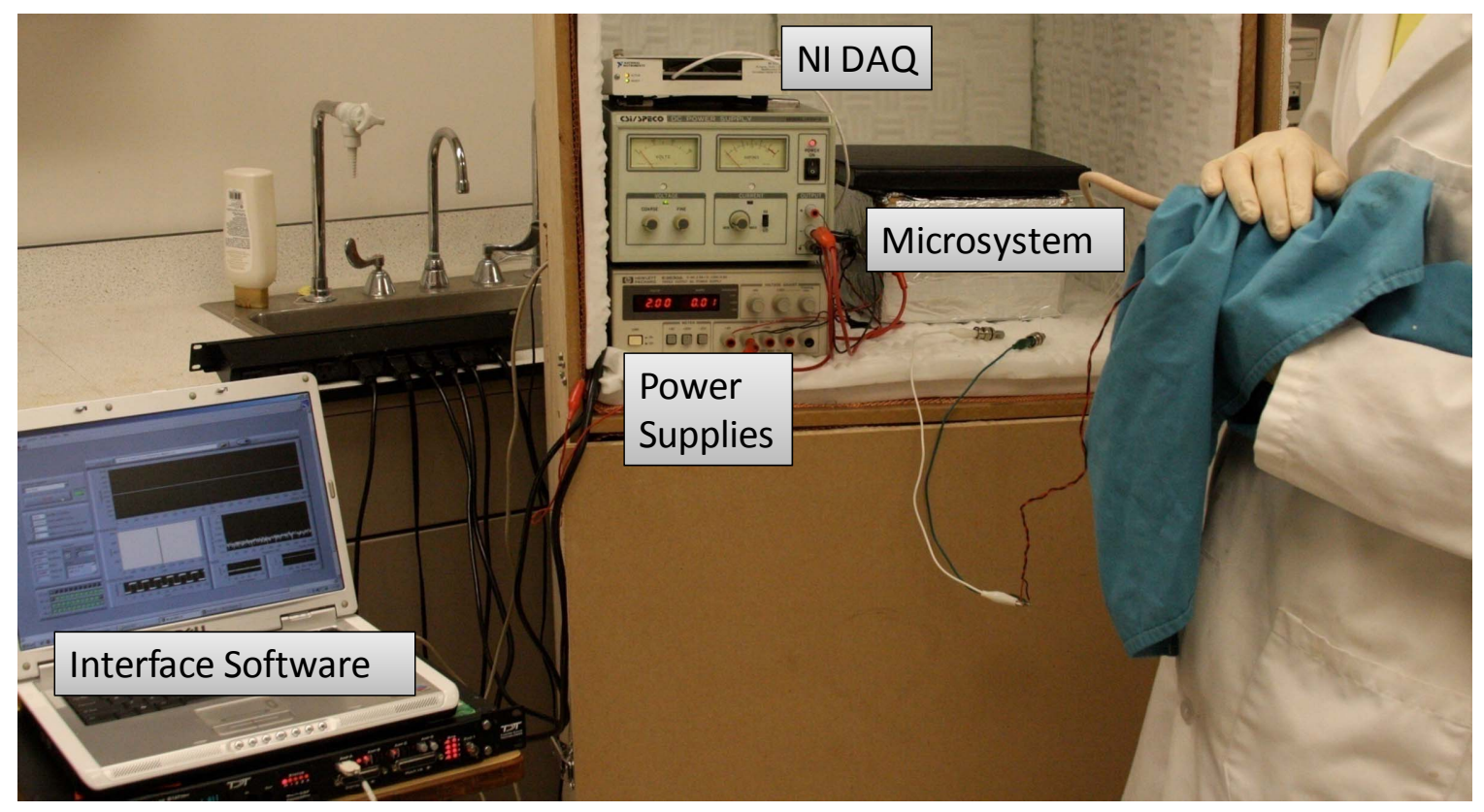

Figure 6-18. Rat Test Setup

The entire system is shown in Figure 6-19. The system consists of the integrated EMG interface electronics, the wireless power and data transceiver, and an external logic circuit to correct for a hardware flaw ASIC design. 


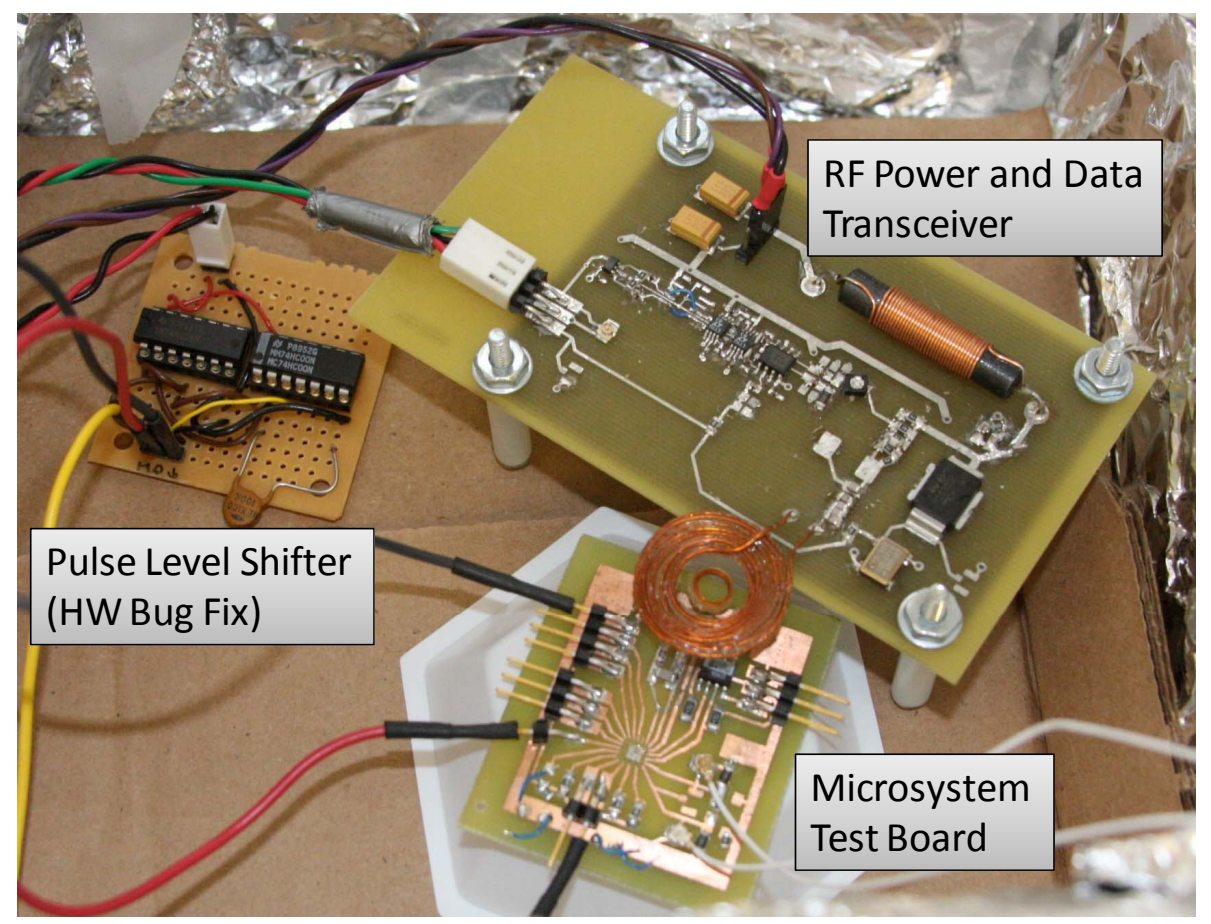

Figure 6-19. Wireless Microsystem Setup

Data were first collected with a pair 1-meter-long wires attached to OTA differential input. These wires were twisted together and electrically shorted together at the far end. This setup was used to characterize a baseline system performance in the test environment, and also to verify that $60 \mathrm{~Hz}$ coupling into the long wires would not be a significant problem, before attempting to measure in vivo EMG signals from the rat. The system was running in wireless powering and data telemetry mode with real-time data monitoring and logging using custom LabVIEW interface software. Figure 6-20 shows time domain and voltage power spectral density plots of data collected during this test. The data are represented as digital codes from 0 to 2047 corresponding to differential input signals from $-5 \mathrm{mV}$ to $+5 \mathrm{mV}$ with a mid-code value of 1023 corresponding to a $0 \mathrm{~V}$ differential input signal. The OTA input-referred RMS noise measured with this long wire test is $6.6 \mu \mathrm{V}_{\mathrm{RMS}}$ within $1 \mathrm{kHz}$ bandwidth, equivalent to 1.26 ADC LSBs. 

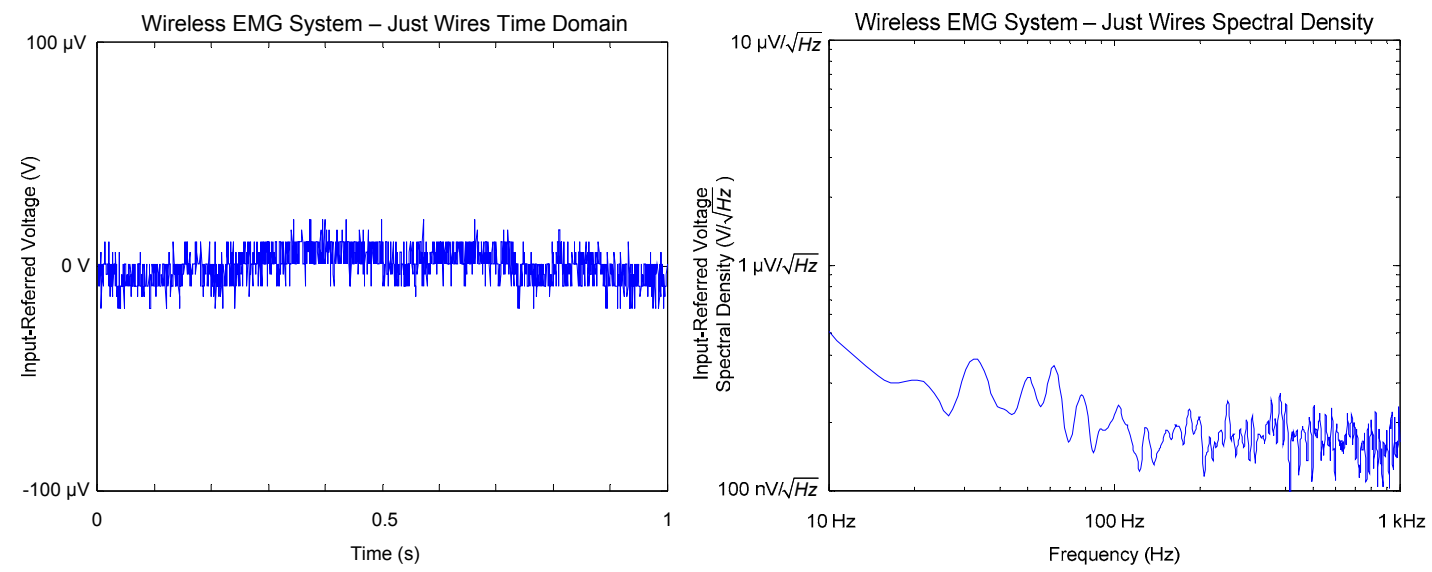

Figure 6-20. Measurement with Shorted Long Wires

Next, the long interface wires were attached to the differential intramuscular EMG electrode leads that were implanted into the rat's hind limb. Data were recorded with the rat at rest with minimal EMG activity to show the background biological activity level. These data are shown in Figure 6-21. The input-referred integrated background signal is $19.6 \mu \mathrm{V}_{\mathrm{RMS}}$, equivalent to 3.7 ADC LSBs. Also, the signal shows band-limited behavior with a low-pass cutoff at approximately $250 \mathrm{~Hz}$.
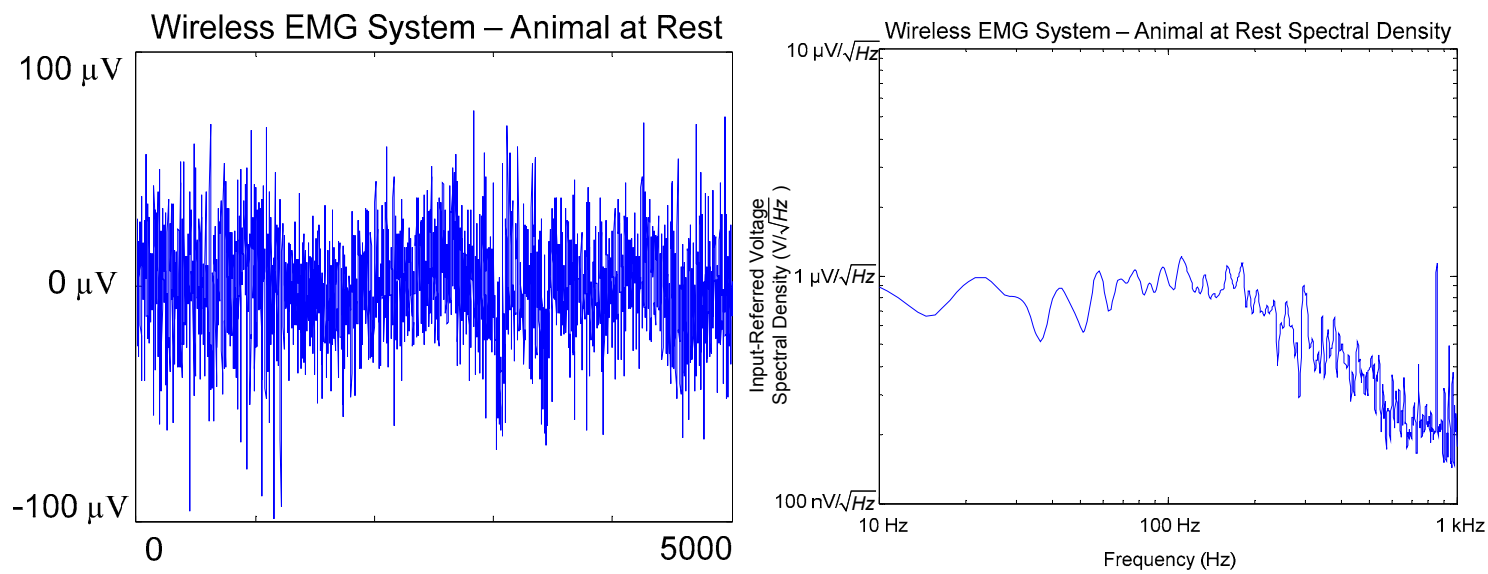

Figure 6-21. Long Wires Connected to Intramuscular Electrodes, with Resting Animal

The rat experimental set-up is shown in Figure 6-22. Light pulling force was applied to the rat's hind limb in which the intramuscular EMG electrodes are implanted, causing it to extend. The rat responded by contracting the muscles in its leg to counteract 
the pulling force. This muscle contraction is recorded by the EMG sensing microsystem simultaneously with a Tucker-Davis RA16PA battery-powered, optically isolated preamplifier connected to a RX5 Pentusa Base Station for baseline comparison to the wireless microsystem. In each pair of plots, a zoomed-out plot of a longer time scale is paired with a zoomed-in plot showing the commercial system data overlaid with the wireless microsystem data. Both data sets are high-pass filtered at $1 \mathrm{~Hz}$ to reduce effects of cable motion artifacts.
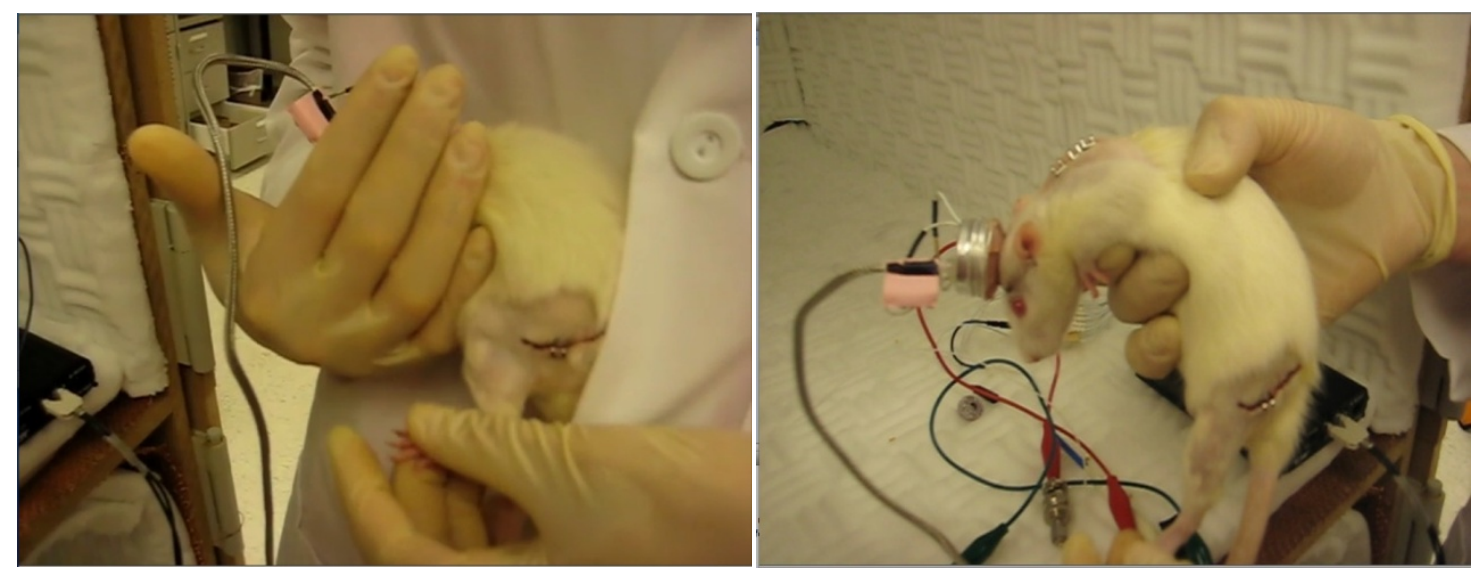

Figure 6-22. Rat Test Experiment

Figure 6-23 shows recorded data from three rat hind limb contractions spaced about one second apart. In this trial, the rat's hind limb was pulled into extension, and the rat responded by pulling it back. In each successive trial, a reduced force was applied to the leg. The diminishing response is clearly visible in the figure. The plot on the right is a zoomed-in version of the first pulse, showing approximately $80 \%$ correlation between the data recorded by the implantable EMG system and the commercial system over the duration of the pulse. The peak-to-peak differential amplitude of $8 \mathrm{mV}_{\mathrm{PP}}$ seen in the largest pulse closely matches the expected maximum signal level of $10 \mathrm{mV}_{\mathrm{PP}}$. 

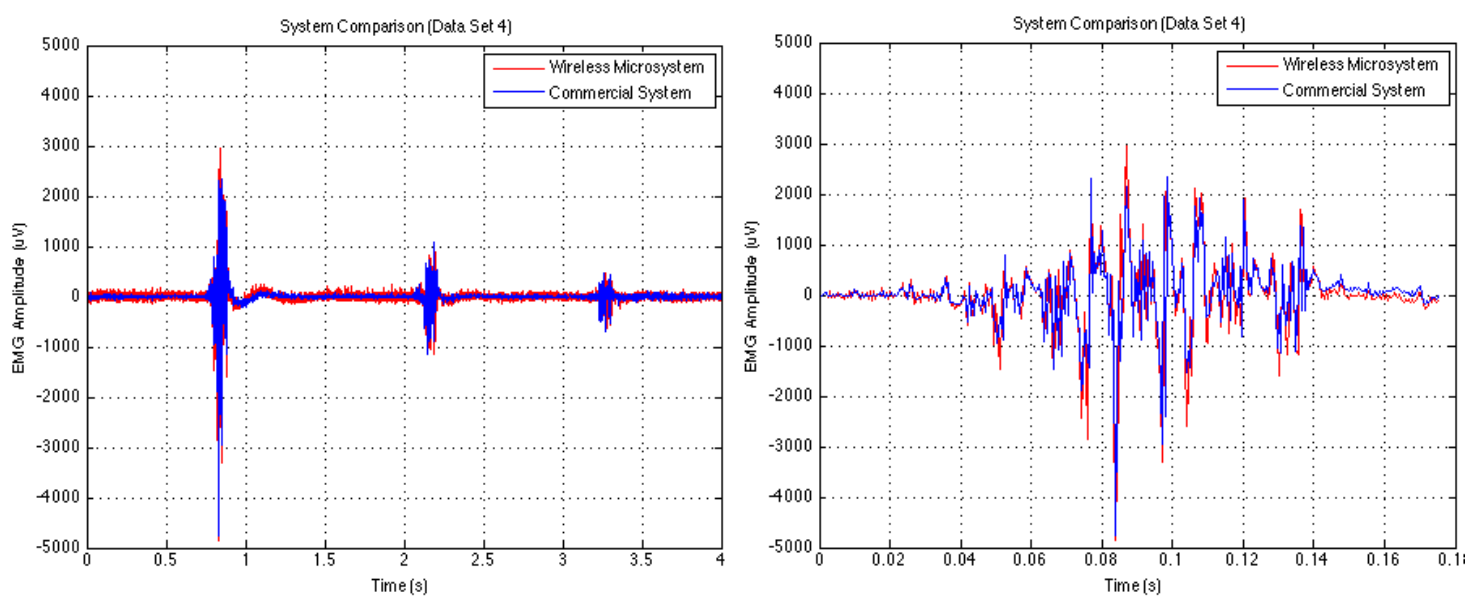

Figure 6-23. Three Rat Contractions in Four Seconds

In Figure 6-24, a sustained extension force was applied to the rat's leg and it responded by pulling it back quickly with three short bursts within one second. The zoomed-in section on the right is a close-up of the first pulse.
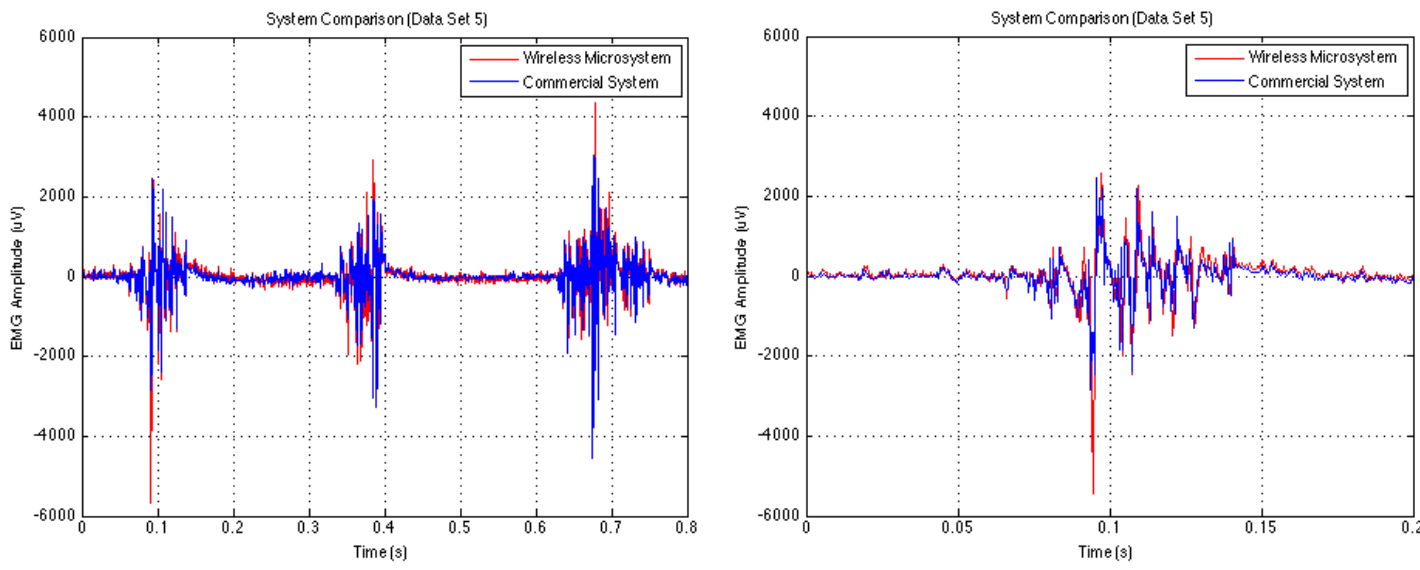

Figure 6-24. Three Quick Rat Hind Limb Contractions

In Figure 6-25, the rat responded to the leg extension by contracting the leg muscles in two quick bursts, waiting about two seconds, and then making another two quick contractions. The zoomed-in data are from the first two contractions. 

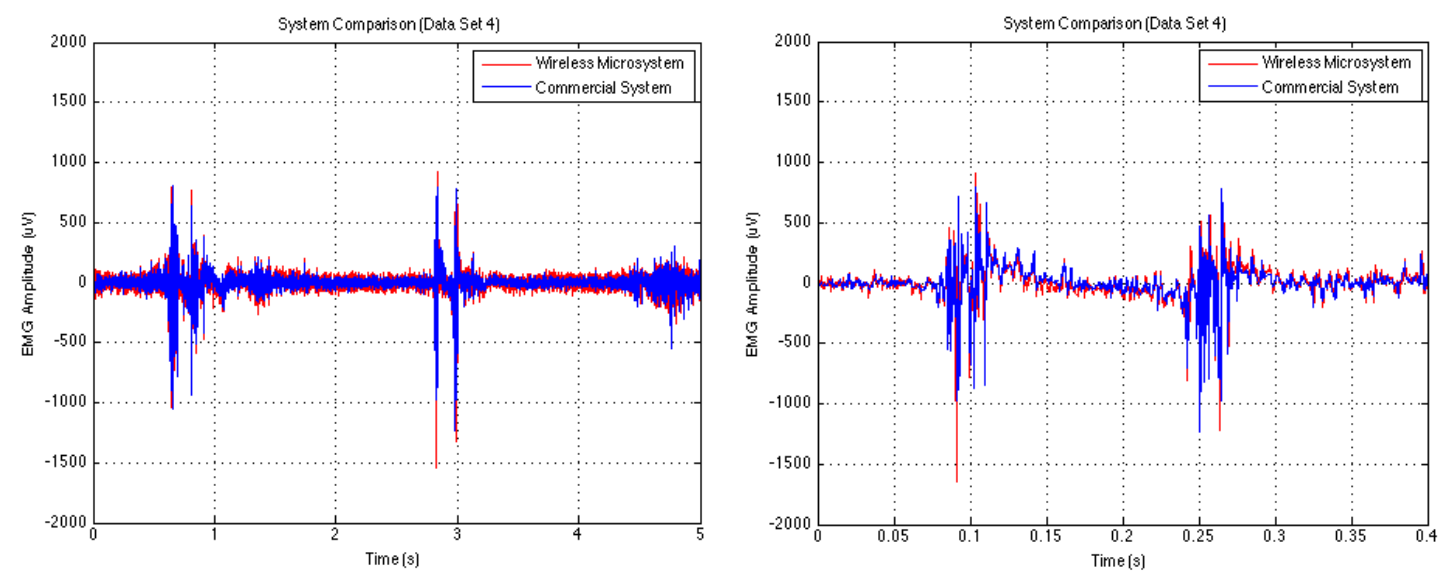

Figure 6-25. Two Sets of Two Quick Rat Leg Contractions

In order to compare the two data sets, a MATLAB routine was developed to automatically align the data by resampling and shifting the data until correlation is maximized. The correlation coefficient for periods of data that have EMG activity from 60 samples at $2 \mathrm{kHz}$ to 2000 samples at $2 \mathrm{kHz}$ was measured. The effect of performing a low pass filter on both data sets and recomputing the correlation coefficient was also investigated. Since most of the EMG signal power is typically contained within a $250 \mathrm{~Hz}$ bandwidth, it is hypothesized that much of the recorded data above $500 \mathrm{~Hz}$ will be uncorrelated system noise or electronic interference. It is evident from data shown in Figure 6-26 that the correlation between the two systems does increase linearly with decreasing cutoff filter frequency. The maximum found correlation coefficients are higher for smaller data windows. This is because the correlation is highest between the two systems during high-SNR periods of the data, such as during strong muscle contraction. As the data windows are expanded to include more low-SNR data, such as during periods of muscle inactivity, the maximum found correlation coefficient decreases. For large correlation windows, the maximum found correlation coefficient stays nearly constant. When comparing data within $500 \mathrm{~Hz}$ bandwidth and zooming in 
on an EMG pulse within 60 samples (30 ms), the correlation coefficient can be over 95\%. When using the full system bandwidth, the correlation can be over $80 \%$ during high-SNR sections of the data.

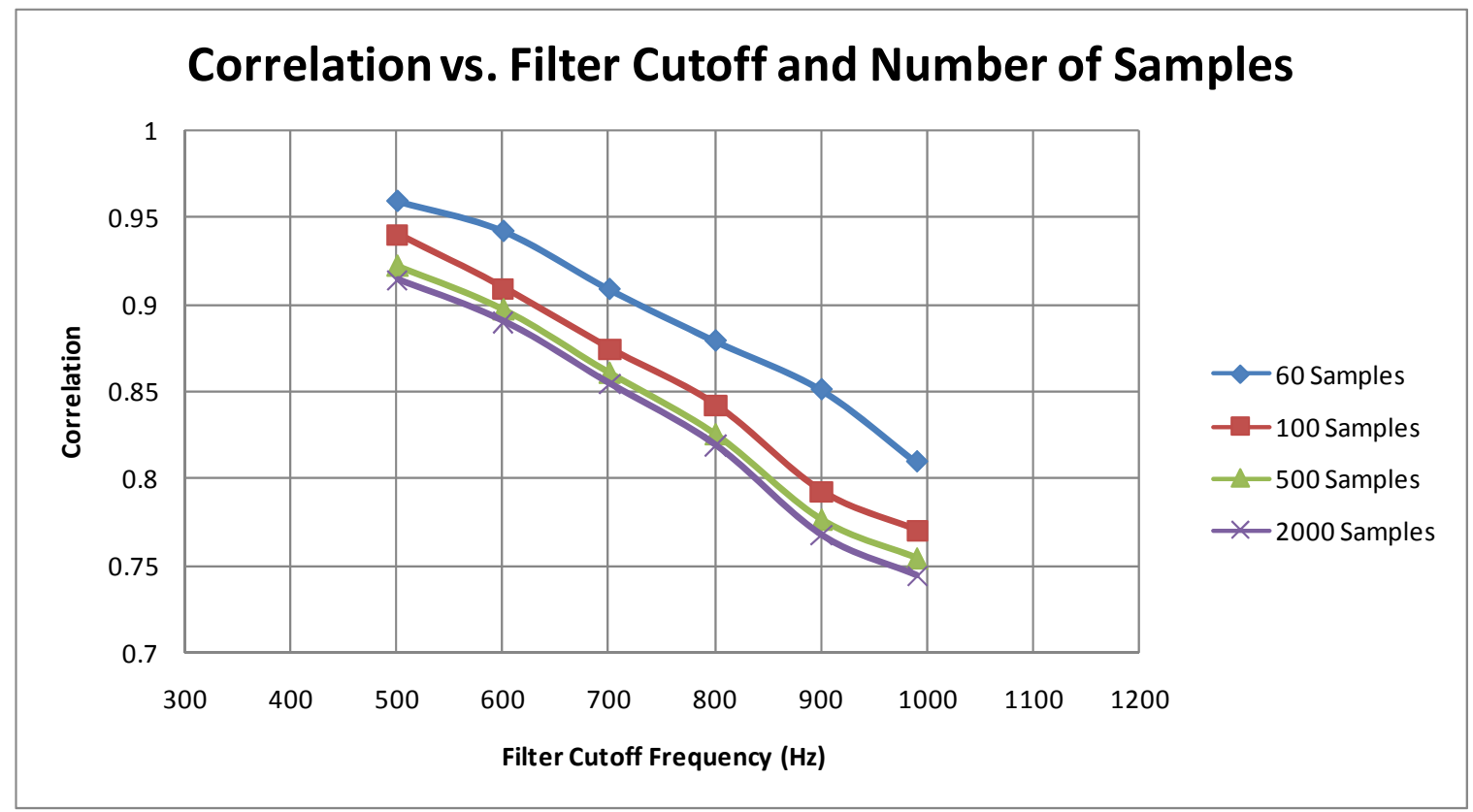

Figure 6-26. Correlation of Rat Test Data

\subsection{Summary}

In this chapter, electronic performance characterization of the wireless microsystem was presented. The OTA -3dB low-pass corner frequency measured to be $920 \mathrm{~Hz}$ and the high-pass corner frequency was measured to be $20 \mathrm{mHz}$. The measured OTA thermal noise floor was measured to be $78 \mathrm{nV} / \sqrt{\mathrm{Hz}}$ when referred to the OTA input. The $1 / f$ noise corner frequency is equal to $30 \mathrm{~Hz}$. The thermal noise is slightly higher than the originally designed value due to the reduced OTA bias current. Under battery power, the system achieves 9.4 ENOB, which matches the analytical calculations. Under RF power, additional tones in the ADC output spectrum and increased thermal noise floor reduce the 
system performance to $8.6 \mathrm{ENOB}$. The RF powering link was demonstrated to achieve a wireless power transfer efficiency of $8.25 \%$ with ideal tuning of the secondary tank. With PSK operation enabled, the wireless power transfer efficiency is reduced to $3.8 \%$ and the system achieves a typical data BER of $8 \times 10^{-7}$. The PSK system design could be modified in a future design to improve the power transfer efficiency without significantly degrading the bit error rate.

An in vivo evaluation was also performed in which intramuscular electrodes were implanted into a rat. For this test, the wireless microsystem was not implanted, but was tested in wireless powering and data operation mode outside the rat and interfaced with the implanted electrodes. Real-time data from the awake, moving rat were recorded simultaneously by a commercial biopotential recording system and the wireless microsystem. The data from both systems exhibited high correlation of over $80 \%$ when observing EMG pulses within the full system bandwidth and over $95 \%$ if the data are low-pass filtered to an EMG bandwidth of $500 \mathrm{~Hz}$. A background EMG signal of 19.6 $\mu \mathrm{V}_{\text {RMS }}$ was observed with the electrodes implanted in an at-rest rat. This background EMG signal is larger than the input-referred integrated system voltage noise and therefore sets the limit of the system resolution. The system demonstration of measuring a detailed EMG waveform from an awake rat is an excellent indicator that this system would work well to sense human EMG signals for prosthetic control. 


\section{Chapter 7}

\section{Conclusion and Future Work}

\subsection{Conclusion}

In this research, a wireless, implantable, battery-less EMG sensing microsystem has been developed. A custom ASIC consisting of a low-power, an EMG-sensing amplifier operated in moderate inversion, analog-to-digital conversion, RF-to-DC power rectification and regulation, and digital control logic was designed and fabricated in the AMI $1.5 \mu \mathrm{m}$ CMOS process occupying an area of approximately $2.2 \mathrm{~mm} \times 2.2 \mathrm{~mm}$. An $8 \mathrm{MHz}$ near-field RF inductive coupling link for wireless powering and passive digital PSK data telemetry has been optimized and fabricated. The wireless powering link achieves an $8.25 \%$ wireless power transfer efficiency at $1 \mathrm{~cm}$ separation with a tuned secondary tank. Received RF energy is converted to stable $2.1 \mathrm{~V}$ and $2.7 \mathrm{~V}$ DC supplies by on-chip voltage doubler circuitry and linear regulators. The differential, ac-coupled front-end electronics achieve an input-referred noise power spectral density of 78 $\mathrm{nV} / \sqrt{\mathrm{Hz}}$ and a closed-loop bandwidth of $920 \mathrm{~Hz}$ with DC baseline stabilization. The ASIC consumes $63.2 \mu \mathrm{A}$ from the $V_{\text {supply_low }}$ of $2.5 \mathrm{~V}$ and $19.8 \mu \mathrm{A}$ from $V_{\text {supply_high }}$ of 4.5 
V. The implantable system consumes a total of $350 \mu \mathrm{W}$ from RF powering, with $3.8 \%$ wireless power transfer efficiency during PSK operation. The system achieves 9.35 ENOB under battery power, corresponding to a minimum detectable EMG signal of 5.4 $\mu \mathrm{V}_{\mathrm{RMS}}$. Under wireless operation, the overall system achieves 8.6 ENOB, corresponding to a minimum detectable signal of $9 \mu \mathrm{V}_{\text {RMS }}$. An in vivo animal implant test in an awake rat shows the background EMG activity signal is approximately $20 \mu \mathrm{V}_{\mathrm{RMS}}$, thus limiting the system sensing resolution. The prototype microsystem achieves over $95 \%$ correlation with a commercial biopotential recording system within a $500 \mathrm{~Hz}$ EMG bandwidth, and achieves over $80 \%$ correlation when observing EMG activity over the full sensing bandwidth. The demonstrated performance is sufficient for measuring a high-quality EMG waveform for real-time intelligent powered prosthetic control.

\subsection{Future Work}

In order to achieve a fully-implantable EMG sensor, the custom ASIC, EMG interface electrodes, secondary coil, and off-chip capacitors must be integrated on a flexible, reliable substrate. Figure 7-1 shows an early prototype of this idea, which was developed at the Advanced Platform Technology Center. In this figure, the ASIC is bonded to a small but standard printed circuit board with its associated passives. This PCB carrier is then mounted to a thin and flexible liquid crystal polymer substrate that has been patterned with traces and EMG-sensing electrodes using conventional photolithography techniques. Laser vias are used to provide a contact through the substrate. In a future version, the ASIC may be bonded to an even smaller PCB or perhaps to the flexible substrate itself, covered in glob top epoxy resin, and encapsulated 
in medical-grade silicone. Flip-chip bonding the IC directly to the LCP substrate material has the potential to offer a reliable and robust connection. Another potential method to improve integration, minimize device size, reduce cost, and improve device-to-device consistency would be to move from a hand-wound secondary coil to a thick-film coil patterned on the LCP substrate surface. These techniques would require investigation into feasibility, reliability, and biocompatibility of the entire structure, as well as an analysis of the trade-off in terms of power transfer efficiency in employing a printed coil. As a scale reference, the IC is $2.2 \mathrm{~mm} \times 2.2 \mathrm{~mm}$ and the RF coil is about $1 \mathrm{~cm}$ diameter.

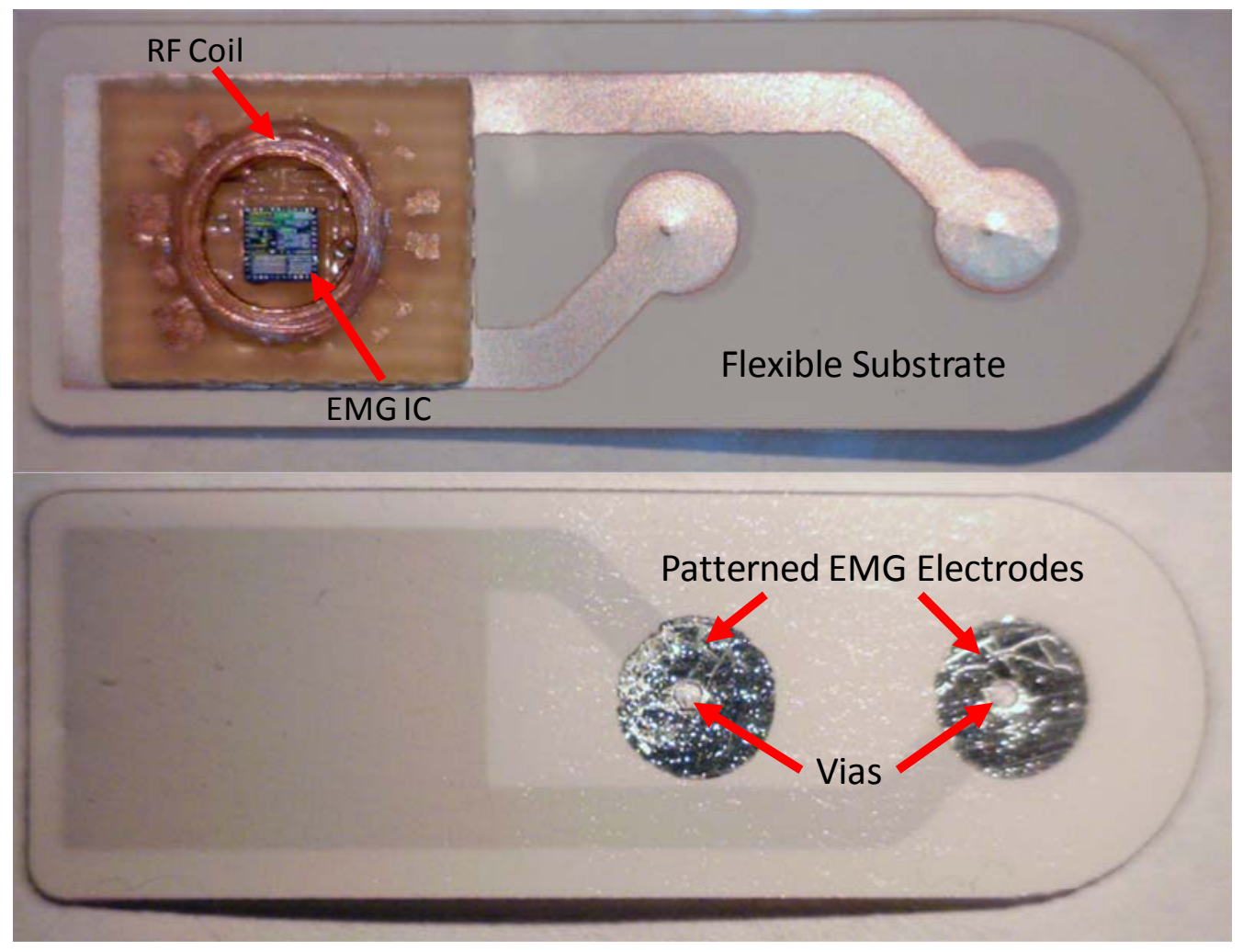

Figure 7-1. Mirofabricated EMG Electrode Prototype

Another example of a flexible EMG electrode package is shown in Figure 7-2. This device is based on the epimysial EMG electrode array originally designed at Case Western Reserve University and now manufactured by Ardiem Medical, Inc. The package is made of a flexible Dacron mesh encapsulated by medical grade silicone. The 
EMG sensing electrodes are made of thin platinum-iridium foils. The electrode leads are made of stainless steel and spot welded to the electrodes. In a future version of this package, the electrode leads would connect directly to the IC.

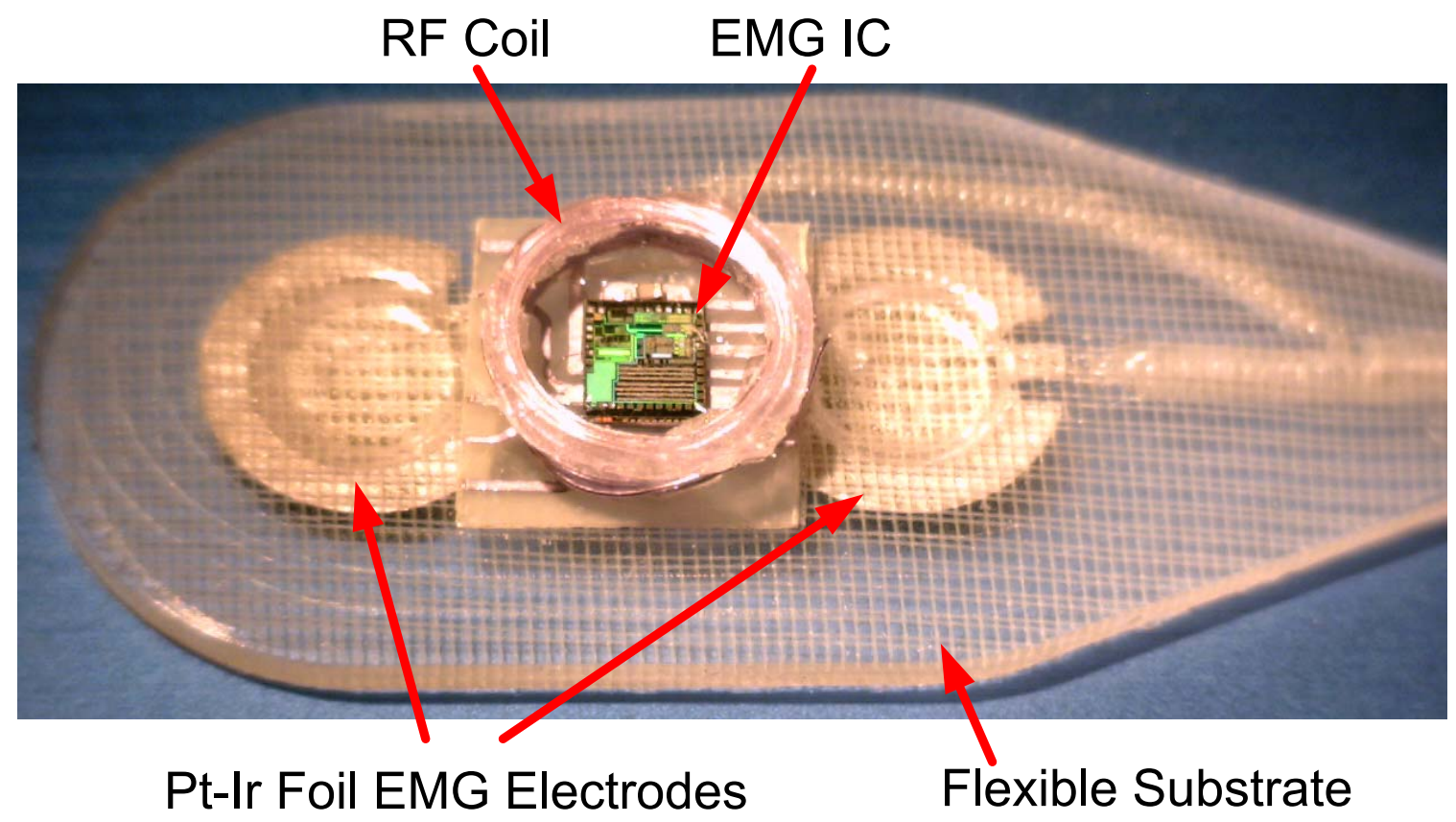

Figure 7-2. Ardiem flexible EMG prototype

To reduce the implantable EMG sensing system power consumption, it may be worthwhile to implement the voltage doubler with low voltage drop diodes. Even a small reduction in the voltage drop will have a dramatic effect on the total system power consumption. Implementing these as off-chip components would be a trade-off in terms of parts count, however. Effort may also be put in to improving the efficiency of the linear regulators, as these also consume a significant portion of the total system power. Also, if the system could be made to run off a single voltage supply, then the power of one linear regulator could be recovered and a full-wave bridge could be used for RF power rectification. 
Another possible path for future work in this project is to adapt the EMG sensing electronics and powering system to an Electroencephalograpic (EEG) or Electrocorticographic $(\mathrm{ECoG})$ recording device. EEG involves measuring lowfrequency brain-wave activity from the scalp surface or just below the scalp surface. EEG signal power is mostly contained below $40 \mathrm{~Hz}$ with signal levels of $10 \mu \mathrm{V}$ to 100 $\mu \mathrm{V}$ when measured from the scalp surface and $10 \mathrm{mV}$ when measured with sub-dermal electrodes. Figure 7-3 shows a 32-electrode EEG recording cap ${ }^{8}$.

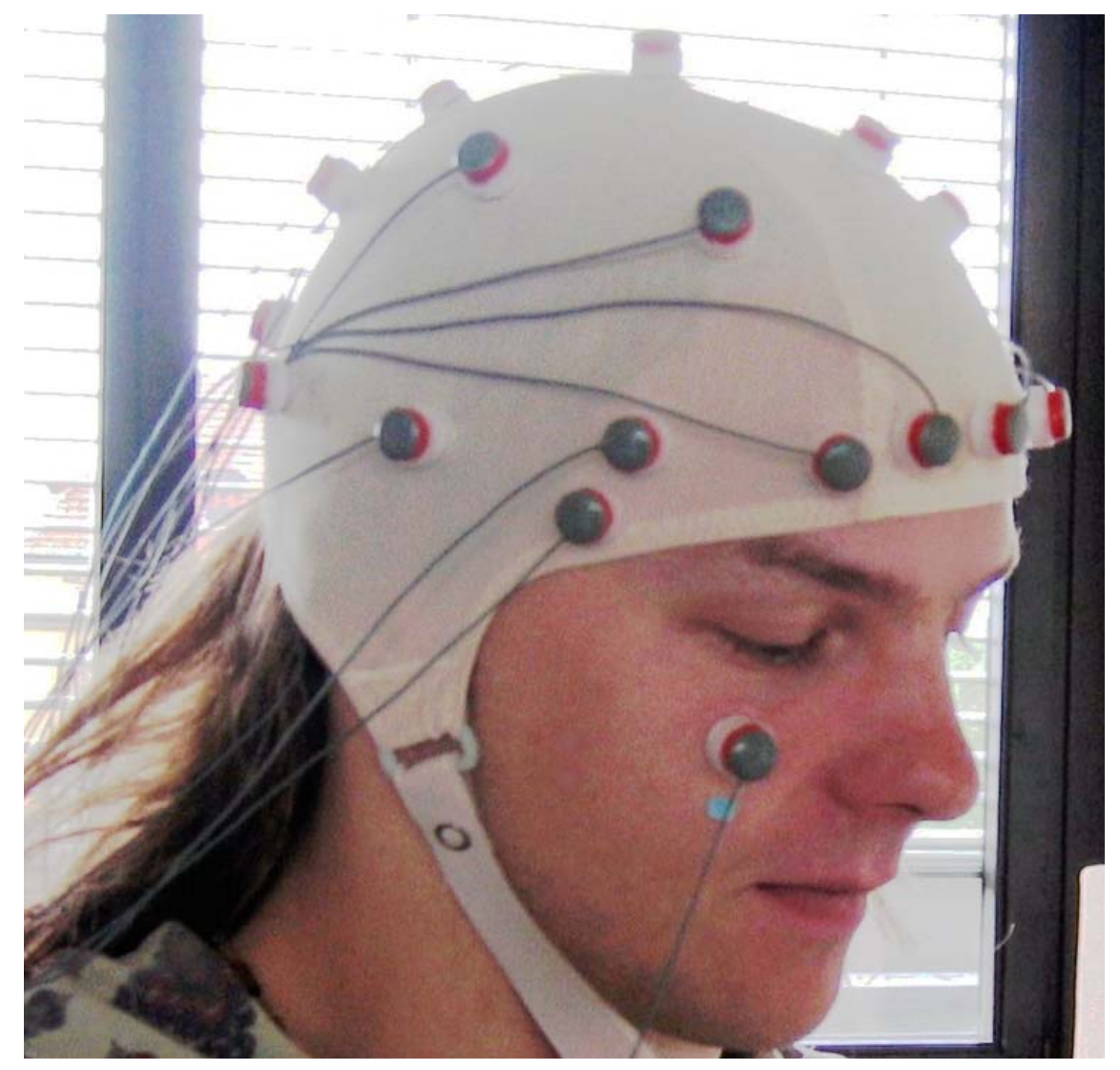

Figure 7-3. Surface EEG Recording

ECoG recording is one step more invasive than sub-dermal EEG, where electrodes are placed directly on the brain surface without breaching the blood-brain barrier, but requiring a portion of skull to be removed. ECoG is one step less invasive

\footnotetext{
${ }^{8}$ EEG with 32 electrodes, http://en.wikipedia.org/wiki/File:EEG_mit_32_Electroden.jpg
} 
than primary motor cortex electrode arrays such as the Michigan Probe or the UtahCyberkinetics Probe. ECoG signals have signal amplitudes up to $100 \mu \mathrm{V}$ and bandwidths up to $200 \mathrm{~Hz}$. They are sometimes preferred for long-term implant since they are less susceptible to EMG signal interference than EEG recordings.
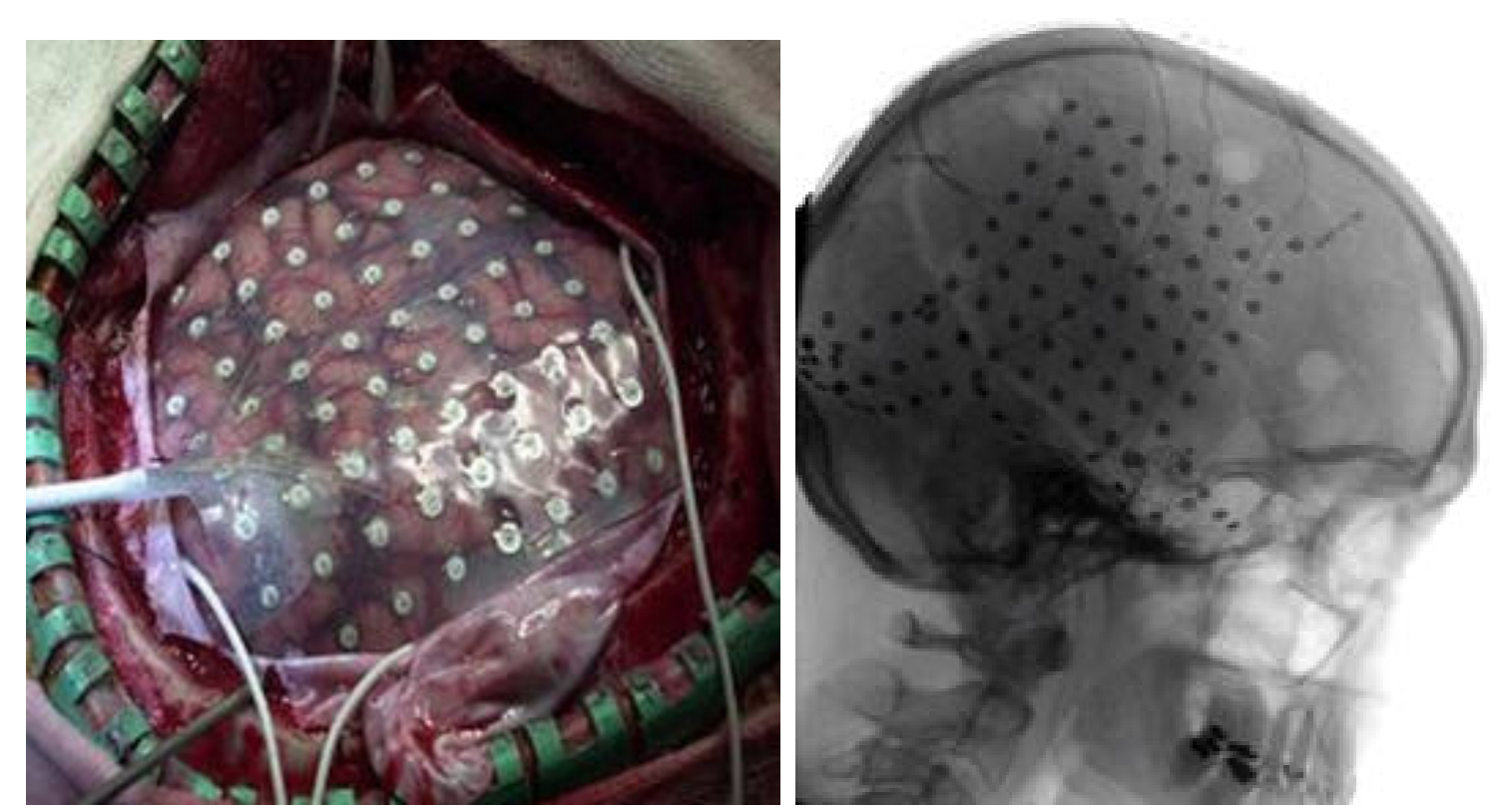

Figure 7-4. ECoG Implant and Post-Op X-Ray9

The EMG-sensing topology used in this project could be used to interface with either of these signal classes. The primary difference is that both EEG and ECoG recordings are made from an ensemble of many electrodes, where this project was designed to interface with one signal channel. So, if this project were to be adapted for EEG or ECoG, some trade-off would have to be considered between multiplexing the inputs or adding additional channels in parallel.

\footnotetext{
${ }^{9}$ Photo by Gerwin Schalk (Wadsworth Center, Albany, USA) and Kai Miller, Jeff Ojemann (University of Washington).
} 
Appendix I

\section{Correlation Alignment Algorithm}

In order to compare data sets that are sampled asynchronously and with potentially different sampling rates, it is necessary to resample and align those data sets before performing calculations such as the correlation (MATLAB function corrcoef). The MATLAB function below has been implemented to address this issue.

In general, given two data sets ( $\mathrm{y} 1$ and $\mathrm{y} 2)$ with best guess sampling rates ( $\mathrm{f} 1$ and $\mathrm{sf2}$ ), select a nearby target sampling rate fst and identify the base offset number and the data points to span the data of interest. The algorithm first resamples both data sets to the target frequency fst using the MATLAB resample function. The best guess for the original sampling frequency of $y 1$ is swept over a range of $+/-10 \mathrm{~Hz}$. For each trial of sampling frequency, the two data sets are aligned over an offset range of 100 data points. For each alignment trial, the correlation coefficient is calculated and recorded (using corrcoef). After the 100 point alignment sweep, the maximum correlation value and its corresponding sweep index are saved. This is repeated for each resampling trial. After the 20 resampling trials, find the trial (best guess of sampling 
frequency) which had the best overall correlation, and also the offset that yielded this maximum correlation. These results are sflout, which is the best guess of sampling frequency of $y 1$, offset, which is the alignment offset, and corr, which is the best recorded correlation coefficient.

Finally these parameters are reapplied to the incoming data to create aligned, resampled output data y1out and y2out.

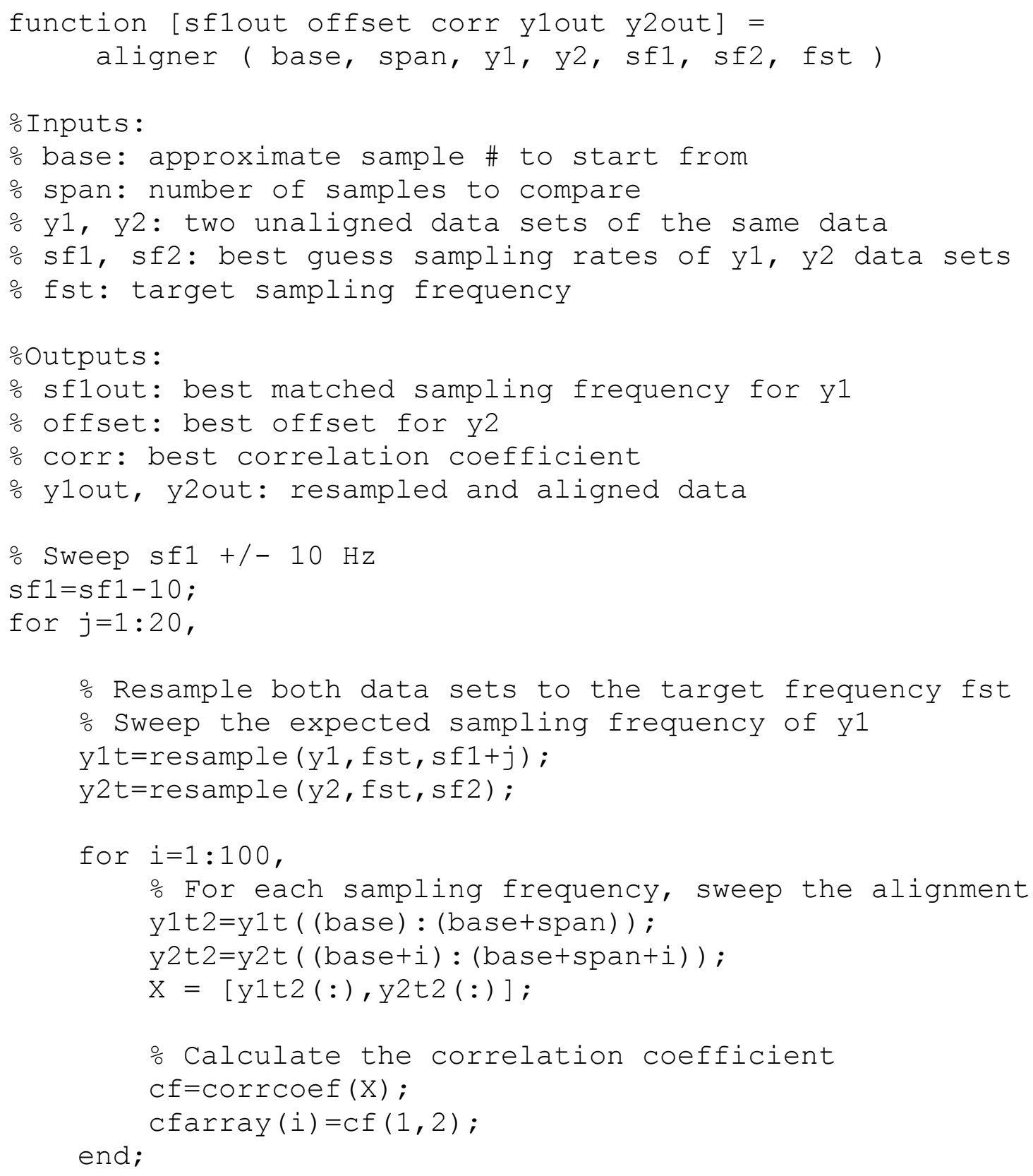


\% Find the best correlation from each frequency $[c f m a x(j)$, cfmaxindex $(j)]$ =max (cfarray);

end;

\% Find the best correlation overall

[corr, maxcorrindex] = $\max (\mathrm{cfmax})$;

\% Best matched sampling frequency for y 1

sflout = sfl+maxcorrindex;

$\div$ Best offset for y2

offset $=$ cfmaxindex (maxcorrindex);

\% Resample data with best parameters

ylt $=$ resample (y1, fst, sflout) ;

y2t $=$ resample (y2, fst, sf2);

ylout=y1t ( (base): (base+span));

y2out $=y 2 t($ (base+offset) : (base+span+offset) ); 


\section{References}

[1] G. DeMichele, P. R. Troyk, D. Kerns, and R. F. Weir, "An Implantable Myoelectric Sensor Based Prosthesis Control System," in Proceedings of the 28th IEEE EMBS Annual International Conference, Aug. 2006, pp. 2970-2973.

[2] G. E. Loeb, R. A. Peck, W. H. Moore, and K. Hood, "BION system for distributed neural prosthetic interfaces," Medical Engineering \& Physics, vol. 23, pp. 9-18, 2001.

[3] P. R. a. D. G. A. Troyk, "Inductively-coupled Power and Data Link for Neural Protheses Using a Class-E Oscillator and FSK Modulation," in IEEE EMBS, 2003, pp. 3376-3379.

[4] R. R. Harrison and C. Charles, "A Low-Power Low-Noise CMOS Amplifier for Neural Recording Applications," IEEE Journal of Solid-State Circuits, vol. 38, no. 6, pp. 958-965, 2003.

[5] P. Cong, W. H. Ko, and D. J. Young, "Low Noise microWatt Interface Circuits for Wireless Implantable Real-Time Digital Blood Pressure Monitoring," in Custom Integrated Circuits Conference, San Jose, California, Sep. 2008, pp. 523-526.

[6] "IEEE Standard for Safety Levels with Respect to Human Exposure to RF Electromagnetic Fields," in IEEE/ANSI c95.1.

[7] N. O. Sokal, "Class-E RF Power Amplifiers," QEX, pp. 9-20, Jan. 2001.

[8] P. R. Gray, P. J. Hurst, S. H. Lewis, and R. G. Meyer, "Analysis and Design of 
Analog Integrated Circuits," in Section 3.5.3: The DC Transfer Characteristic of a Source-Coupled Pair. New York: John Wiley \& Sons, Inc., 2001, pp. 218-221.

[9] M. K. Kazimierczuk and D. Czarkowski, "Class E Zero-Voltage-Switching Resonant Inverter," in Resonant Power Converters. New York: John Wiley \& Sons, Inc., 1995, pp. 347-377.

[10] F. W. Grover, Inductance Calculations. New York: D. Van Nostrand Co., 1962.

[11] N. Chaimanonart and D. J. Young, "Remote RF Powering for Industrial Strain Sensing Micorsystem," IEEE Sensors Journal, vol. 6, no. 2, pp. 484-489, Apr. 2006.

[12] W. H. Cantrell, "Tuning Analysis for the High-Q Class-E Power Amplifier," IEEE Transactions on Microwave Theory and Techniques, vol. 48, pp. 2397-2402, Dec. 2000.

[13] M. Zimmerman, N. Chaimanonart, and D. J. Young, "In Vivo RF Powering for Advanced Biological Research," in The 28th Annual International Conference of the IEEE Engineering in Medicine and Biology Society (EMBS '06), New York City, Aug. 2006, pp. 2506-2509.

[14] N. Chaimanonart and D. Young, "A Wireless Batteryless In Vivo EKG and Core Body Temperature Sensing Microsystem with $60 \mathrm{~Hz}$ Suppression Technique for Untethered Genetically Engineered Mice Real-Time Monitoring," in Technical Digest of the 31 st Annual International Conference of the IEEE Engineering in Medicine and Biology Society, Minneapolis, MN, 2009, pp. 4872-4875.

[15] W. H. Ko, S. P. Liang, and C. D. F. Fung, "Design of radio-frequency powered coils for implant instruments," Medical and Biological Engineering and Computing, vol. 
15, pp. 634-640, Nov. 1977.

[16] D. J. Young, B. D. Farnsworth, and R. J. Triolo, "Wireless Implantable EMG Sensor for Powered Prosthesis Control," in The 9th International Conference on Solid-State and Integrated-Circuit Technology, Beijing, China, Oct. 2008, pp. 2541-2544.

[17] B. D. Farnsworth, R. J. Triolo, and D. J. Young, "Wireless Implantable EMG Sensing Microsystem," in IEEE Sensors Conference, Lecce, Italy, Oct. 2008, pp. $1245-1248$.

[18] B. D. Farnsworth, D. M. Taylor, R. J. Triolo, and D. J. Young, "Wireless In Vivo EMG Sensor for Intelligent Prosthetic Control," in The 15th International Conference on Solid-State Sensors, Actuators and Microsystems, Denver, Colorado, Jun. 2009, pp. 358-361.

[19] M. Suster, J. Guo, N. Chaimanonart, W. H. Ko, and D. J. Young, "Low-Noise CMOS integrated sensing electronics for capacitve MEMS strain sensors," in IEEE Custom Integrated Circuits Conference, Orlando, Florida, Oct. 2004, pp. 693-696. 